\title{
The Shielding Effectiveness of a Door and Frame Assembly
}

By

Rene Nyangezi, B.Eng

\begin{abstract}
A thesis submitted to the Faculty of Graduate and Postdoctoral
Affairs in partial fulfillment of the requirements for the degree of
\end{abstract}

Master of Applied Science

In

Electrical \& Computer Engineering

Carleton University

Ottawa, Ontario

(C) 2021

Rene Nyangezi 


\section{Abstract}

Shielding refers to the ability of a material or structure to attenuate electromagnetic waves from one region of space to another. This thesis looks at the design and construction of a door and frame assembly to improve its shielding effectiveness (SE) to meet the minimum specification of $50 \mathrm{~dB}$ from $1 \mathrm{GHz}$ to $28 \mathrm{GHz}$. This is accomplished by first looking at the choice of materials and construction of the assembly. To predict the performance of the design chosen, ANSYS HSSS (EM simulator) is used to look at the SE of the structure in an ideal environment. Shielding effectiveness measurements are performed on a miniaturized version of the design in an anechoic chamber. The performance of the final design is tested at Raymond EMC. This is accomplished by installing the full-size structure in an enclosure rated for $100 \mathrm{~dB}$ and performing the SE measurements in accordance with IEEE 299 standard. 


\section{Acknowledgments}

I would like to thank Professor Jim Wight for his guidance and continued support during my time at Carleton University. He has been a great mentor and without him, I would not be here. He has inspired me to think broadly and think of the bigger picture, beyond just academics. I would also like to thank Professor Rony Amaya for his help and continued belief in my skills and potential.

I also want to thank Ben Neil from Ambico for the assistance he has provided on 3D modeling and assembly work. This work would not have been possible without his contributions.

Thank you to all the people at Raymond EMC for helping in testing and assembly. I appreciate how accommodating they have been during the whole process.

I would like to thank the Department of Electronics at Carleton University for all the support they have provided me all these years.

I would also like to thank my friends and family for their continued support. Specifically, my mother Francoise Karangwayire and my friend Sandrine M. 


\section{Contents}

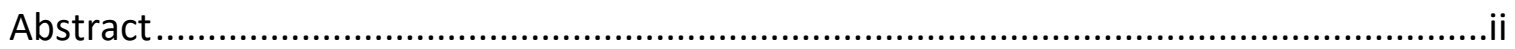

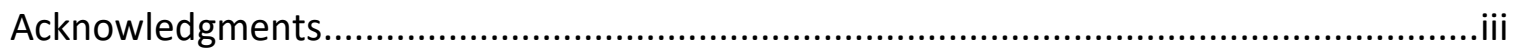

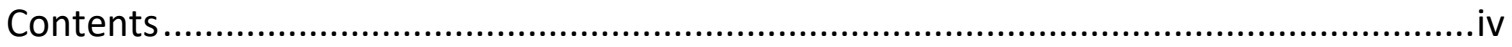

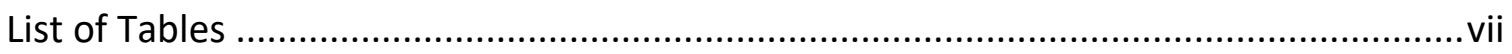

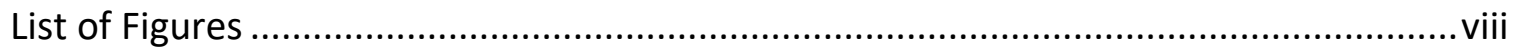

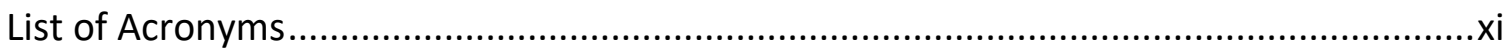

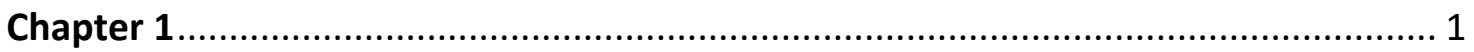

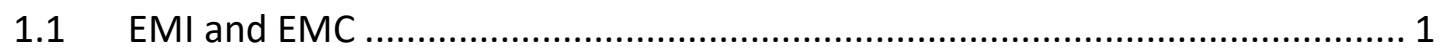

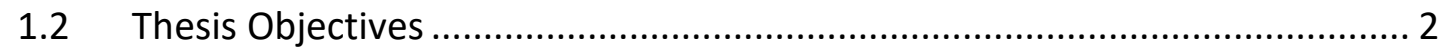

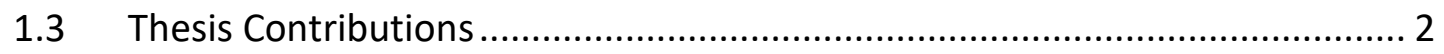

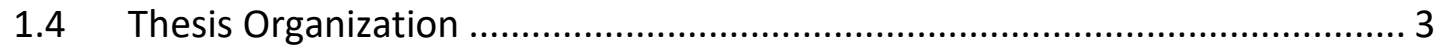

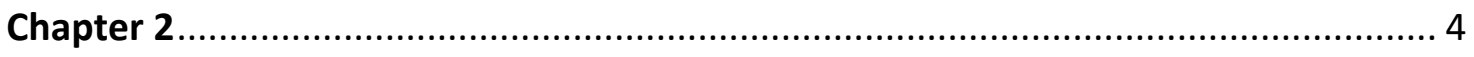

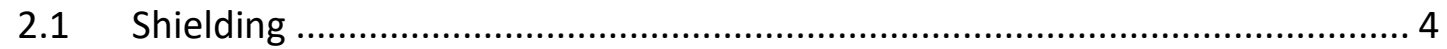

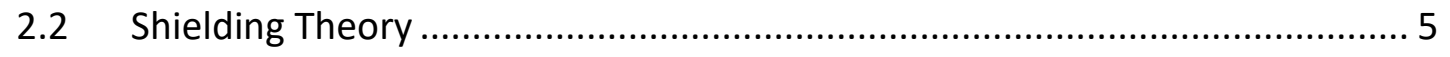

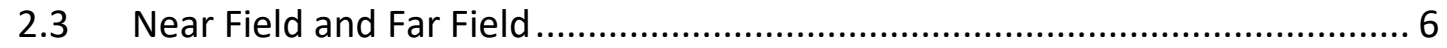

$2.4 \quad$ Shielding Effectiveness ...................................................................... 7

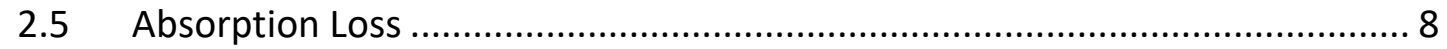

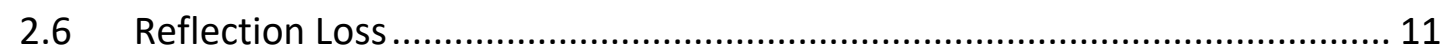




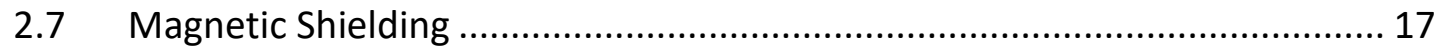

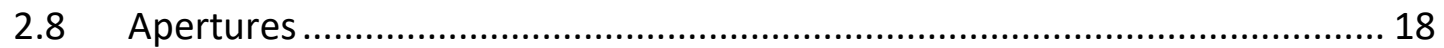

2.9 Seams

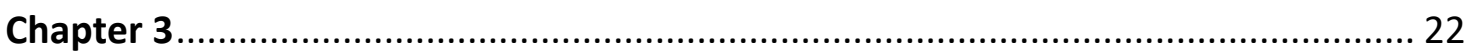

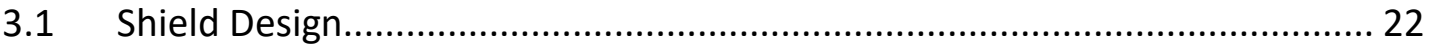

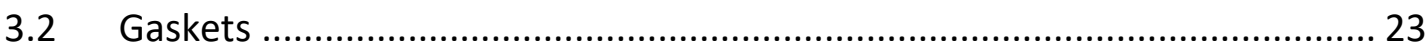

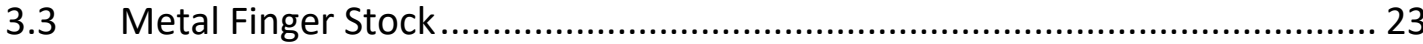

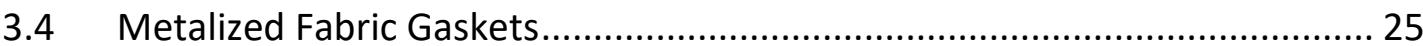

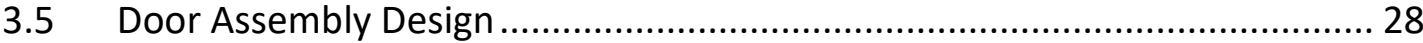

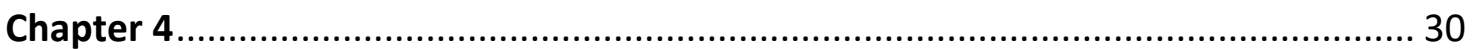

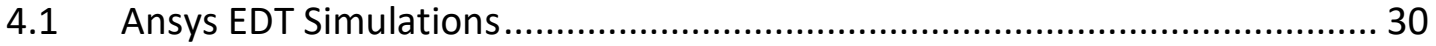

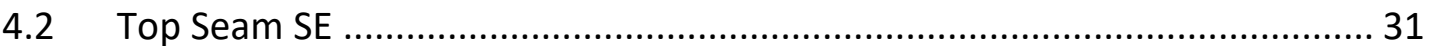

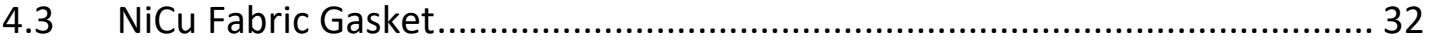

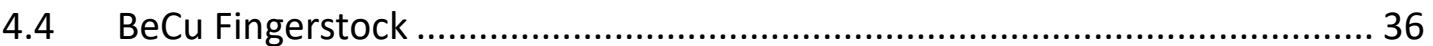

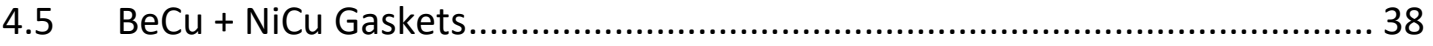

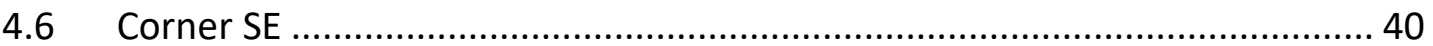

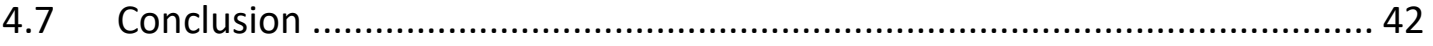

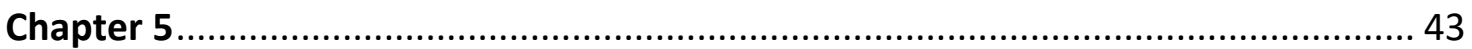

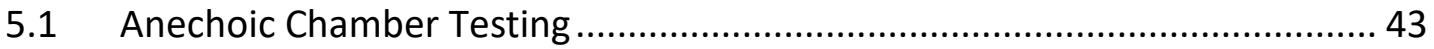




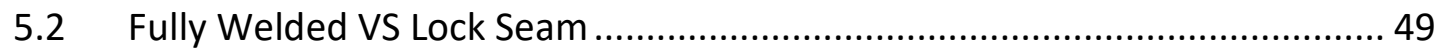

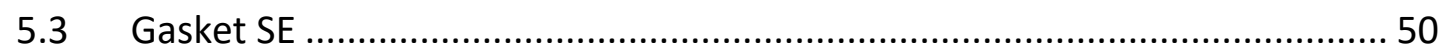

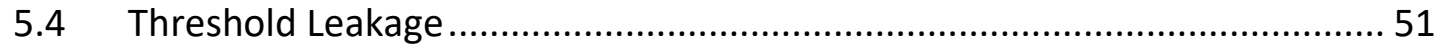

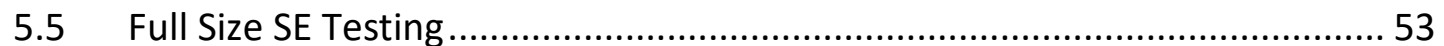

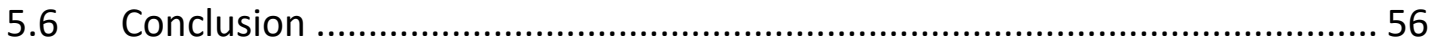

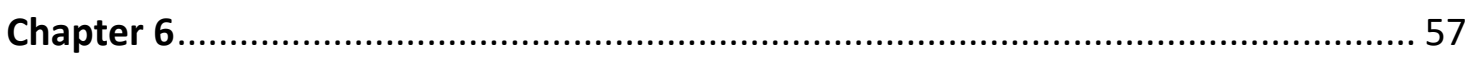

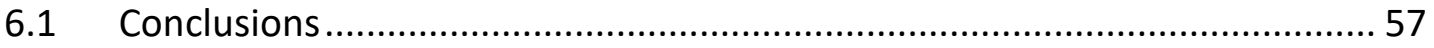

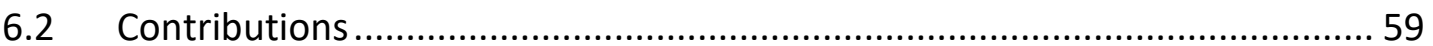

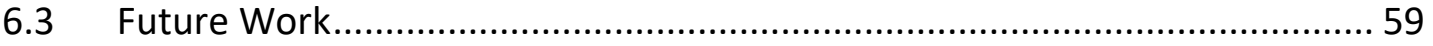

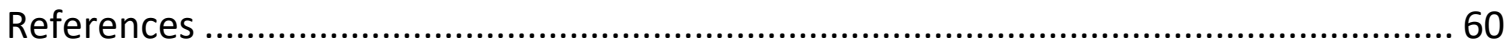




\section{List of Tables}

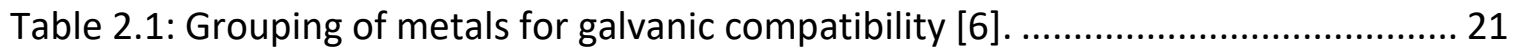

Table 5.1: List of antennas used in SE measurements............................................. 53 


\section{List of Figures}

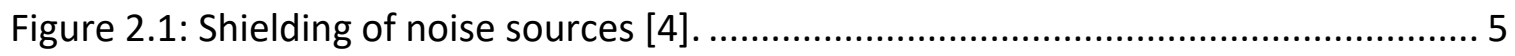

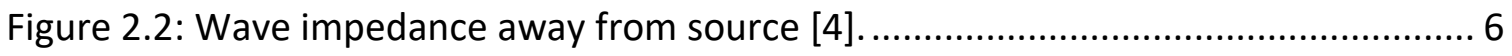

Figure 2.3: Electric field absorption in a medium [4] . ............................................... 9

Figure 2.4: Wave absorption as a function of the ratio of thickness to skin depth [4]... 10

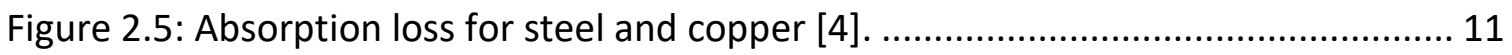

Figure 2.6: Partial reflection and transmission of incident E-field [4] ......................... 12

Figure 2.7: Partial reflection and transmission at 2 boundaries of shield [4]. ................ 13

Figure 2.8: Reflection loss for aluminum, copper, and steel [4]................................. 15

Figure 2.9: Multiple reflections in thin shields ..................................................... 15

Figure 2.10: Reflection loss correction factor for thin shields [4] ............................. 16

Figure 2.11: Magnetic shielding with ferromagnetic material [4] ................................ 17

Figure 2.12: Effects of discontinuities on induced currents, A) induced currents on shield, B) deviation of currents on shield with aperture, C) deviation of currents with thin

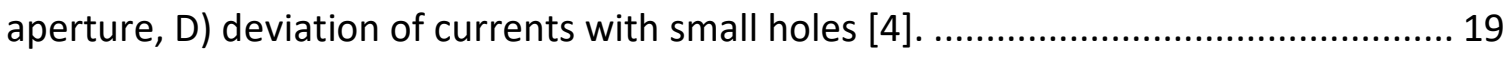

Figure 2.13: Measurement of transfer impedance of a joint [4]................................ 21

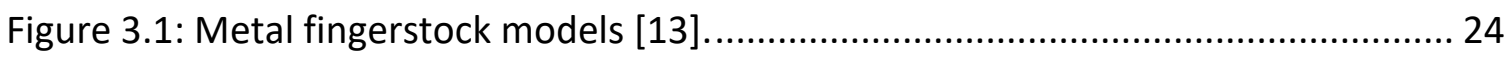

Figure 3.2: Alignment of surfaces and compression levels [11] ................................ 25

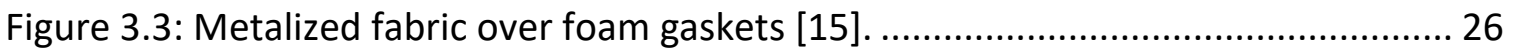

Figure 3.4: Gasket SE for $25 \%$ and 50\% compression [14]...................................... 27

Figure 3.5: 3D rendering of door and frame assembly with honeycomb core [18]....... 29

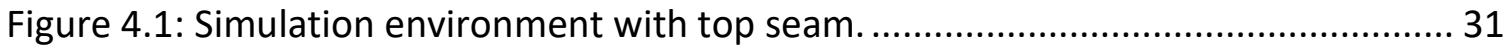


Figure 4.2: Top seam SE.

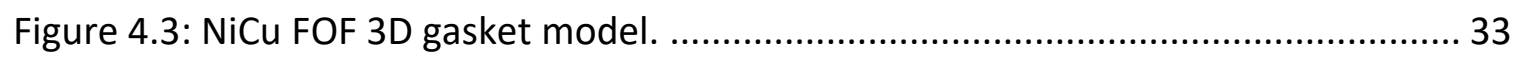

Figure 4.4: NiCu FOF gasket placement at the top seam. ........................................ 33

Figure 4.5: SE of NiCu FOF gasket for front, middle and back location......................... 34

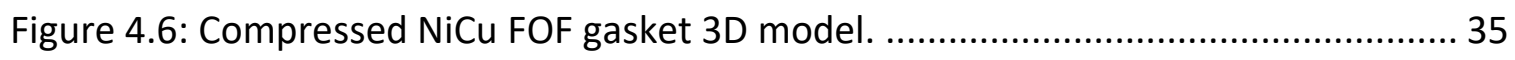

Figure 4.7: Compression comparison of BeCu fingerstock...................................... 35

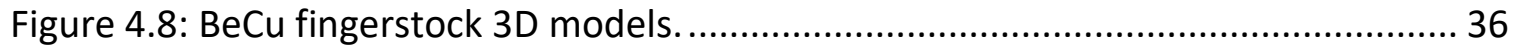

Figure 4.9: Compression comparison of BeCu fingerstock....................................... 37

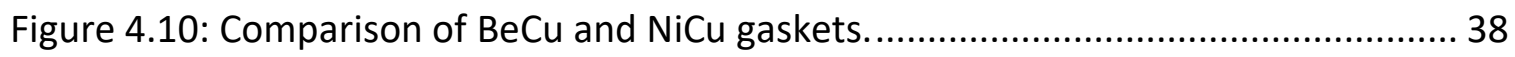

Figure 4.11: Comparison of $\mathrm{NiCu}-\mathrm{BeCu}$ and $\mathrm{BeCu}-\mathrm{NiCu}$ gasket placement. .................... 39

Figure 4.12: Comparison of all 4 cases of gasket configuration. ................................. 40

Figure 4.13: Corner illumination of the door frame. .............................................. 40

Figure 4.14: Comparison of SE of the corner and top seam....................................... 41

Figure 4.15: SE comparison of corner and top seam with same gasket configuration.... 41

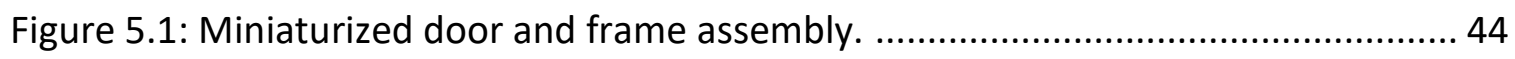

Figure 5.2: Section A-A of Miniaturized frame assembly. ....................................... 45

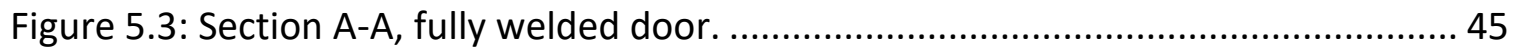

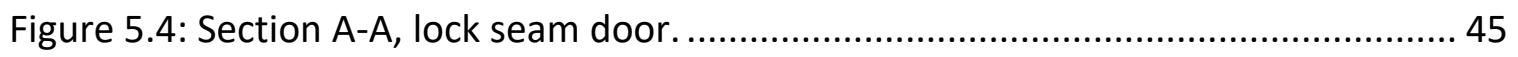

Figure 5.5: Section B-B of Miniaturized frame assembly ......................................... 46

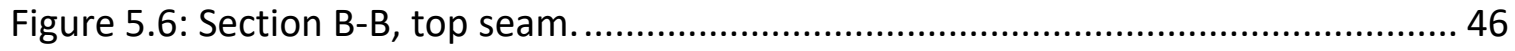

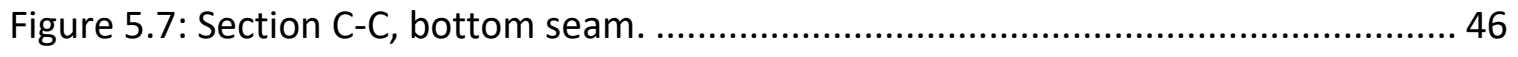

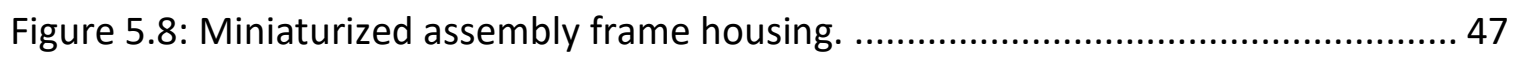


Figure 5.9: Miniaturized door and frame.

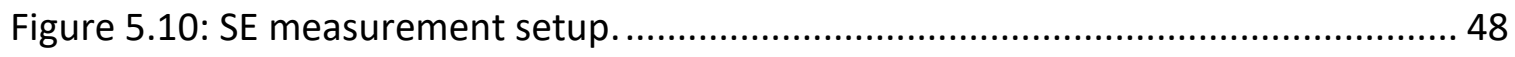

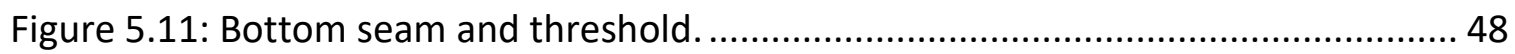

Figure 5.12: SE of fully welded and lock seam door in the anechoic chamber............... 49

Figure 5.13: Difference in SE between fully welded and lock seam door. ..................... 50

Figure 5.14: Comparison of SE of FOF only and both gaskets. .................................. 51

Figure 5.15: Difference in SE between FOF and both gaskets.................................... 51

Figure 5.16: Comparison of SE between the door with and without threshold. ............ 52

Figure 5.17: Comparison of SE between copper sealed and unsealed threshold........... 53

Figure 5.18: SE measurement locations around the door assembly............................ 55

Figure 5.19: Improvement in SE of this work and the prototype................................ 55 


\section{List of Acronyms}

$3 D$

CAD

EDT

EMC

EMI

FEM

MRI

PVC

RF

SCIF

SE three dimensional

computer aided design

electronic desktop

electromagnetic compatibility

electromagnetic interference

finite element method

magnetic resonance imaging

polyvinyl chloride

radio frequency

sensitive compartmented information facility

shielding effectiveness 


\section{Chapter 1}

\section{Introduction}

\subsection{EMI and EMC}

Electromagnetic interference (EMI) refers to the effects that outside sources have on the functionality of electrical systems [1]. These sources can be manmade or naturally occurring phenomena. In the case of manmade, it could be the transmission of radio waves or the transients caused by power machines like motors. Lightning and galactic noise would be examples of naturally occurring sources of EMI [1], [4]. The effects of EMI on electrical systems can range from minor, such that the system still performs as intended or catastrophic failure.

To prevent the unwanted effects of EMI, appropriate shielding must be implemented. This can be a metallic enclosure around the system to reflect and absorb most of the radiated energy or using a filter in the conductive path.

Electromagnetic compatibility (EMC) refers to the ability of a system to function properly in its environment while satisfying two criteria [1], [4]:

1. It does not cause interference with other systems.

2. It has adequate immunity to EM radiation from other sources.

The first criteria looks at the emissions whether conducted or radiated that would interfere with other systems. In general, some regulations specify the level of emissions 
acceptable based on the class of instrument. To meet this criterion, systems must undergo emissions testing.

The second criteria looks at how well a system can function in its environment in the presence of emissions from other sources. The immunity level of a system is the EM environment in which it can operate satisfactorily with a defined margin of safety.

\subsection{Thesis Objectives}

The thesis has two objectives:

1. Improve the Shielding effectiveness of the door and frame assembly to meet the minimum specification of $50 \mathrm{~dB}$. This target was set by Ambico to compete in the marketplace.

2. Find methods of improving shielding effectiveness of the frame assembly while saving cost on the design and testing process.

\subsection{Thesis Contributions}

1. The use of $\mathrm{BeCu}$ fingerstock and NiCu fabric over foam gaskets combined to shield the seams. This is in comparison to the industry standard use of one $\mathrm{BeCu}$ gasket. The combination of gaskets resulted in a minimum improvement in SE of $5 \mathrm{~dB}$.

2. The design of a miniaturized door and frame assembly as a means of saving cost on the design process and testing. This allowed for the comparison in SE performance between fully welded and lock seam doors including the performance of the gaskets. 


\subsection{Thesis Organization}

Chapter 1 introduces concepts related to electromagnetic interference and gives descriptions of EMI and EMC. It also provides the thesis objectives, contributions as well as the thesis outline.

Chapter 2 covers the background and theory of shielding in a sense that progresses to the understating of the key components of shielding effectiveness. The components are mainly the effects of reflectivity and absorption of materials.

Chapter 3 looks at the design of a door and frame assembly for shielding. The appropriate materials are investigated. This includes the appropriate choice of metals for the assembly construction and core material for the door. This chapter also covers the gaskets available on the market for EMI shielding.

Chapter 4 introduces electromagnetic simulations that are used to investigate the SE of the suggested design. This includes simulations on the top seam and the improvement in SE when gaskets are used. It also covers the performance of the corner of the door. Lastly, the threshold at the bottom of the door is investigated for leakage.

Chapter 5 covers emissions testing of a miniature model of the door and frame in an anechoic chamber. The performance in SE of a lock seam and fully welded door design is investigated. The effect of using a single or two gaskets is tested to compare to the EM simulations. The leakage introduced by the threshold is also tested. 


\section{Chapter 2}

\section{Background}

\subsection{Shielding}

Radiofrequency (RF) shielding doors have been widely used in the commercial, medical, and military sectors to protect electronic and electronic systems from malfunctioning under EMI or to prevent the theft of sensitive data.

A magnetic resonance imaging machine (MRI) is an example of a system that requires shielding to operate properly. Not only does the MRI room require a shielding door but it also requires a shielded ceiling, floor, and walls [2]. Without a shielding room, MRIs cannot produce clear images of patients due to outside noise interfering with the sensitive sensors.

EMC and EMI testing laboratories use shielding rooms for hardware testing. These rooms are usually built with metal walls to provide maximum shielding which leaves the entry points as the potential sources of interference. Shielding doors provide the necessary isolation from outside noise when testing hardware in these rooms. This is crucial when stringent specifications need to be met.

Shielding doors have become increasingly more popular in the construction of sensitive compartmented information facility (SCIF) rooms [3]. A SCIF is an accredited area where sensitive and classified information can be handled and stored. To prevent the theft of such sensitive data, the entrance to the SCIF uses a shielded door. 


\subsection{Shielding Theory}

A shield is a structure that is used to attenuate EM energy between two regions in space [4]. In general, these shields are made of metals and the reason for this is discussed in the following sections. There are two general cases of shielding, one where a source is contained in a shield to prevent electromagnetic interference (EMI) with other systems. The other is when a system is in a shield to protect it from outside noise sources. These two cases are shown in Fig. 2.1.
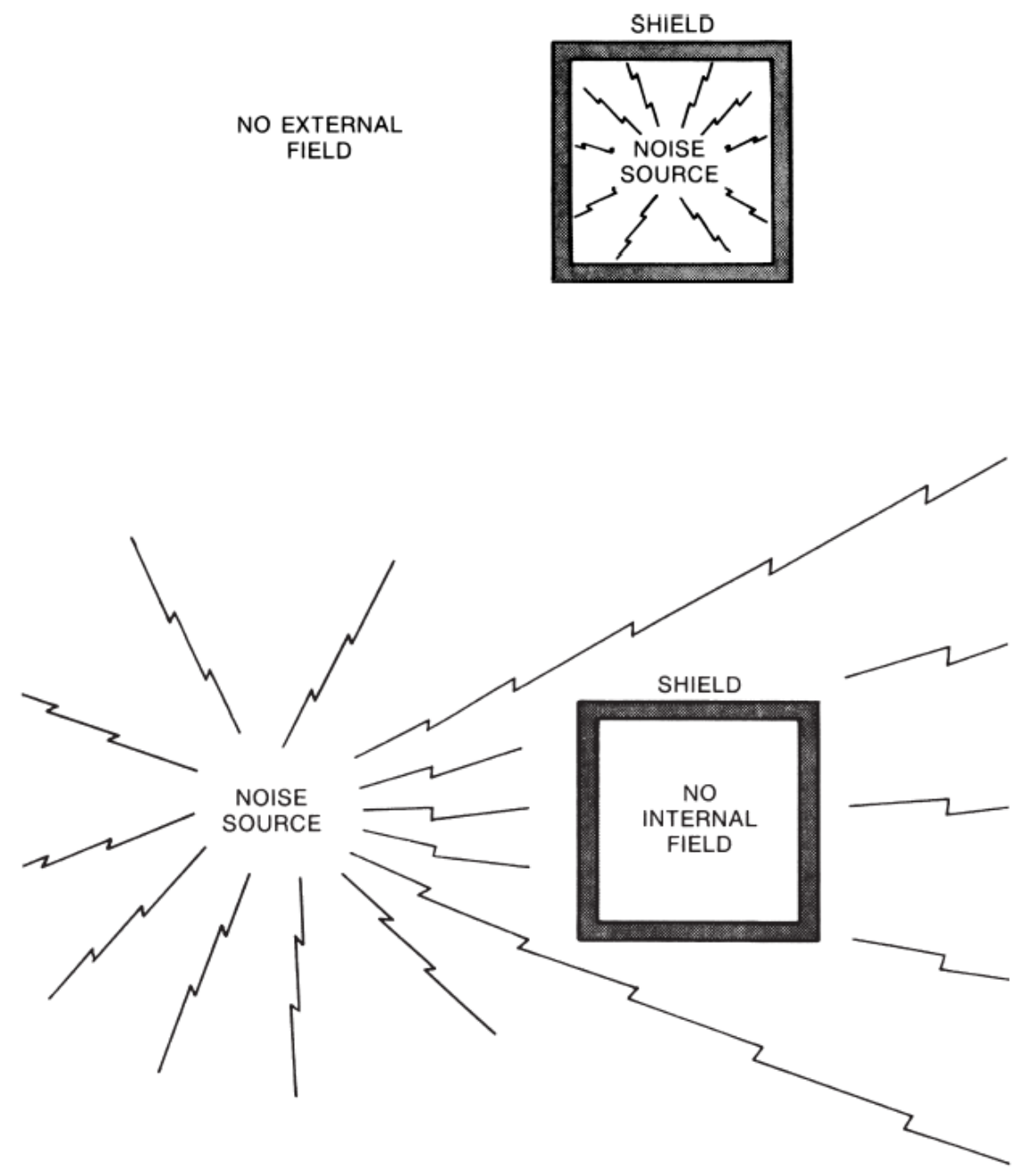

Figure 2.1: Shielding of noise sources [4]. 


\subsection{Near Field and Far Field}

The characteristics of the fields observed in space depend on the nature of the source and the distance from the source. In the area near the source (near field), less than $1 / 6$ of a wavelength, the fields will predominately be electric or magnetic [1], [4]. If the source uses high voltage and low current, then a predominantly electric field is produced in the near field. On the other hand, if the source is high current and low voltage then the field is magnetic in the same region. In both cases, the predominant field magnitude decreases at a rate of $(1 / r)^{3}$ rather than the usual $(1 / r)^{2}$ in the far-field. The ratio of the magnitude

of the electric (E) to magnetic $(\mathrm{H})$ field represents the wave impedance [4], [5]. Fig. 2.2 shows the two regions including the transition from near to far-field.

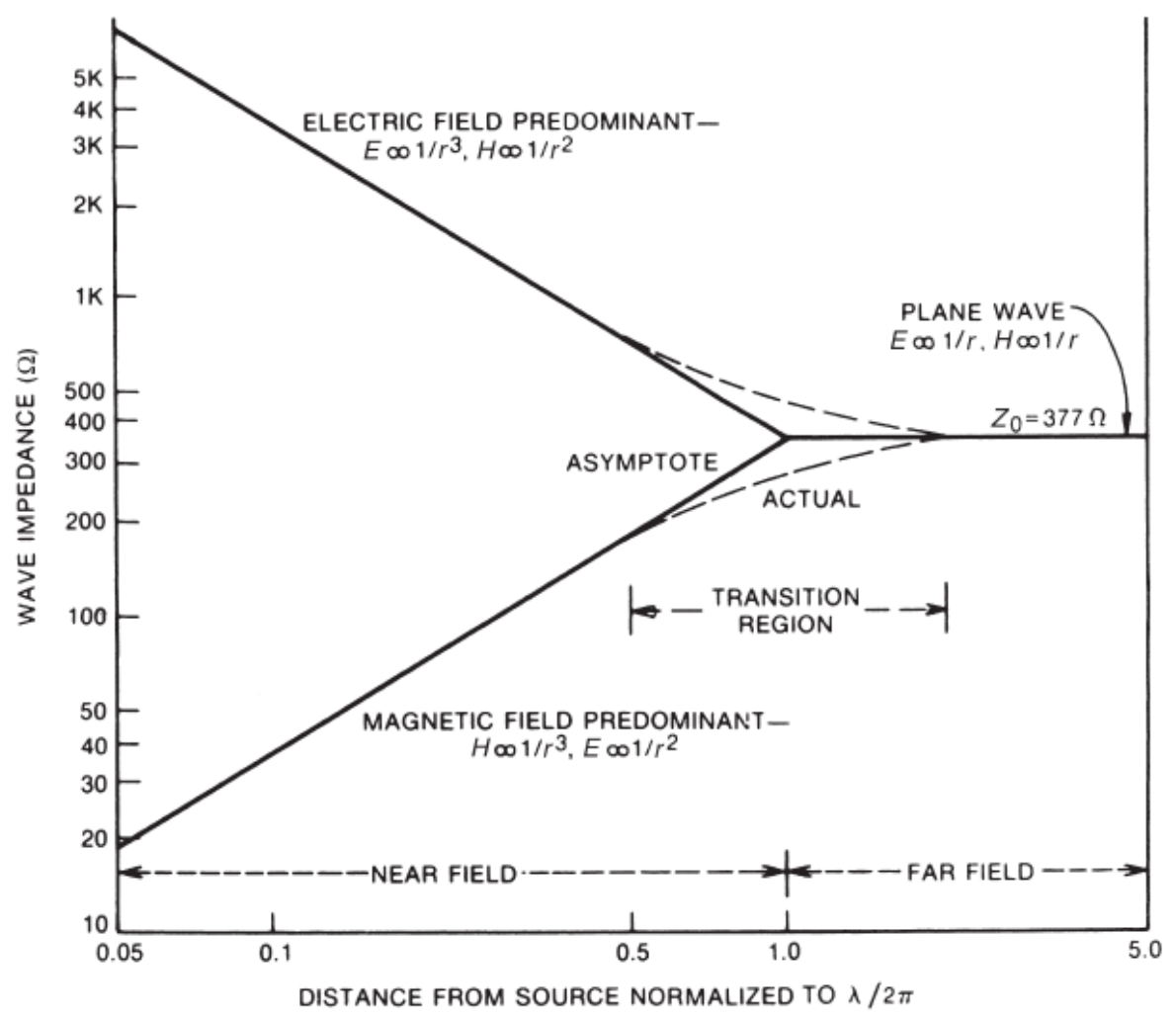

Figure 2.2: Wave impedance away from source [4]. 
In the far-field, the characteristics of the fields depend on the medium in which the fields are propagating. The three important characteristics of a medium that affect wave propagation are permeability $(\mu)$, dielectric constant $(\varepsilon)$, and conductivity $(\sigma)$ [4], [5]. As noted earlier the wave impedance of an electromagnetic wave is

$Z_{w}=\frac{\mathrm{E}}{\mathrm{H}}$

The characteristic impedance of a medium is defined as

$Z_{0}=\sqrt{\frac{j \omega \mu}{\sigma+j \omega \varepsilon}}$

In far-field, $Z_{w}=Z_{0} \approx 377 \Omega$ when considering plane wave propagation in free space.

In the case of a conductor were $(\sigma \gg j \omega \varepsilon)$, the characteristic impedance is referred to as the shield impedance $Z_{s}$ [4]. It is

$Z_{0}=\sqrt{\frac{j \omega \mu}{\sigma}}=Z_{s}$

\subsection{Shielding Effectiveness}

Shielding effectiveness (SE) is a measure of how well a material or structure can attenuate EM waves [4]. In general, SE is comprised of two components, wave reflection, and absorption. Wave reflection is due to the difference in wave impedance at the boundary of two different materials. Absorption measures the decay in the amplitude of an EM wave as it propagates into a material. 
The SE of a material is defined as the ratio of the incident electric or magnetic field to the transmitted field [1], [4]. This quantity is represented in decibels. The following equations show these relationships.

$S E=20 \log \left(\frac{E_{0}}{E_{1}}\right) d B$

$S E=20 \log \left(\frac{H_{0}}{H_{1}}\right) d B$

In the equations above, $E_{0}\left(H_{0}\right)$ represents the incident field strength and $E_{1}\left(H_{1}\right)$ the transmitted field.

\subsection{Absorption Loss}

When an EM wave passes through a media, its amplitude is decreased exponentially [4], [5]. The decrease in amplitude represents a transfer of energy from the wave to the media. This transfer is in the form of heat. The amplitude of the electric or magnetic field in the media relative to the incident field magnitudes are.

$E_{1}=E_{0} e^{\frac{-t}{\delta}}$

$H_{1}=H_{0} e^{\frac{-t}{\delta}}$

Where $E_{1}\left(H_{1}\right)$ are the field intensity at a distance $t$ within the material, and $\delta$ is the skin depth. The skin depth represents the distance at which the incident field magnitude is attenuated to roughly 37 percent. Fig. 2.3 shows the decay in field strength as a wave propagates in a medium. 

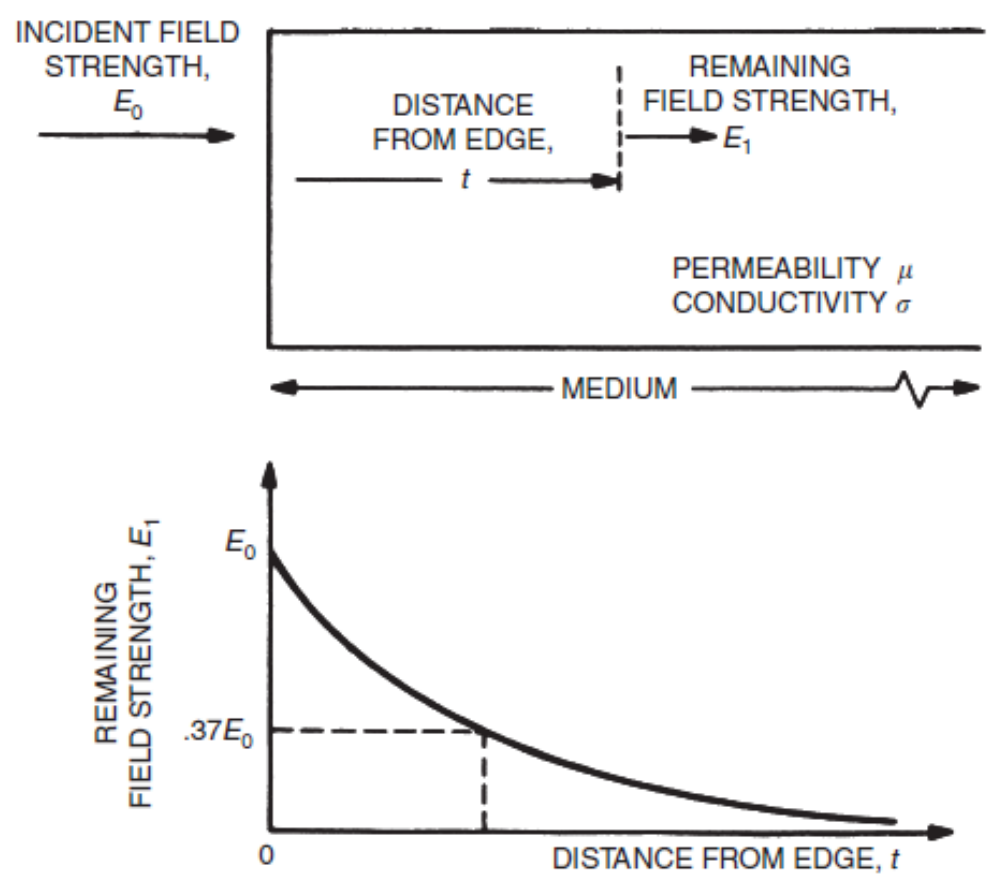

Figure 2.3: Electric field absorption in a medium [4].

The skin depth is material-specific and is frequency dependent as given by

$\delta=\sqrt{\frac{2}{\omega \mu \sigma}}$

The absorptions loss $(\mathrm{A})$ can be calculated as

$A=20 \log \left(\frac{E_{0}}{E_{1}}\right)=20 \log \left(e^{\frac{t}{\delta}}\right)=8.69\left(\frac{t}{\delta}\right)[d B]$

The equation above shows that a thickness of one skin depth results in an absorption loss of approximately $8.7 \mathrm{~dB}$. Fig. 2.4 shows the absorption loss as a function of the ratio $\left(\frac{t}{\delta}\right)$. This representation of absorption loss is only applicable in the far-field when plane waves are considered. 


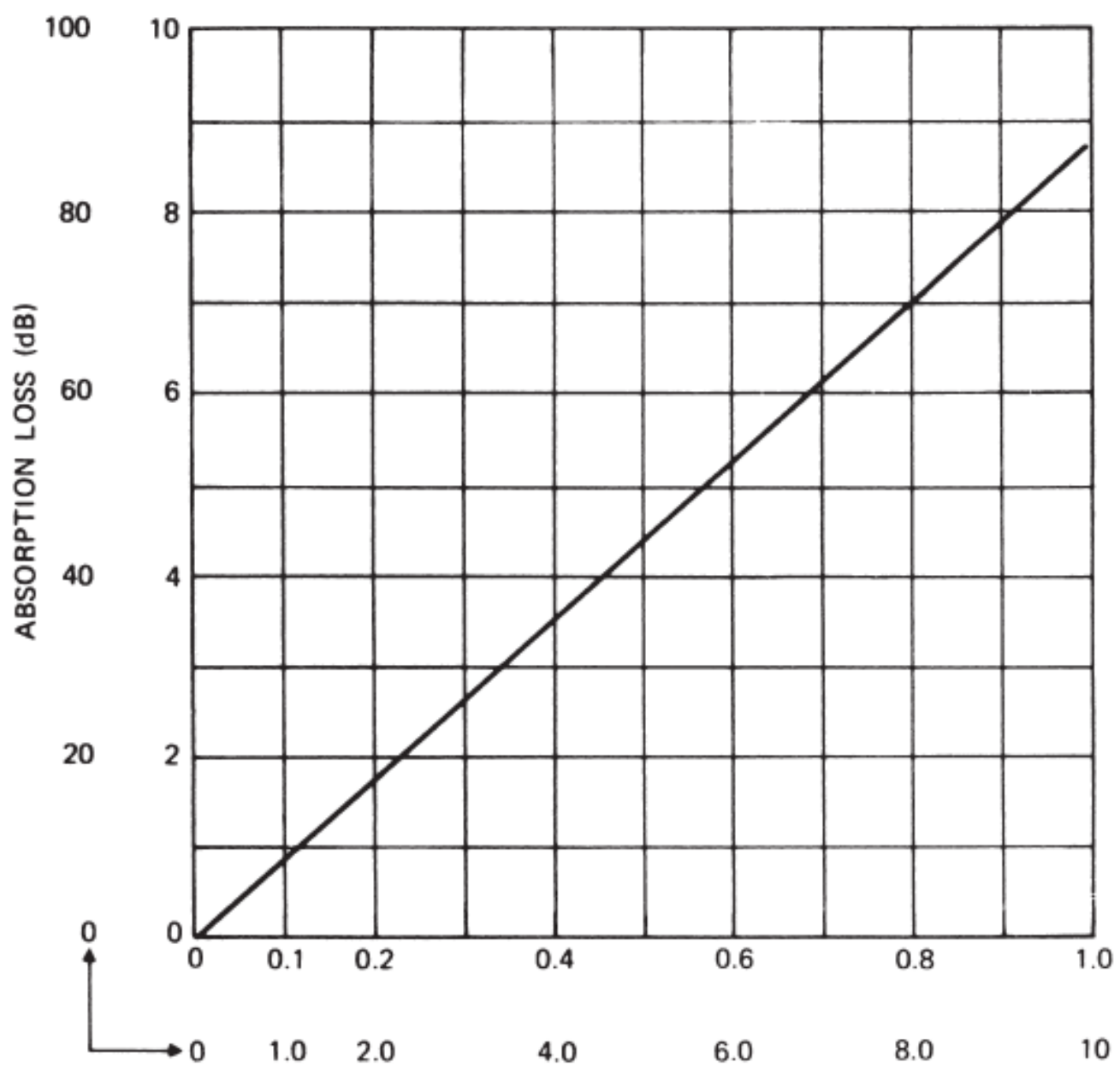

RATIO OF THICKNESS, $t$ TO SKIN DEPTH, $\delta$

Figure 2.4: Wave absorption as a function of the ratio of thickness to skin depth [4].

As shown in eq. 1.7, the skin depth is frequency-dependent and as a result, so is the absorption loss. This can be seen in Fig. 2.5, where the absorption loss is plotted for two thicknesses of steel and copper. 


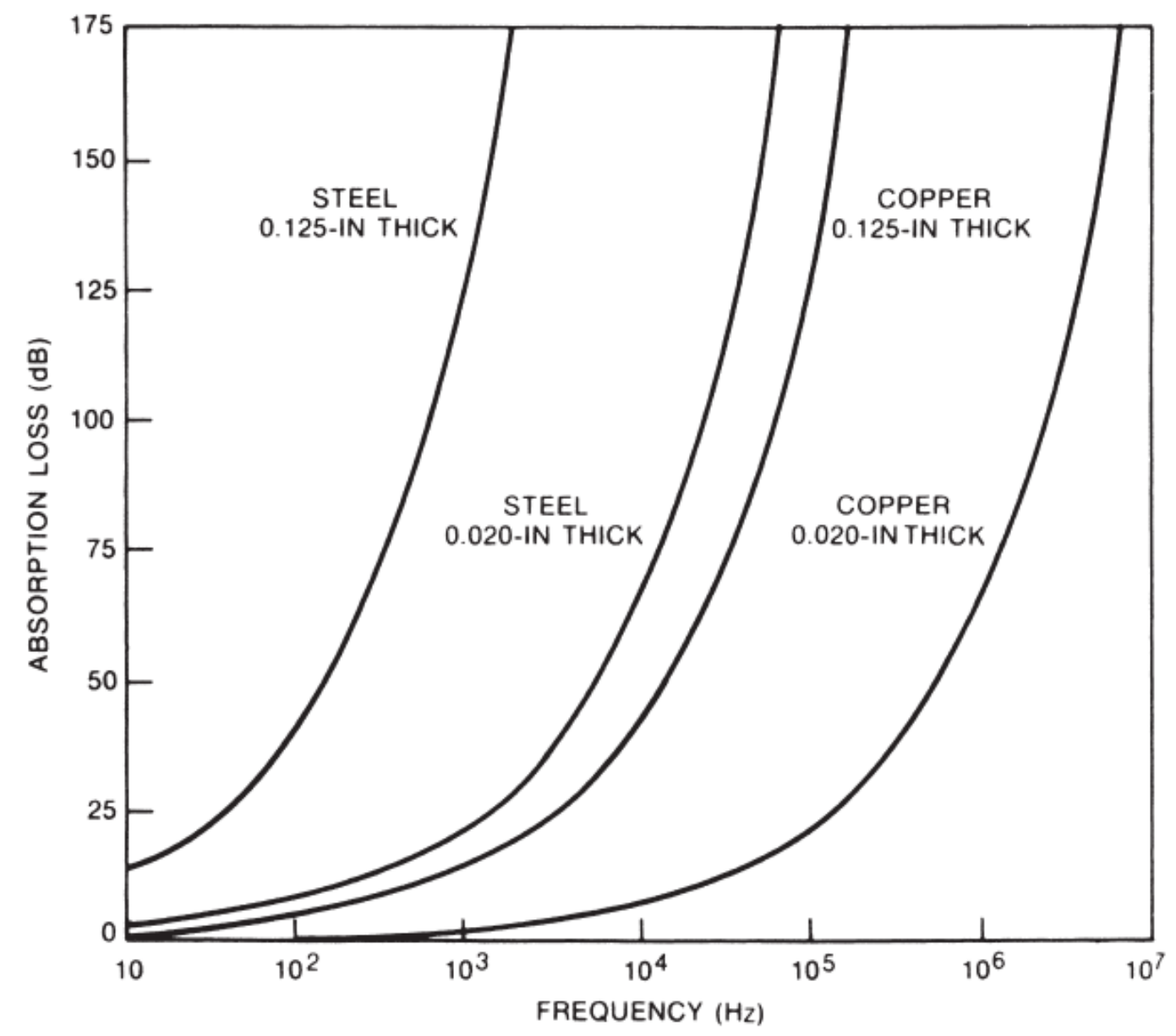

Figure 2.5: Absorption loss for steel and copper [4].

\subsection{Reflection Loss}

Reflection loss is the result of differences in characteristic impedances at the boundary between two media [4], [5]. This phenomenon is illustrated in Fig. 2.6 which includes the amplitudes of the incident, reflected, and transmitted waves.

$E_{0}$ is the incident wave, $E_{r}$ is the reflected wave, and $E_{1}$ is the transmitted wave.

The intensity of the transmitted wave in medium 2 is

$$
E_{1}=\frac{2 Z_{2}}{Z_{1}+Z_{2}} E_{0}
$$


With the relationships of impedances, $Z_{1}=\frac{E_{0}}{H_{0}}$ and $Z_{2}=\frac{E_{1}}{H_{1}}$ substituted in the equation Eq. 1.9, the transmitted magnetic field is

$H_{1}=\frac{2 Z_{1}}{Z_{1}+Z_{2}} H_{0}$

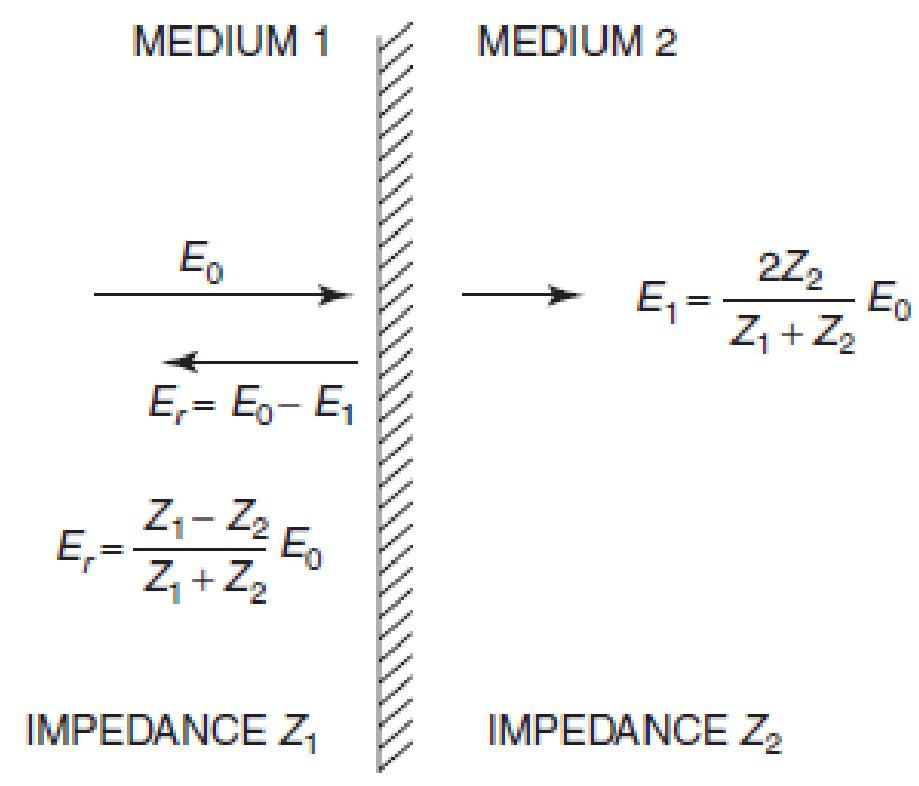

Figure 2.6: Partial reflection and transmission of incident E-field [4].

If a wave passes through a shield, it will encounter two boundaries as shown in Fig. 2.7. At the second boundary, the wave will be in a medium with impedance $Z_{2}$ and encounter a medium with impedance $Z_{1}$. The intensities of the transmitted electric and magnetic fields are given by

$E_{1}=\frac{2 Z_{1}}{Z_{1}+Z_{2}} E_{0}$

and

$H_{1}=\frac{2 Z_{2}}{Z_{1}+Z_{2}} H_{0}$ 
The total transmitted waves $E_{t}$ and $H_{t}$ through the shield in Fig. 2.7 can be obtained by substituting Eq. 1.9 and 1.10 into Eq. 1.11 and 1.12 and result in

$E_{t}=\frac{4 Z_{1} Z_{2}}{\left(Z_{1}+Z_{2}\right)^{2}} E_{0}$

and

$H_{t}=\frac{4 Z_{1} Z_{2}}{\left(Z_{1}+Z_{2}\right)^{2}} H_{0}$

This is valid for thick shields and neglects multiple reflections and neglects the absorption loss which can be account for by Eq. 1.8 .

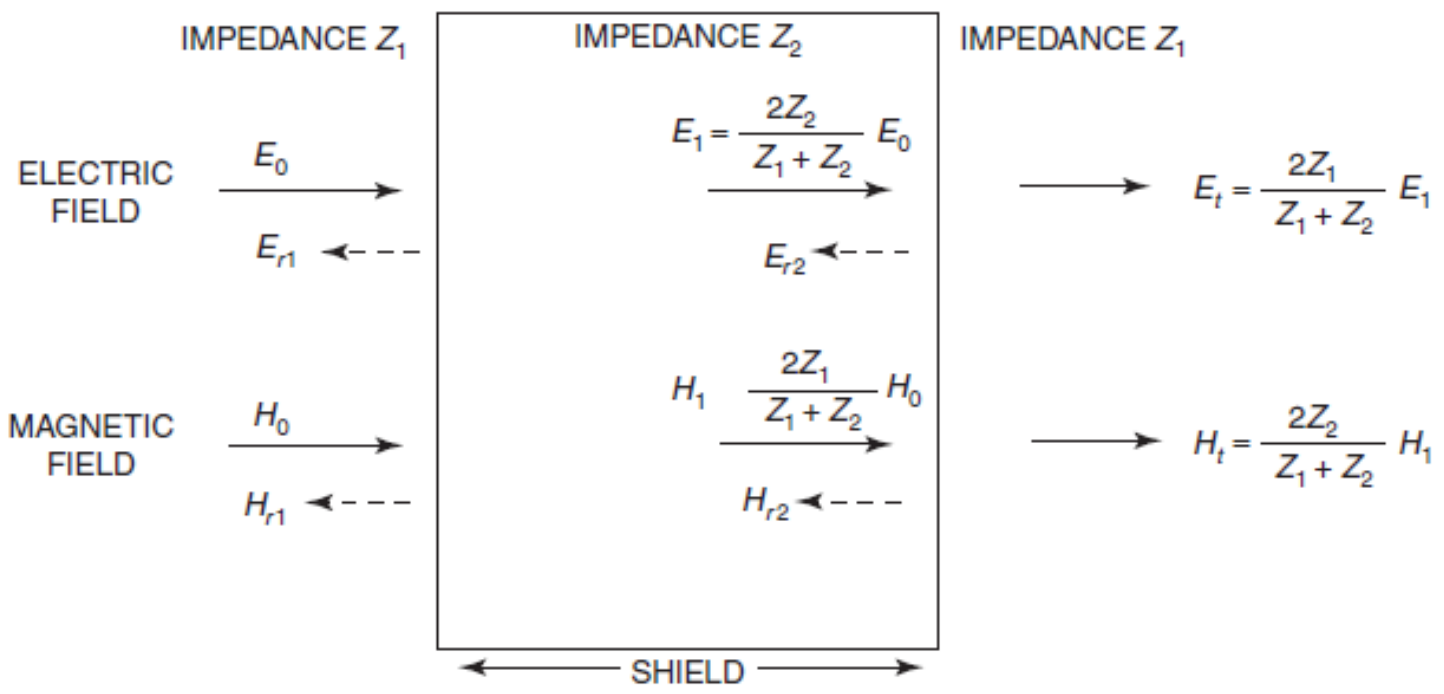

Figure 2.7: Partial reflection and transmission at 2 boundaries of shield [4].

If the shield is a good conductor surrounded by an insulator then $Z_{1} \gg Z_{2}$, this can be seen from Eq. 1.2 and 1.3. This results in Eq 1.13 and 1.14 reducing to 
$E_{t}=\frac{4 Z_{2}}{Z_{1}} E_{0}$

and

$H_{t}=\frac{4 Z_{2}}{Z_{1}} H_{0}$

By substituting the wave impedance $Z_{w}$ for $Z_{1}$ and the shielding impedance $Z_{s}$ for $Z_{2}$ the reflection loss can be calculated as

$R=20 \log \left(\frac{E_{0}}{E_{1}}\right)=20 \log \frac{\left|Z_{w}\right|}{\left|Z_{S}\right|} d B$

By substituting for plane waves in free space with $Z_{w}=Z_{0} \approx 377 \Omega$ and for $Z_{s}$ from Eq. 1.3 then Eq. 1.17 becomes

$R=20 \log \frac{94.25}{\left|Z_{s}\right|}=168+10 \log \left(\frac{\sigma_{r}}{\mu_{r} f}\right) d B$

where $\sigma_{r}$ is the relative conductivity compared to copper $\left(\sigma_{\text {copper }}=5.82 \times\right.$ $10^{7}$ siemens $/ m$ )

The reflection loss in Eq. 1.17 is plotted in Fig. 2.8 for three common shields as a function of frequency. The absorption loss discussed in the previous sections in Fig. 2.5 showed that steel had a much higher loss compared to copper but in the case of reflection, copper has approximately $30 \mathrm{~dB}$ more attenuation. 


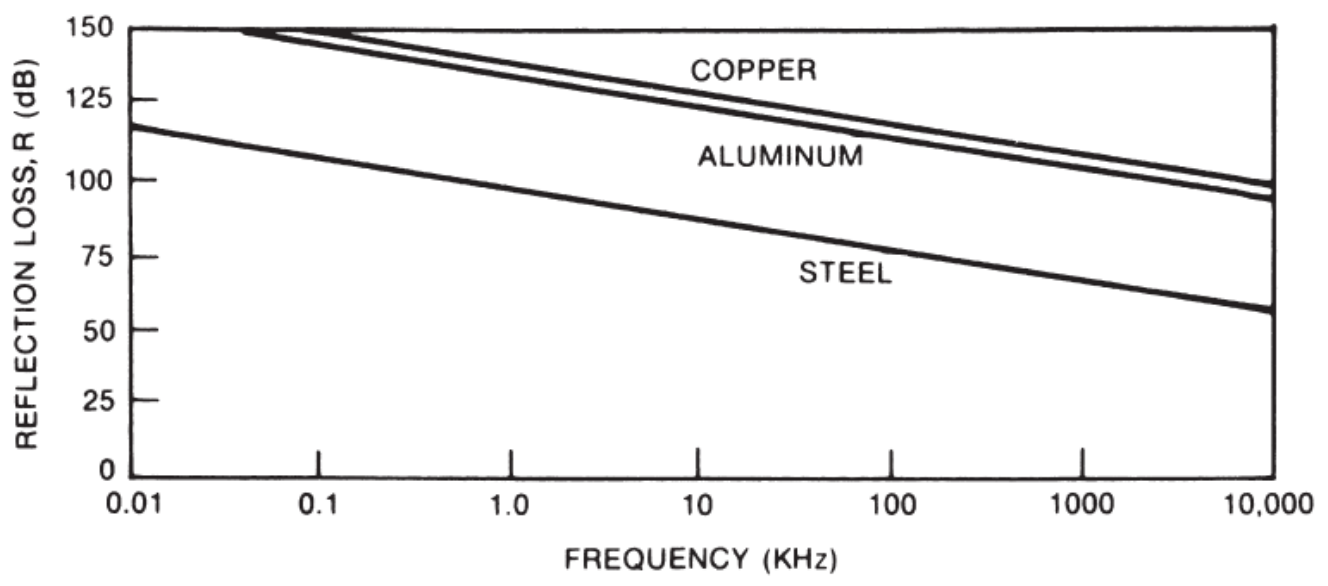

Figure 2.8: Reflection loss for aluminum, copper, and steel [4].

Multiple reflections will occur in thin shields as shown in Fig. 2.9. This is only relevant for the transmitted magnetic field intensity since the electric field is mostly reflected at the first boundary [1], [4]. This can be seen from Eq 1.9. The remaining electric field in the shield is negligible since the absorption loss is high. As mentioned previously since $Z_{1} \gg$ $Z_{2}$ for a shield, the transmitted magnetic field is doubled as shown in Eq 1.10. With the intensity doubled, multiple reflections must be considered for thin shields.

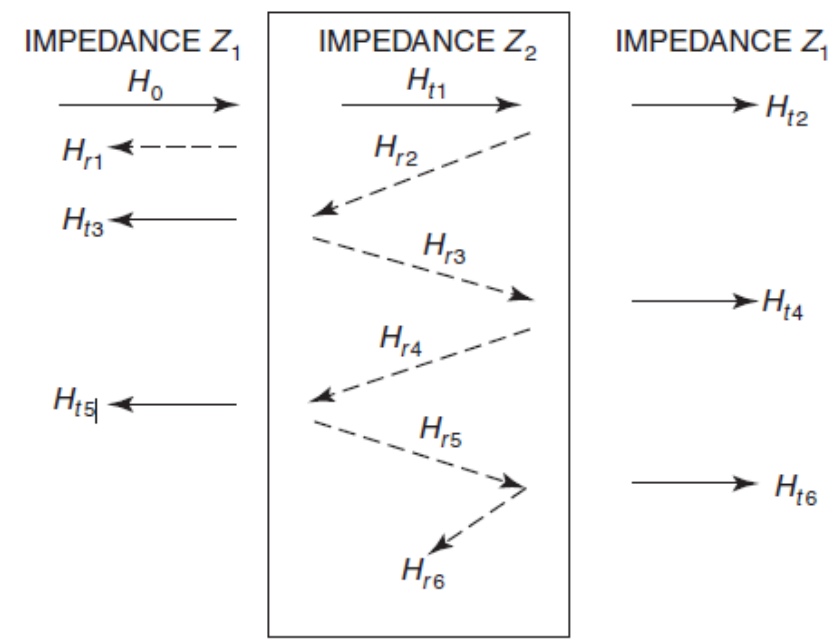

Figure 2.9: Multiple reflections in thin shields 
A correction factor to the reflection loss must be added for the case of thin shields due to multiple reflections given by

$B=20 \log \left(1-e^{\frac{-2 t}{\delta}}\right) d B$

This correction term is plotted below in Fig. 2.10.

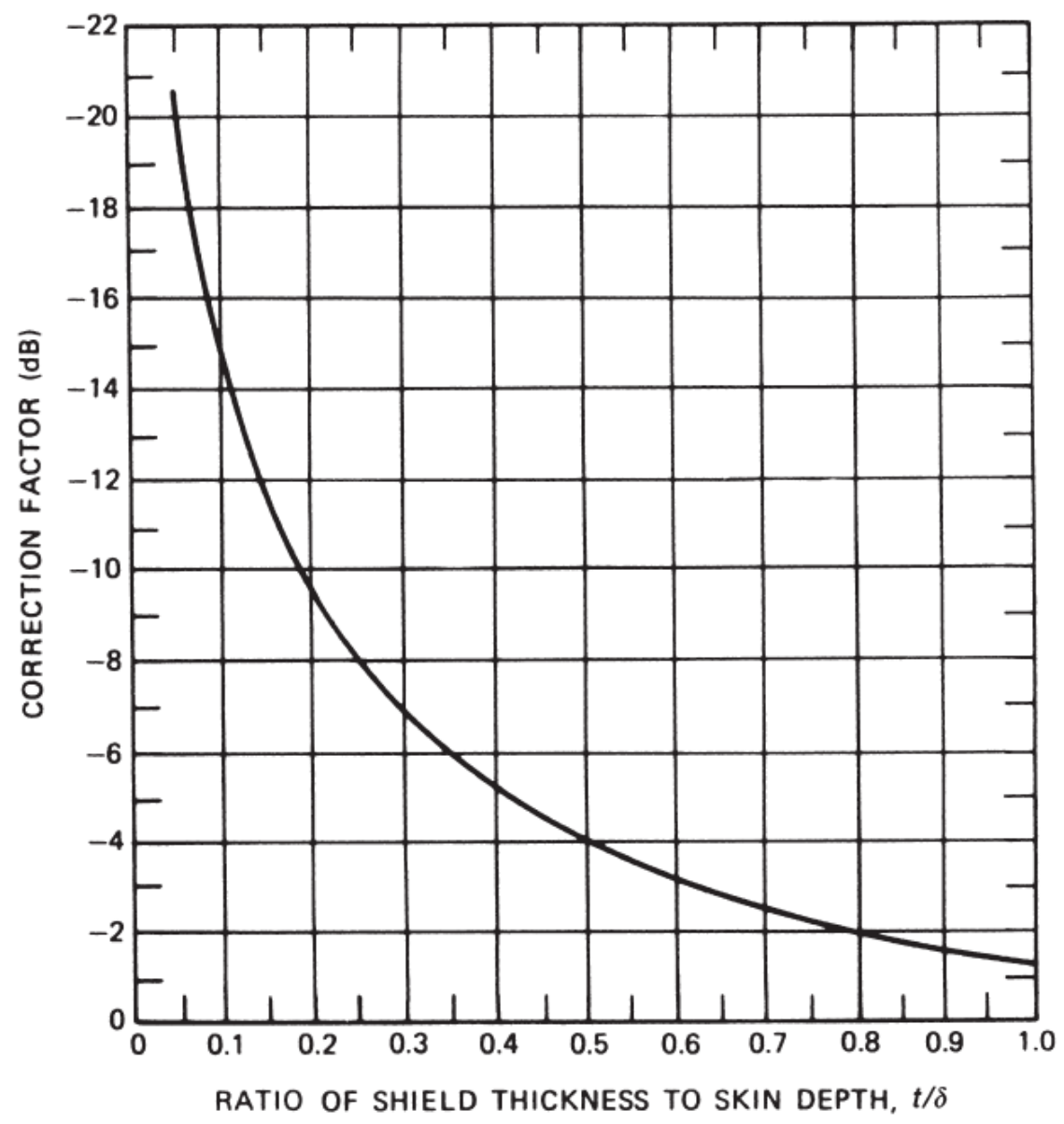

Figure 2.10: Reflection loss correction factor for thin shields [4].

The total SE of a material with no apertures can be calculated as the sum of the absorption loss, reflection loss, and the correction factor for thin shields as

$S E=A+R+B d B$ 


\subsection{Magnetic Shielding}

In the near field, the loss for low-frequency magnetic fields is low [4]. This includes the summation of the absorption and reflection loss. In general, the dominant loss for magnetic fields at all frequencies is the absorption loss [4]. To effectively shield against low-frequency magnetic fields, a ferromagnetic material must be used [1], [4]. This means a material with $\mu_{r} \gg 1$. The way that ferromagnetic materials work in shielding lowfrequency magnetic fields is shown below in Fig. 2.11. The material provides a low reluctance path which will concentrate the magnetic field lines [4], [5]. As a result, this will divert the magnetic field from the shielded region.

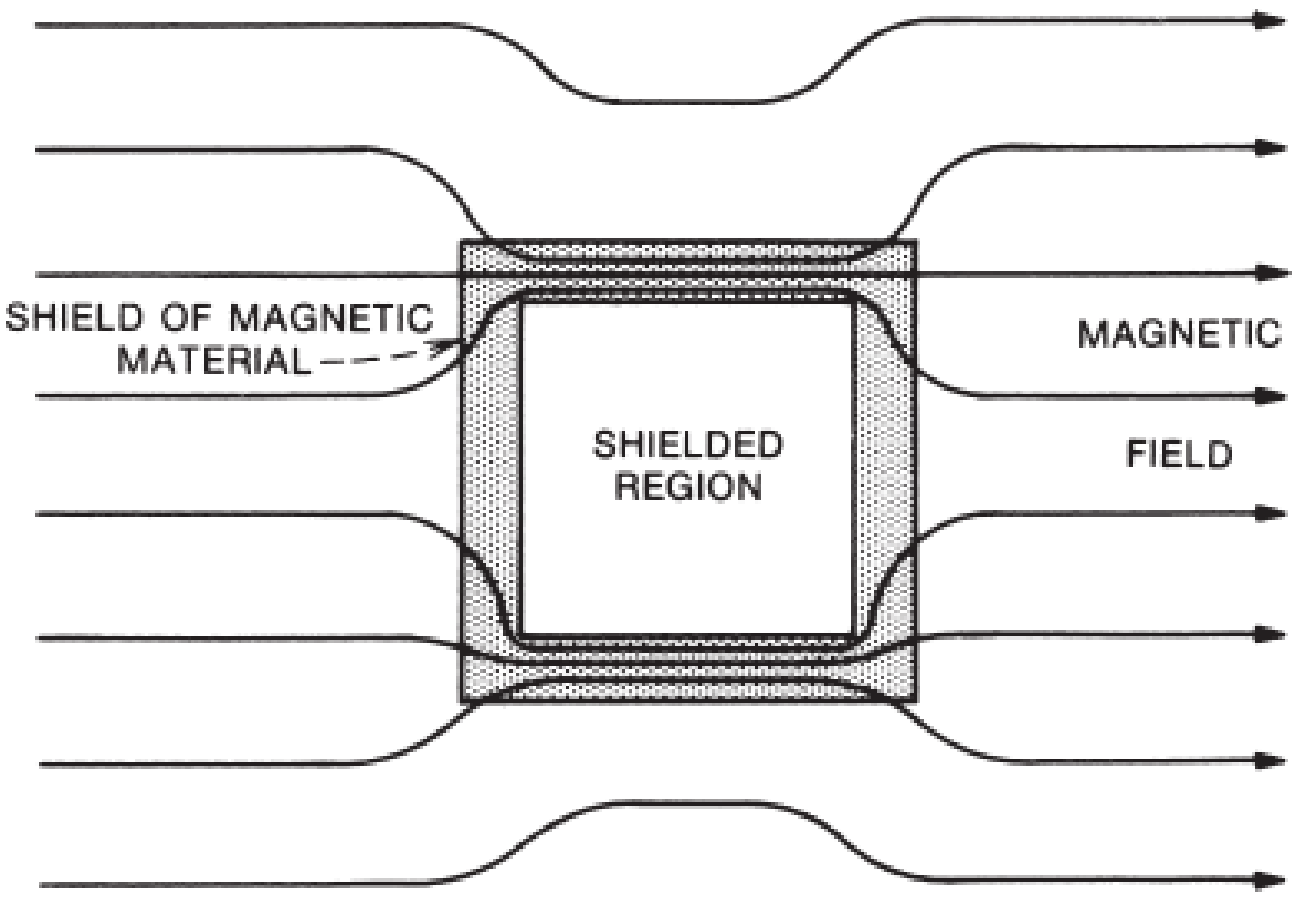

Figure 2.11: Magnetic shielding with ferromagnetic material [4]. 


\subsection{Apertures}

The shielding theory discussed thus far has only been for continuous shields without apertures. In general, shielding enclosures require doors and holes for cables or ventilation [1], [4]. These apertures in most cases will greatly reduce the shielding effectiveness of a structure. The amount of the leakage introduced by an aperture is mainly dependent on the maximum linear dimension and the frequency of the incident EM wave [4]. The simplest way to think of how an aperture affects the SE of a metal is to look at the surface currents. When an incident EM wave encounters a shield, it produces surface currents as shown in Fig. 2.12A, [4]. These surface currents will produce an electric field that is of opposite polarity to meet the tangential boundary condition [4], [5]. This boundary condition requires that the tangential electric field be zero for a perfect conductor. Thus, canceling the incident electric field. It is important to note that none of the materials discussed previously are perfect conductors and this led to the idea of skin depth introduced previously. A perfect conductor would have $\delta=0$ since $\sigma=\infty$ as noted from Eq. 1.7. When an aperture is introduced in the material as in Fig. 2.12B, the current is forced around the aperture. This deviation in current results in lower shielding effectiveness since the current required to cancel the incident electric field is no longer present within the area of the aperture [4]. A narrower slot is shown in Fig. 2.12C compared to Fig. 2.12B but the resulting current deviation is roughly the same. This results in the same amount of leakage even though the area has been greatly reduced and this points to the importance of the maximum linear dimension of the aperture. By replacing the slot with multiple holes as in Fig. 2.12D, the deviation in the currents has 
been greatly reduced and thus so has the leakage even though the overall area is the same.

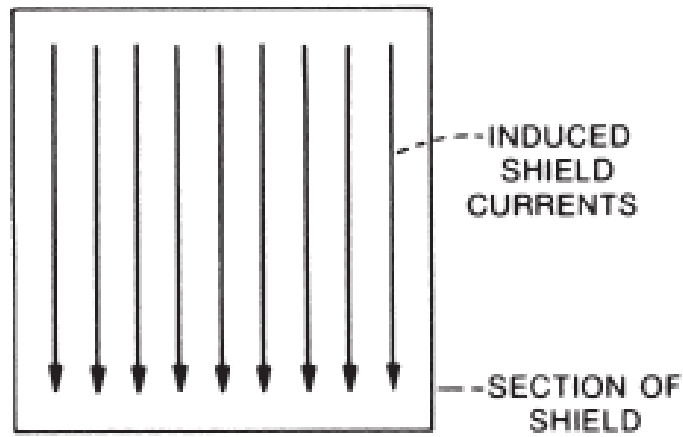

A

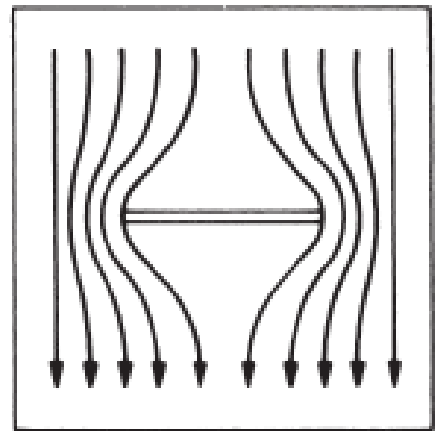

$\mathrm{C}$
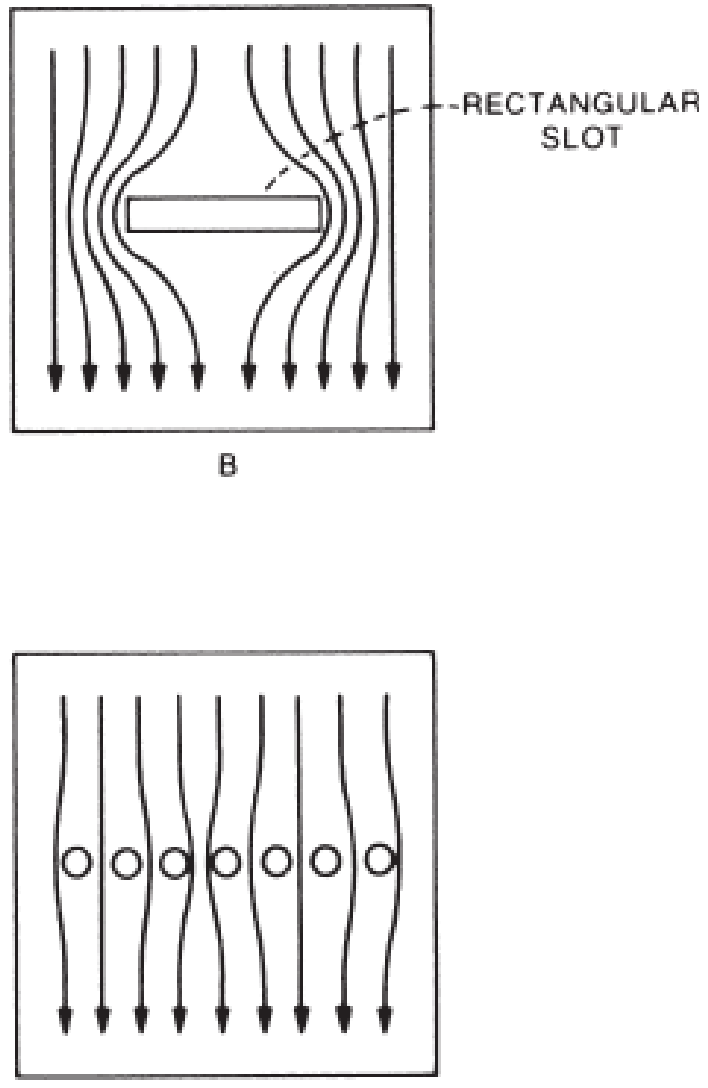

D

Figure 2.12: Effects of discontinuities on induced currents, A) induced currents on shield,

B) deviation of currents on shield with aperture, C) deviation of currents with thin aperture, D) deviation of currents with small holes [4]. 


\subsection{Seams}

Some enclosures require doors for accessibility. This results in a seam, which is a long narrow slot between the door and the rest of the enclosure. Even though a seam can have a thickness of a few millimeters, it behaves like a radiating antenna [4]. In antenna theory, a seam with a length of the order of half a wavelength can be considered as a slot antenna. In general slot antennas are efficient radiators [4]. To reduce the radiating potential of a seam, good electrical continuity is required between its mating parts. This can be achieved by providing periodic contact across the length of the seam by using fasteners, contact fingers, and conductive gaskets [1], [4]. The shielding effectiveness of a seam is difficult to measure because it requires radiated emissions testing. A simpler method of finding the electrical contact quality is to measure the transfer impedance [4]. The test setup for transfer impedance measurements is shown in Fig. 2.13. The joint under test represents a seam with a conductive gasket. The transfer impedance is the ratio of the developed voltage across the joint to the current through it. The shielding effectiveness of a seam can be approximated with its shielding quality, which is

$S=20 \log \frac{\left|Z_{W}\right|}{\left|Z_{T}\right|}$

Where $Z_{T}$ is the transfer impedance and $Z_{W}$ is the wave impedance.

A properly designed seam should have a transfer impedance of a few milliohms [4]. The measurements in Fig. 2.13 can be obtained with the use of a signal generator and a spectrum analyzer since the transfer impedance is frequency-dependent. 


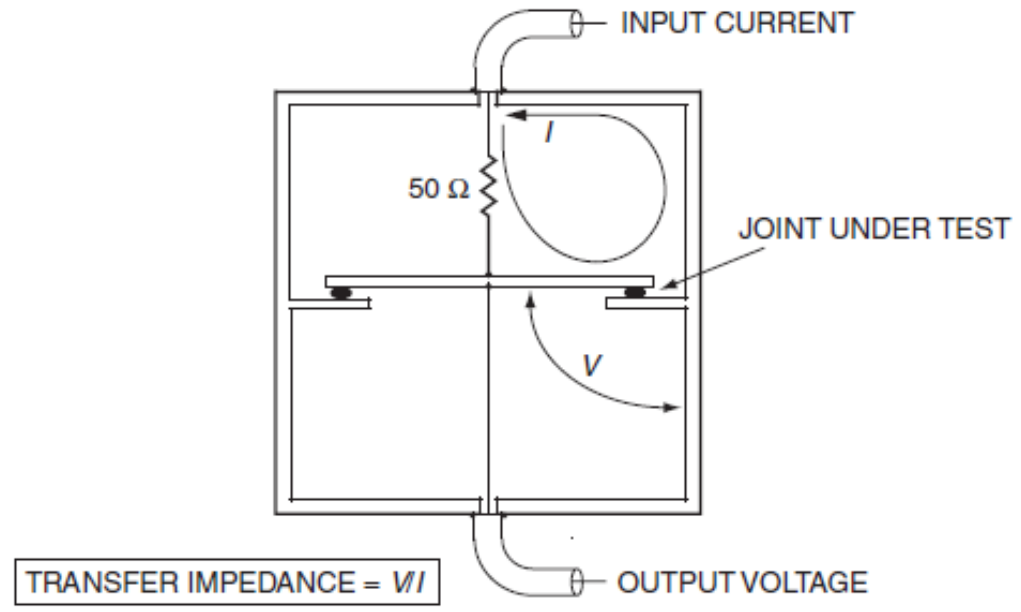

Figure 2.13: Measurement of transfer impedance of a joint [4].

The mating parts of a seam should also use similar or compatible metals from the same group to reduce galvanic action in the presence of moisture. A table of the grouping of appropriate metals for shielding is shown below in Table 2.1. There are some overlaps because metals in adjacent groups can also be used.

Table 2.1: Grouping of metals for galvanic compatibility [6].

\begin{tabular}{|c|c|c|c|}
\hline Group 1 & Group 2 & Group 3 & Group 4 \\
\hline Magnesium & Aluminum & Cadmium Plating & Brass \\
\hline Magnesium Alloys & Aluminum Alloys & Carbon Steel & Stainless Steel \\
\hline Aluminum & Zinc \& Zinc Plating & Iron & Beryllium Copper \\
\hline Aluminum Alloys & Chromium Plating & Nickel \& Nickel Plating & Copper \& Copper Alloys \\
\hline Zinc \& Zinc Plating & Cadmium Plating & Tin \& Tin Plating & Nickel/Copper Alloys \\
\hline \multirow[t]{7}{*}{ Chromium Plating } & Carbon Steel & Tin/Lead Solder & Monel \\
\hline & Iron & Brass & Silver \\
\hline & $\begin{array}{l}\text { Nickel \& Nickel } \\
\text { Plating }\end{array}$ & Stainless Steel & \\
\hline & Tin \& Tin Plating & Beryllium Copper & \\
\hline & Tin/Lead Solder & $\begin{array}{l}\text { Copper \& Copper } \\
\text { Alloys }\end{array}$ & \\
\hline & & Nickel/Copper Alloys & \\
\hline & & Monel & \\
\hline
\end{tabular}




\section{Chapter 3}

\section{Door Assembly Design}

\subsection{Shield Design}

The shielding theory shown thus far indicates that metals would be the ideal choice for the construction of a door and frame. There are a few metals and alloys available on the market that are suitable for this task. Aluminum and stainless steel are the two commonly used metals in door construction [7]. Stainless steel is an alloy, which is commonly composed of iron, chromium, nickel, manganese, and copper [8], [9]. Aluminum has the advantage of being lighter with a density of roughly one-third of stainless steel [8], [9]. This results in stainless steel being stronger and harder to work with compared to aluminum. This also makes it harder to form but is less likely to bend and lose its form compared to aluminum [7]. When it comes to welding, stainless steel is a lot easier to work with. In the case of corrosion, both metals perform well. This is significant because corrosion increases surface impedance and results in lower shielding effectiveness. In stainless steel, chromium is added to provide corrosion resistance [8], [9]. Aluminum has a naturally occurring passivation layer of aluminum oxide, which prevents corrosion [8]. The biggest factor in a material's shielding effectiveness is the conductivity and this is where aluminum is a much better choice. Aluminum is roughly 34 times more conductive than stainless steel [7], [9]. This represents a significant improvement in overall reflection and absorption. Material cost is a big factor in the design process. Although aluminum is $1 / 3$ the density of stainless steel, its price per kilogram is almost 20 percent higher [10]. 


\subsection{Gaskets}

The seams between the door and the frame need to be shielded to prevent radiation as noted from Section 2.8. The choice of materials for the gaskets needs to be compatible with the metal of choice for the door and frame to prevent galvanic action. Two types of gaskets can be used in this application. The first option is a metal finger stock and the second is a metalized fabric over polyurethane foam [11].

\subsection{Metal Finger Stock}

Metal finger stock is the best choice when mechanical durability is required. They are robust in withstanding lateral and perpendicular shearing forces [12]. The two metals commonly used in finger stock are beryllium plated copper and stainless steel [11], [12].

Copper is soft and ductile with a high conductivity which is why it is widely used in electronic circuits. Alloying copper with other metals has the advantage of increasing its physical properties. In the case of beryllium, it increases the hardness of copper. This results in beryllium-copper exhibiting the mechanical properties of high-strength steel [12]. A small amount of nickel is also added roughly 0.2 percent minimum, which increases corrosion resistance. Beryllium copper can be found in Group 3 in Table 2.1, which makes it compatible with aluminum and stainless steel. The process of platting copper with beryllium reduces its conductivity by roughly 75 percent at a temperature of 20 degrees Celsius [11]. This results in diminished shielding effectiveness when compared to pure copper. Even with this reduction, beryllium copper finger stock is rated between $70 \mathrm{~dB}$ 
and $110 \mathrm{~dB}$ of shielding over a broad spectrum [11]. This has resulted in it being the industry standard and is widely used in shielding enclosures.

Stainless steel finger stock is an economical alternative to beryllium copper when shielding effectiveness requirements are low. The lower shielding effectiveness is a result of stainless steel's poor conductivity [11], [12].

Both finger stock types are offered in a variety of shapes and sizes as shown in Fig. 3.1. They also come in a variety of mounting options, which include stick-on, clip-on, snap-on, and hook and stick-on.

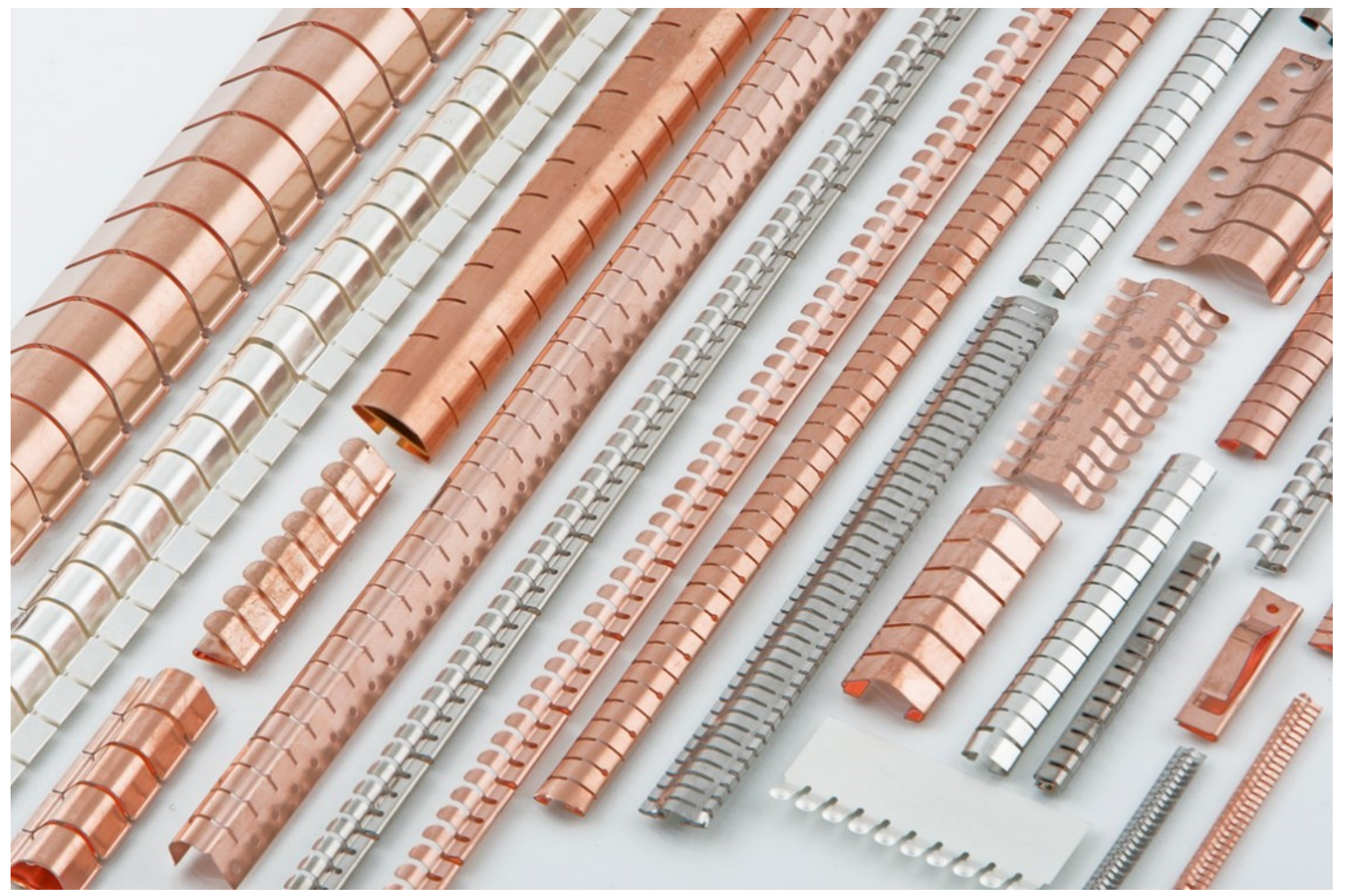

Figure 3.1: Metal fingerstock models [13]. 
To ensure the best shielding performance of a gasket, its size must be chosen accordingly to occupy the minimum and maximum gaps across a seam. The difference in gap spacing is generally the result of fabrication tolerances, misalignment of surfaces, or irregular surfaces [11]. The appropriate size should compress to 25 percent at minimum compression and 50 percent at maximum compression [11]. Examples of compression levels are shown in Fig. 3.2.

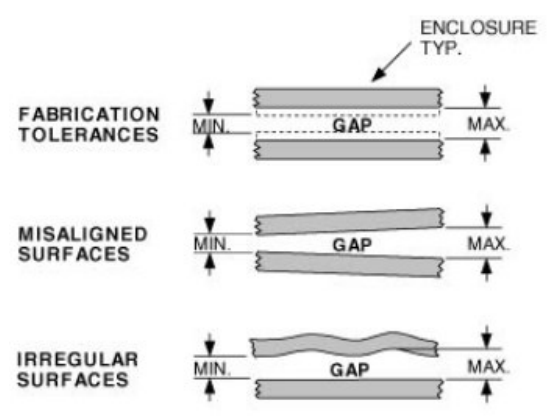

OPERATING RANGE = MAX. GAP - MIN. GAP
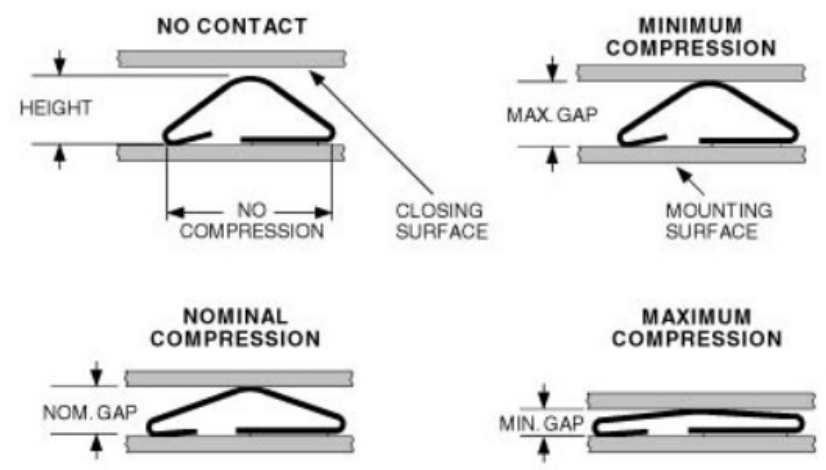

Figure 3.2: Alignment of surfaces and compression levels [11].

\subsection{Metalized Fabric Gaskets}

Metalized fabrics over foam gaskets are a cost-effective alternative to finger stock in shielding applications [12], [14]. The advances in plating and the availability of UL 94V-0 flame-rated materials have made fabric over foam gaskets increasingly popular [14]. The metalized fabrics come in a variety of blends, which include nickel-copper, nickel-silver, and stainless steel. Nickle-copper blend is the most popular due to its galvanic compatibility. As shown in Table 2.1 for galvanic compatibility, nickel-copper is compatible with groups 1 through 4. 
The foam used in the gaskets is generally polyurethane foam, which allows for flexibility and conformability [11]. Polyurethane foam gaskets are rated for use in the range of -40 to 90 degrees Celsius [11]. Other foams are available on the market, such as silicone, which is rated up to 220 degrees Celsius depending on the manufacturer [12], [14]. There are a variety of shapes and profiles for fabric over foam gaskets available on the market as shown in Fig. 3.3. Some manufacturers offer customizable gasket which are fabricated from CAD drawings like the ones shown at the bottom right of Fig. 3.3. The various shapes and sizes make these gaskets the ideal choice for an enclosure where weight and space are limited.

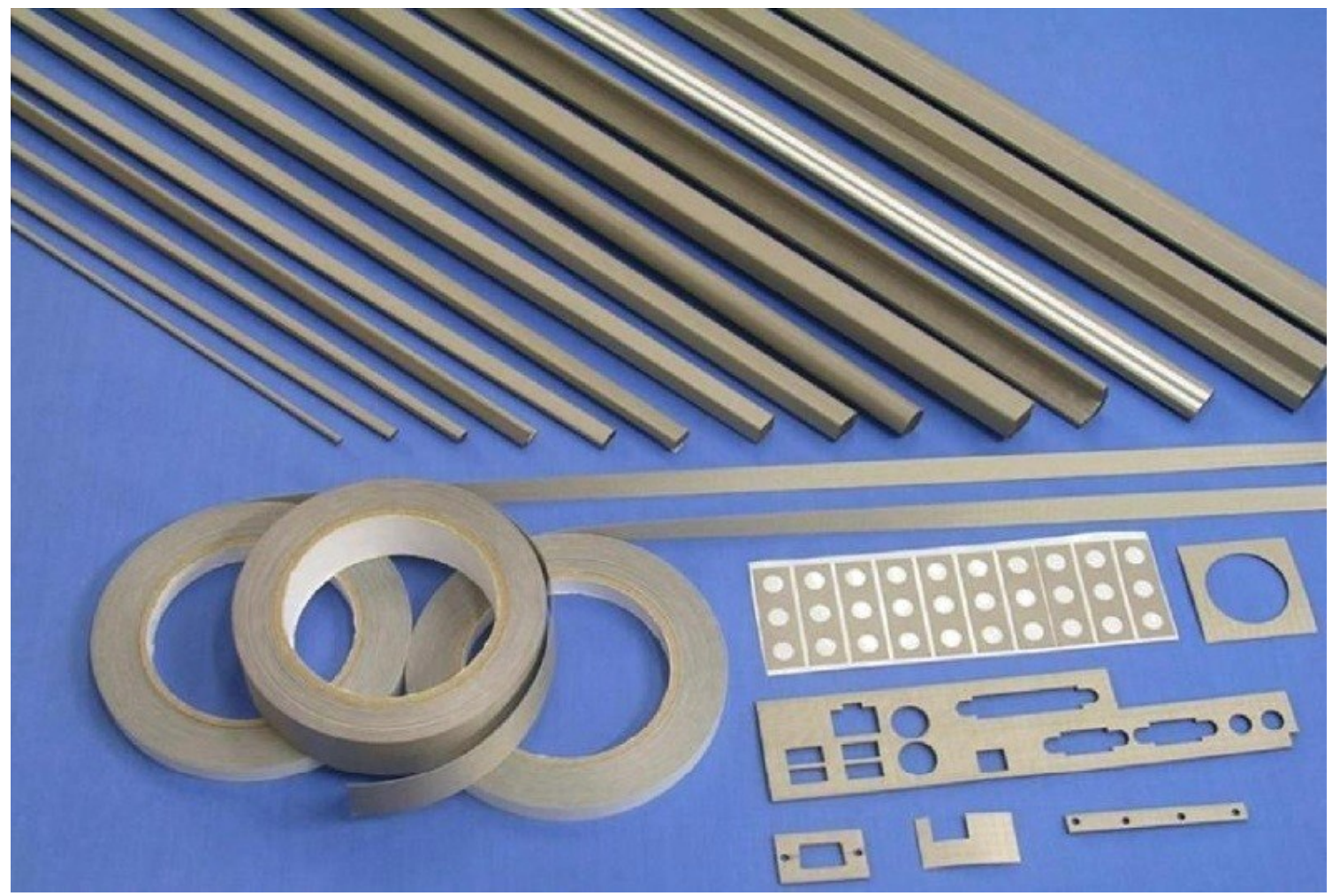

Figure 3.3: Metalized fabric over foam gaskets [15]. 
Fabric over foam gaskets are excellent in applications with low compression force [12], [14]. The foam provides a larger surface area contact, which reduces the transfer impedance and provides higher shielding effectiveness. Most foam gaskets on the market offer low compression set [14], [16]. Compression set measures the amount of permanent deformation of a material after recovery when exposed to a specific compression at a given temperature [17]. A three percent compression set means that 97 percent of the material regains its original shape. This is important for enclosures with access doors or panels as it provides snug contact around bends and corners.

A comparison of shielding effectiveness between fabric over foam gasket and finger stock as a function of compression is shown in Fig. 3.4. The shape of the foam gasket is D-shape, which is the same as the finger stock. The results show fabric over foam outperforms finger stock in applications requiring low compression.

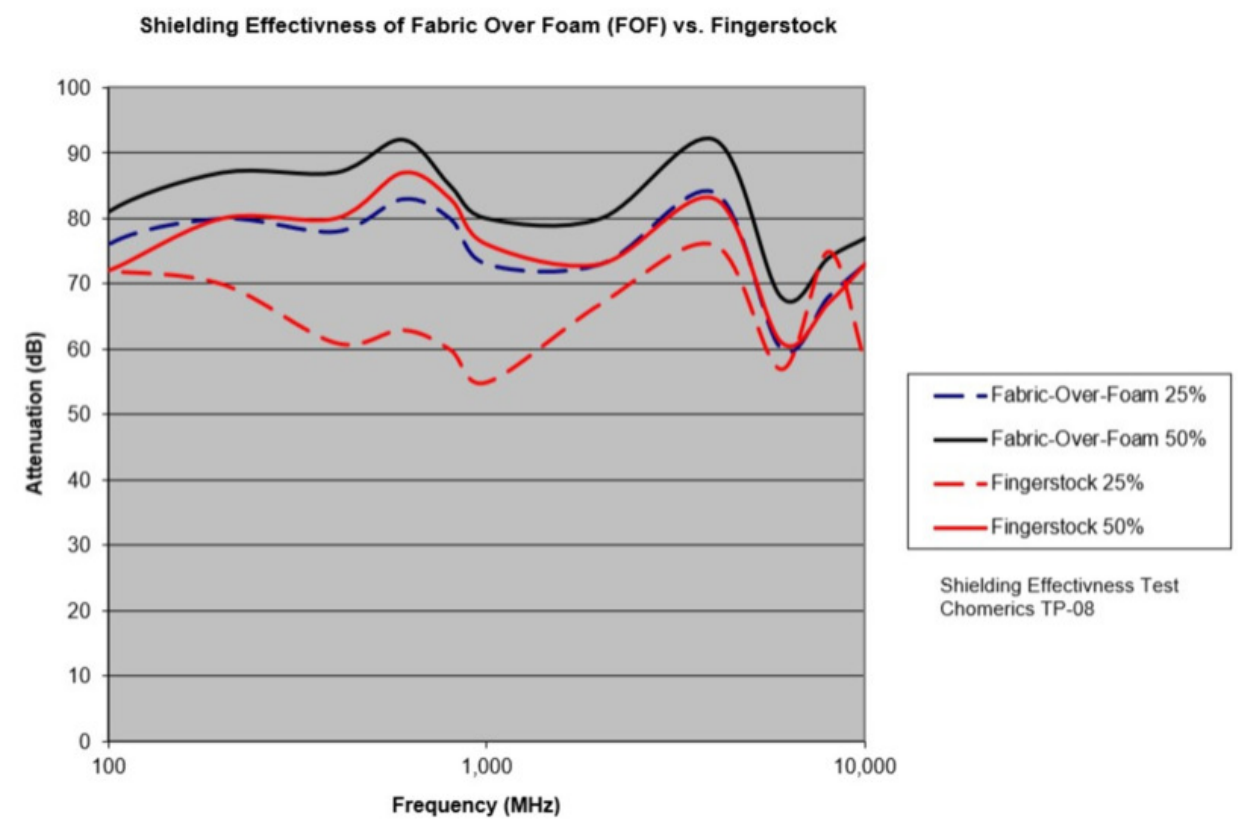

Figure 3.4: Gasket SE for $25 \%$ and $50 \%$ compression [14]. 
There are a few advantages of fabric gaskets compared to finger stock. Foam gaskets are easier to install and provide moisture sealing when compared to finger stock [14]. The slots in finger stock are designed to take up gap tolerances and reduce the force need to deflect across a seam. As frequencies increase, the leakage through these slots will increase [14].

\subsection{Door Assembly Design}

The initial design of the door and frame assembly present by Ambico is shown below in Fig. 3.5. Stainless steel was chosen for the construction of the door and the frame due to a variety of benefits when compared to aluminum. These include the lower price point, durability, and sturdiness.

Sixteen gauge (1.59 mm thick) stainless steel was chosen for the door panels and frame. This thickness is easily formable and provides sufficient shielding. The top and bottom end channels of the door also use the same thickness. Looking at Fig. 2.5, the absorption alone is well beyond $175 \mathrm{~dB}$ at $1 \mathrm{GHz}$ for a thickness of $1.59 \mathrm{~mm}$. The door handles and threshold beneath the door are made of stainless steel as well for galvanic compatibility. A detailed view of the threshold is shown in Fig 5.6 in Chapter 5.

A honeycomb core is used in the interior of the panels to enhance the structural integrity and significantly reduce the weight of the door. The core is made of cardboard that is hardened by special resins to make it heavy-duty and durable [18]. It is mostly used for interior openings and is generally fire-rated for up to 3 hours. The core is shown below in Fig. 3.5. 
The design uses both types of gaskets to seal the seams. The gaskets placements are shown in Fig 5.7 in Chapter 5.
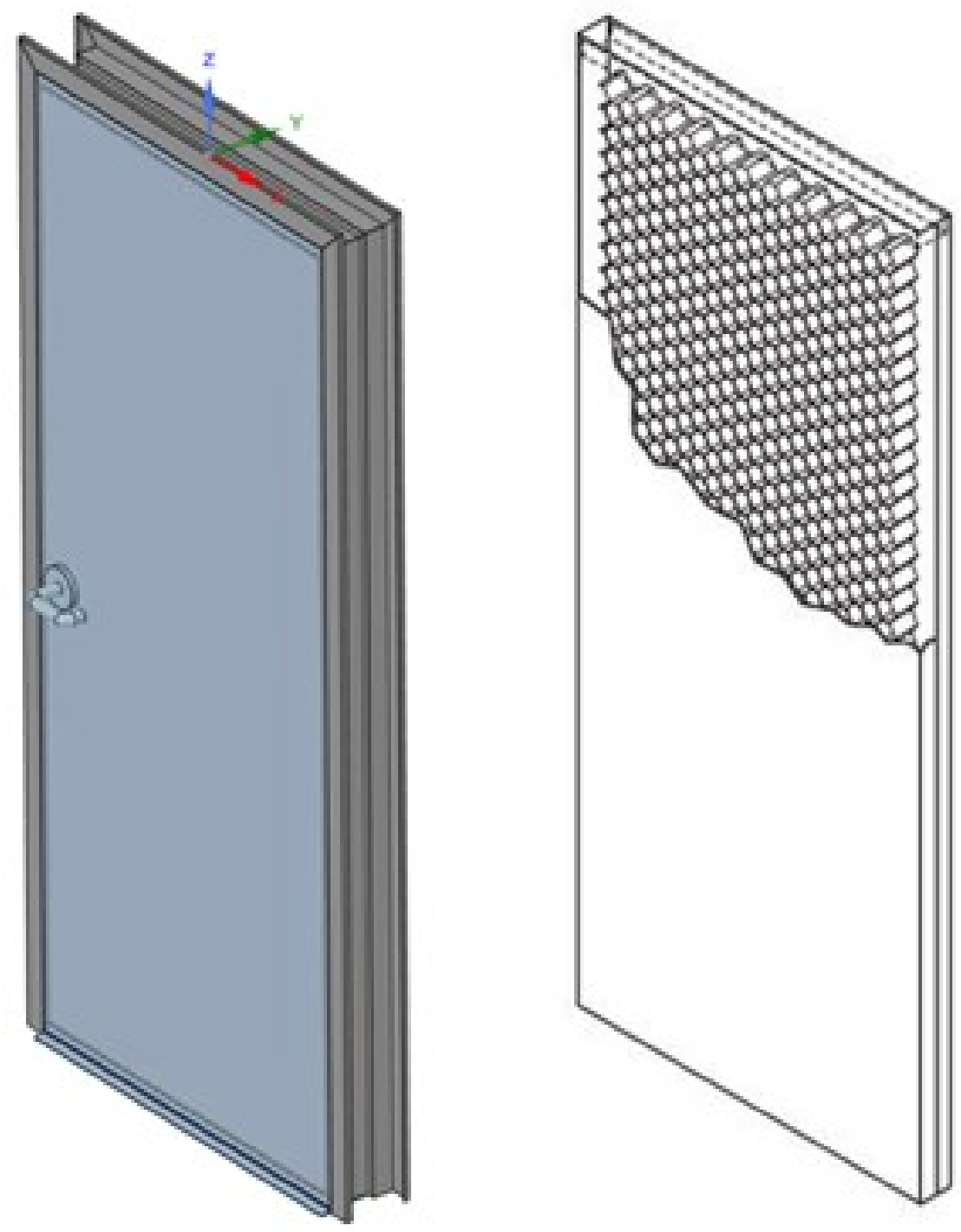

Figure 3.5: 3D rendering of door and frame assembly with honeycomb core [18]. 


\section{Chapter 4}

\section{Electromagnetic Simulations}

\subsection{Ansys EDT Simulations}

Ansys Electronic Desktop (EDT) uses a 3D full-wave frequency domain EM filed solver using the finite element method (FEM). With EDT the S, Y, and Z parameters of components can be extracted. It also allows full 3D EM wave visualization for near and far filed.

The simulation environment for this work in Ansys EDT consists of two pyramidal horn antennas and the assembly contained in a radiation boundary as shown in Fig. 4.1. The assembly is placed between the transmitting and receiving antenna to measure its shielding effectiveness. The purpose of the radiation boundary is to prevent leakage around the edges of the structure and reduce the computational power required for the simulations. With this setup, leakage is only possible through the seams.

The materials used in the simulations are set according to the design. Stainless steel for all the parts of the assembly except the gaskets. Beryllium copper and nickel-copper materials were created in the materials library in EDT for the gaskets.

Frequencies between $1 \mathrm{GHz}$ and $3.2 \mathrm{GHz}$ at intervals of $200 \mathrm{MHz}$ were used for the simulations. This span was sufficient to obtain theoretical performances while keeping simulation times reasonable. The horn antennas were modified accordingly for the ranges of frequencies used. 


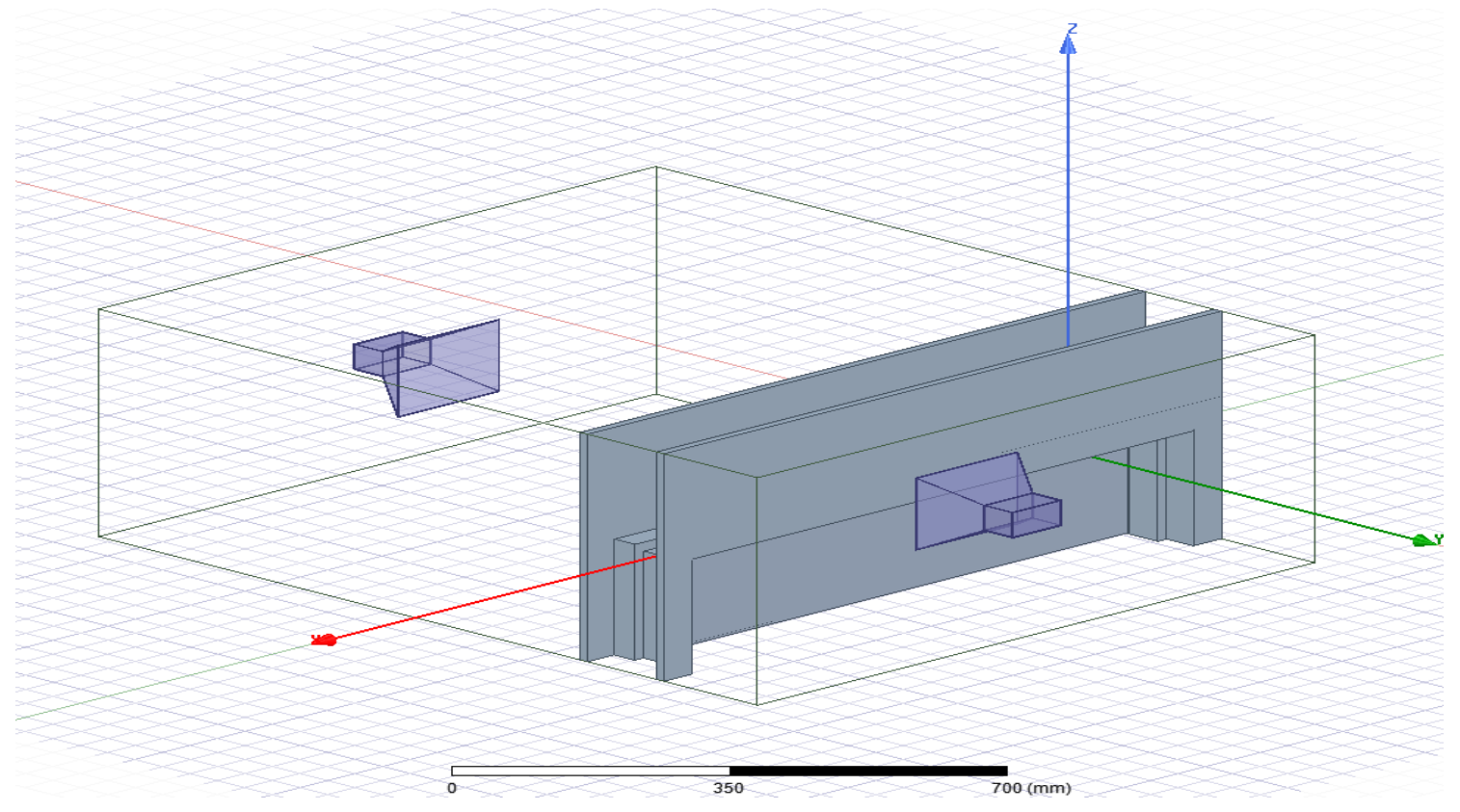

Figure 4.1: Simulation environment with top seam.

\subsection{Top Seam SE}

The structure shown in Fig. 4.1 is a modification of the door and frame assembly as it mainly exposes the top seam for SE measurements. This modification ensures that leakage of any significance is contained to the top seam. It also reduces the simulation time since the size of the structure is smaller and the radiation boundary has a lower volume.

A reference measurement of the transmission between the two antennas was first obtained before the SE of the top seam. The simulation results of the SE of the top seam are shown below in Fig. 4.2. The results show that the top seam has roughly a $10 \mathrm{~dB}$ dropoff in $\mathrm{SE}$ at $2 \mathrm{GHz}$ compared to the rest of the spectrum. 


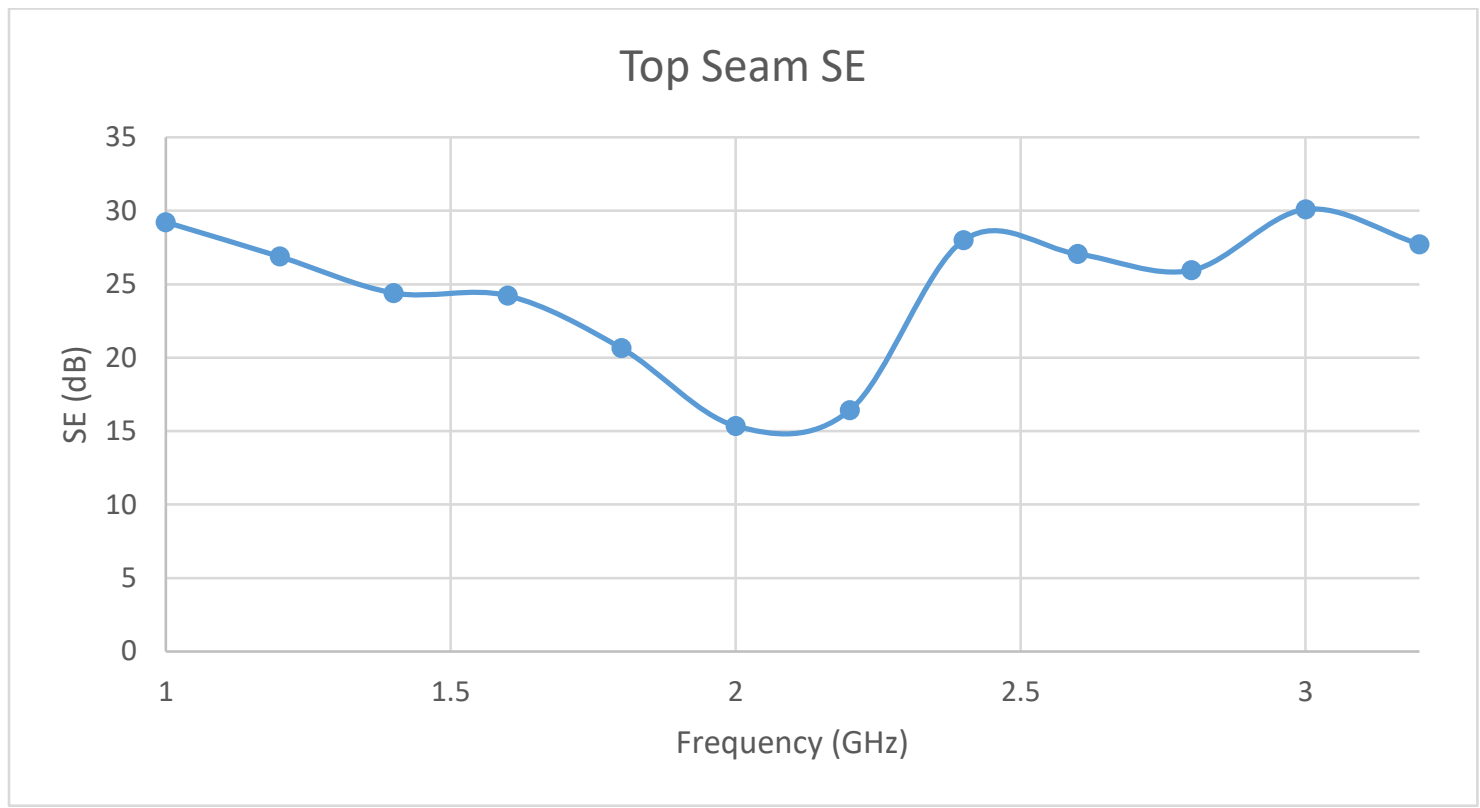

Figure 4.2: Top seam SE.

\subsection{NiCu Fabric Gasket}

Nickel copper fabric over foam gasket generally have a surface resistance $\left(R_{S}<0.02\right.$ Ohms/square) with a fabric thickness $(t=0.08 \mathrm{~mm})$. The fabric was modeled as a material with a bulk conductivity given by

$$
\sigma_{N i C u}=\frac{1}{R_{S} \times t}=625 \mathrm{KS} / \mathrm{m}
$$

This is possible since the skin depth is approximately $20.1 \mu \mathrm{m}$ at $1 \mathrm{GHz}$, which is approximately $1 / 4$ of the thickness of the fabric. Therefore the polyurethane foam inside the fabric does not need to be modeled since very little energy gets to it. 
The D and C-Fold profiles shown in Fig. 3.3 are suitable gaskets to cover the seams. The cfold profile was chosen for this design because of its flexibility. The 3D model of the gasket is shown below in Fig. 4.3. The location of the gasket was varied between the front and end of the top of the door as shown in Fig. 4.4 to find the effects of gasket locations on SE.

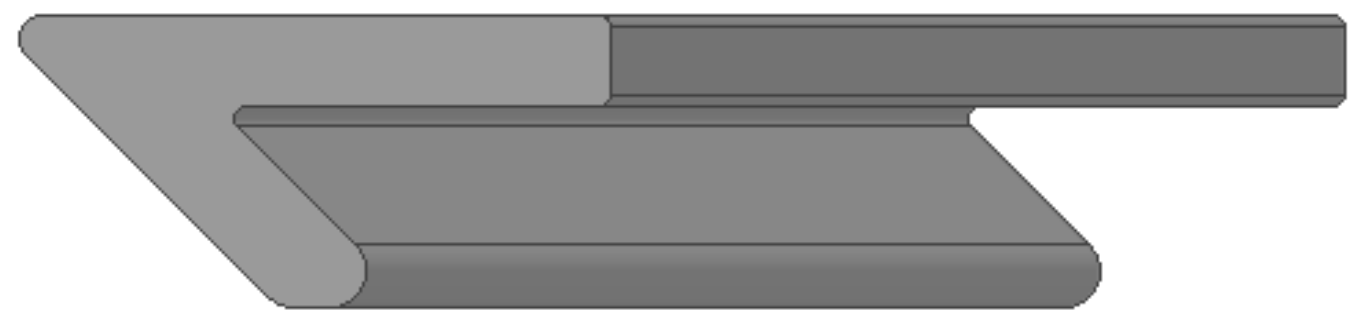

Figure 4.3: NiCu FOF 3D gasket model.
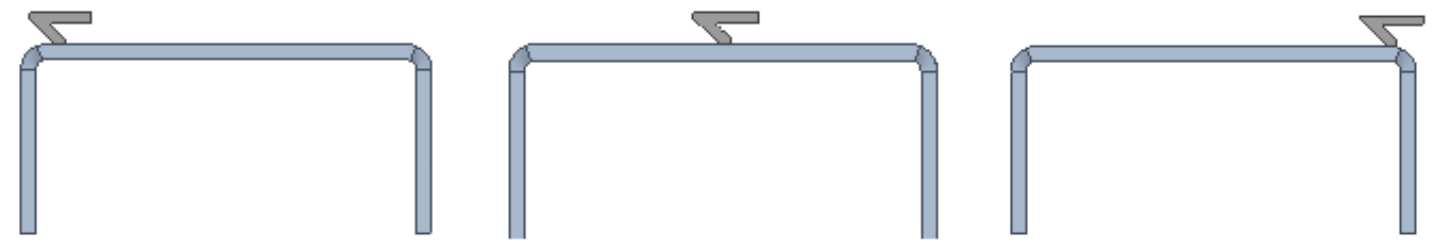

Figure 4.4: NiCu FOF gasket placement at the top seam.

The results for the simulation are shown below in Fig. 4.5. The results show a trend of degradation in SE when the gasket is moved further back. The middle placement of the gasket has the worst performance when compared to the others. It is especially noticeable at $2.6 \mathrm{GHz}$ where the $\mathrm{SE}$ is down by roughly $28 \mathrm{~dB}$ compared to front gasket placement. This drop can be explained by looking at the distance between the gasket 
contact and the front of the door. The distance between them is roughly $22.2 \mathrm{~mm}$ which is close to $28.8 \mathrm{~mm}, \mathrm{a} 1 / 4$ of a wavelength at $2.6 \mathrm{GHz}$. This is significant since microwave theory shows that a short is converted to an open with a $1 / 4$ wavelength line. It has been shown that the length of a $1 / 4$ wavelength line can be reduced with capacitive loading. In this case, the capacitance is between the end channel and the front and back panels of the door. In this sense, the gasket's transfer impedance has been greatly increased resulting in poor SE.

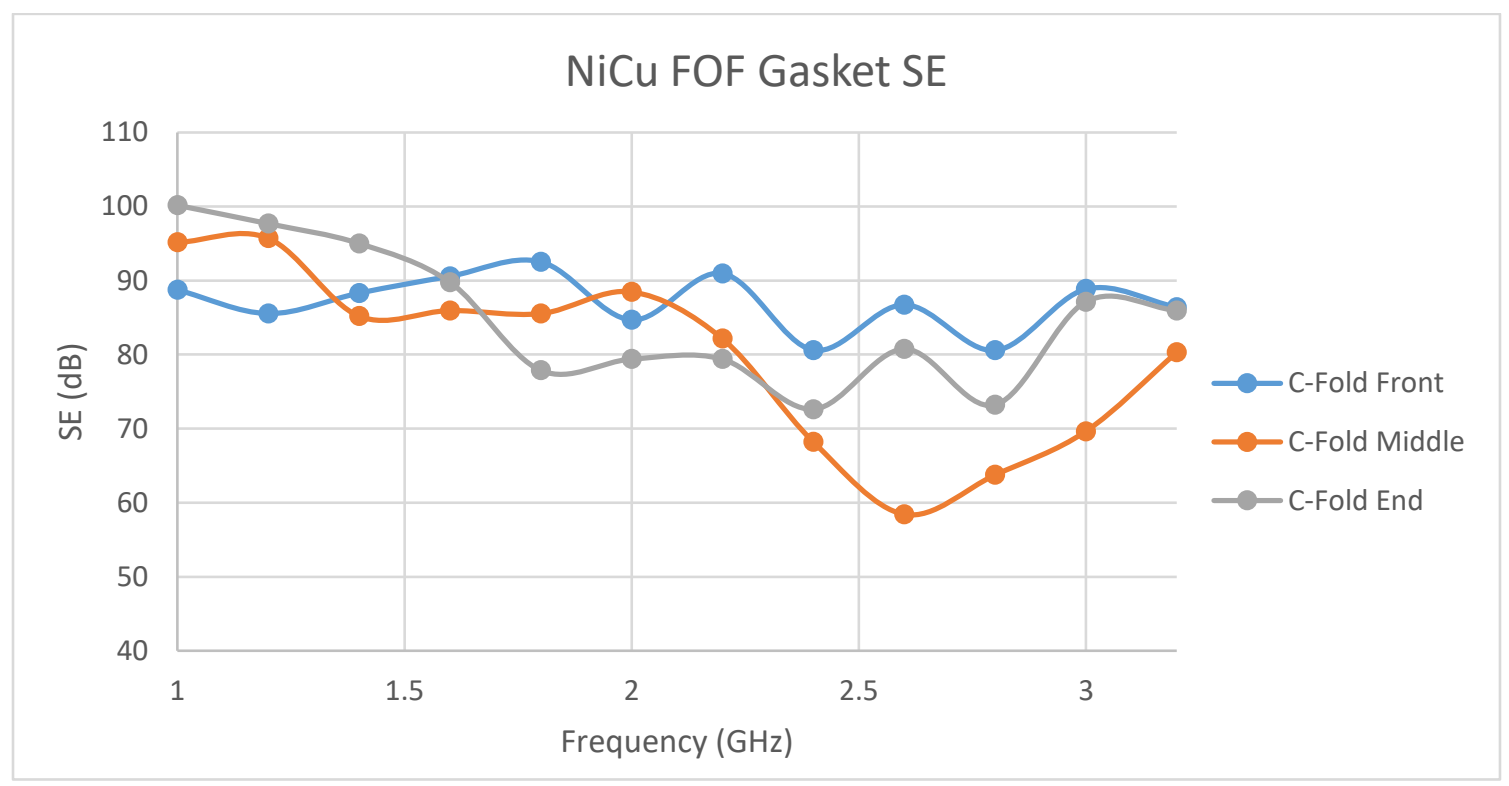

Figure 4.5: SE of NiCu FOF gasket for front, middle and back location.

A second model of the gasket was used to simulate the effect of compression would have on the SE of the gasket. This comparison looks at the difference when both gaskets are at the front of the seam. The side profile of the gasket is shown below in Fig. 4.6 and the simulation results in Fig. 4.7 . 
These results are unexpected since the uncompressed gasket has a slightly better performance. The uncompressed gasket has an average SE of $87 \mathrm{~dB}$ while the compressed case is $83.4 \mathrm{~dB}$. In reality, compression creates a larger surface contact which tends to reduce the surface impedance as shown in Fig. 3.4 resulting in improved SE.

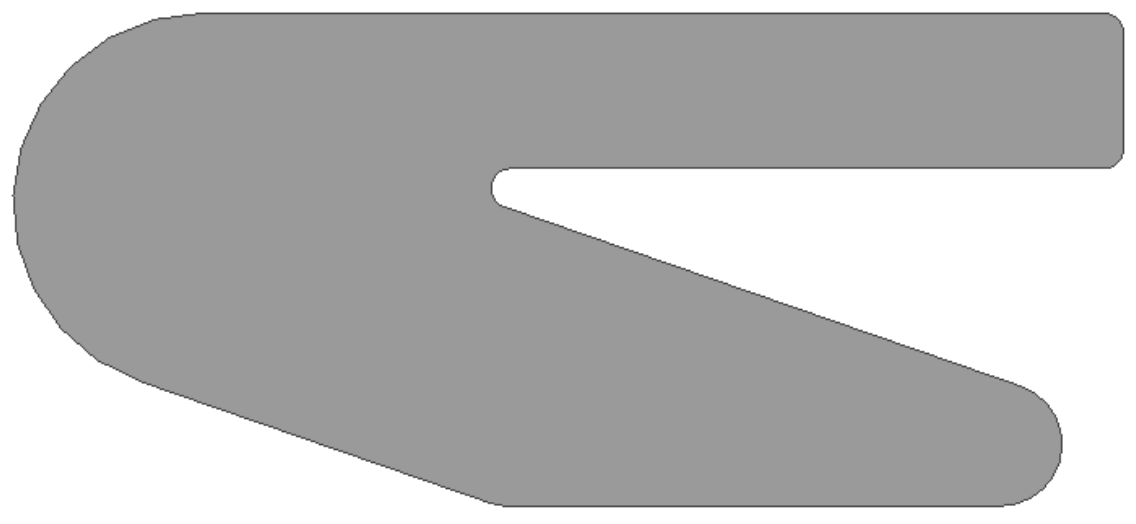

Figure 4.6: Compressed NiCu FOF gasket 3D model.

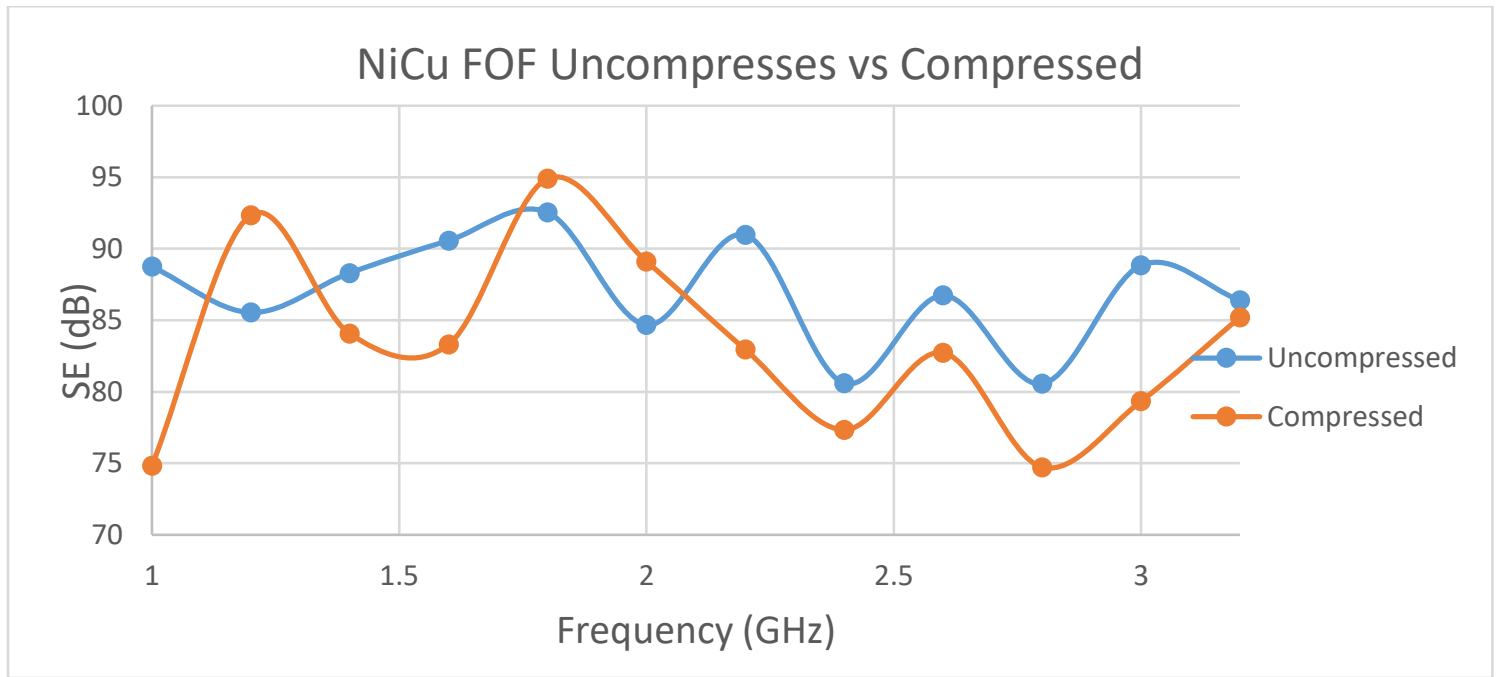

Figure 4.7: Compression comparison of BeCu fingerstock. 


\subsection{BeCu Fingerstock}

Beryllium copper fingerstock is generally rated with an electrical conductivity (\% IACS) of 22 to 25 . This value represents a percentage of the conductivity of copper, which is 58 $\mathrm{MS} / \mathrm{m}$. For the simulations, BeCu was modeled using a material with an average \% IACS of 23.5 which translate to a bulk conductivity $\sigma_{B e C u}=13.63 \mathrm{MS} / \mathrm{m}$

Two different 3D models of the fingerstock were used to model the effects of gasket compression. The profile of the two models is shown below in Fig 4.8. The first model represents little compression while the second provides a larger surface area contact to represent compression. Both gaskets have the same spacing of $0.8 \mathrm{~mm}$ between fingers as shown in the 3D view in Fig 4.8. In these simulations, both gasket models were placed at the front of the seam. The results of the simulations are shown below in Fig 4.9.

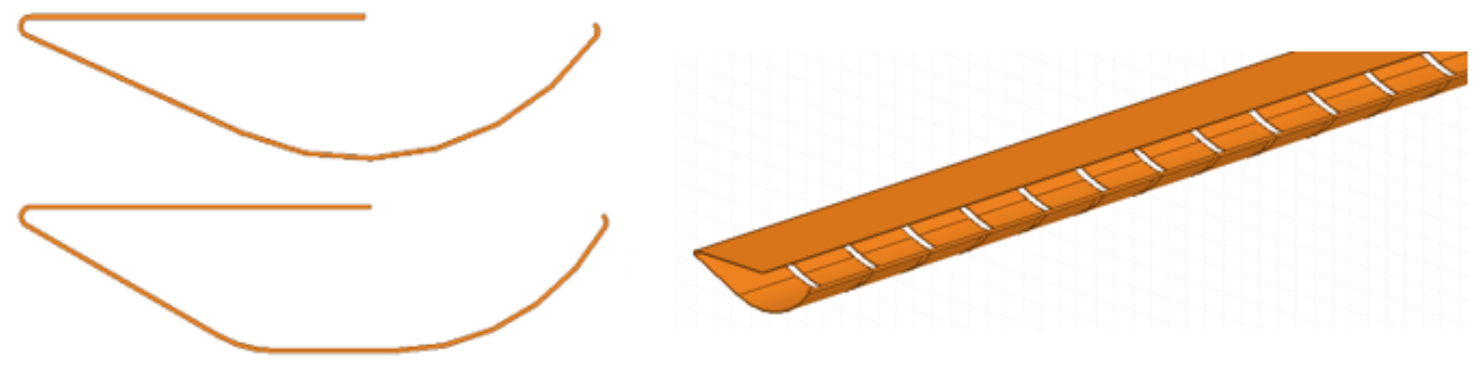

Figure 4.8: BeCu fingerstock 3D models.

Both models show a similar pattern in SE and as did the FOF case, the uncompressed gasket had a slightly better performance. The average SE for the uncompressed model is $81.1 \mathrm{~dB}$ and $78.4 \mathrm{~dB}$ for the compressed model. In both cases, the $\mathrm{SE}$ at $1.8 \mathrm{GHz}$ is greatly 
increased compared to the average. The same explanation of a $1 / 4$ wavelength distance used for the FOF case can be used here but, in this situation, it is the reverse. What looks like an open at the back of the seam is converted to a short at the front. The distance between the gasket contact and the back of the seam is approximately $38.7 \mathrm{~mm}$ which corresponds to a $1 / 4$ wavelength at $1.9 \mathrm{GHz}$.

A comparison between both materials when the gasket is located at the front of the seam is shown below in Fig 4.9. As expected, the NiCu fabric has a better performance compared to BeCu finger stock. Note again that both gaskets have their SE peak at 1.8 $\mathrm{GHz}$ due to gasket location. This phenomenon is more pronounced in the BeCu case due to its superior conductivity it provides a better short.

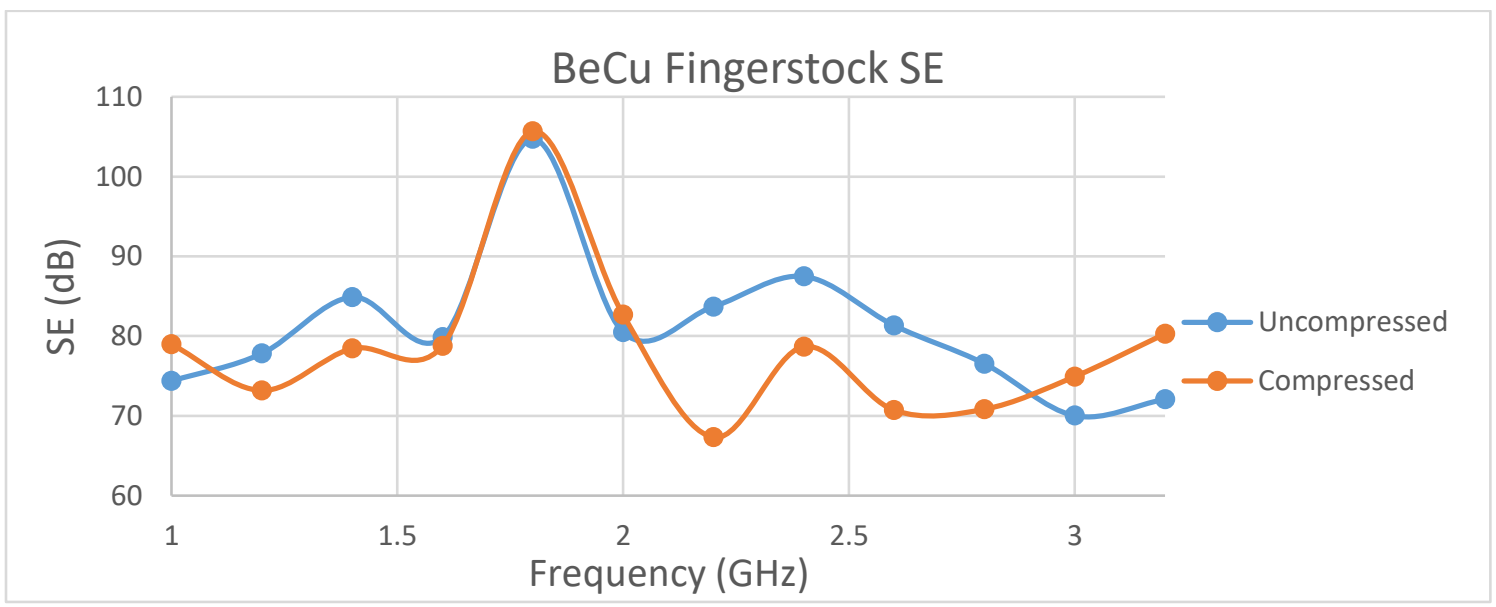

Figure 4.9: Compression comparison of $\mathrm{BeCu}$ fingerstock. 


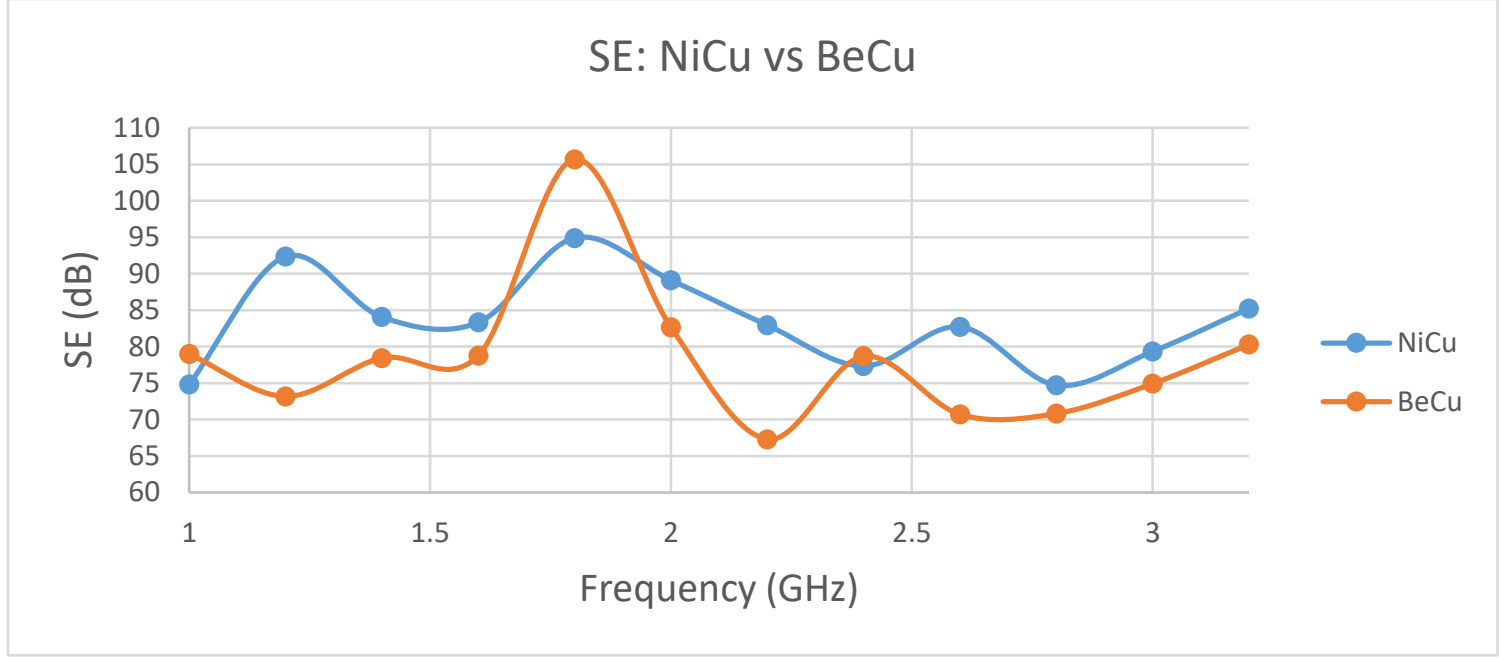

Figure 4.10: Comparison of BeCu and NiCu gaskets.

\section{5 $\quad \mathrm{BeCu}+\mathrm{NiCu}$ Gaskets}

This experiment looks at the performance when both types of gaskets are used at the same time. In the previous section that looked at the NiCu gasket, the results showed that placing the gasket at the front or the back of the seam had a similar performance. The middle placement was compromised in SE at $2.6 \mathrm{GHz}$. It would then be reasonable to look at the behavior of placing both gaskets at the front and back together.

Two simulations were performed with both gasket types. In the first case, the BeCu gasket was placed at the front and the $\mathrm{NiCu}$ at the back. The second case had the gaskets switched and both simulations used the compressed models of the gaskets. The simulation results are shown below in Fig 4.11 and the comparison of all four cases of gaskets used for sealing the top seam is shown in Fig 4.12.

Looking at the simulation results, the second case of $\mathrm{NiCu}$ followed by $\mathrm{BeCu}$ has better overall SE performance. The response of this combination behaves like a bandpass filter 
in the range of $1.6 \mathrm{GHz}$ to $2.2 \mathrm{GHz}$. Similarly, the combination of $\mathrm{BeCu}$ followed by $\mathrm{NiCu}$ has the same type of behavior in the range of $1.6 \mathrm{GHz}$ to $2 \mathrm{GHz}$ but beyond $2 \mathrm{GHz}$, the SE is on a $-25 \mathrm{~dB} / \mathrm{GHz}$ slope.

When comparing all four cases shown in Fig 4.12, NiCu placed at the front on its own has slightly better performance on average. Depending on the shielding application, these results show that a shielding response can be customized with gasket type and location at a seam.

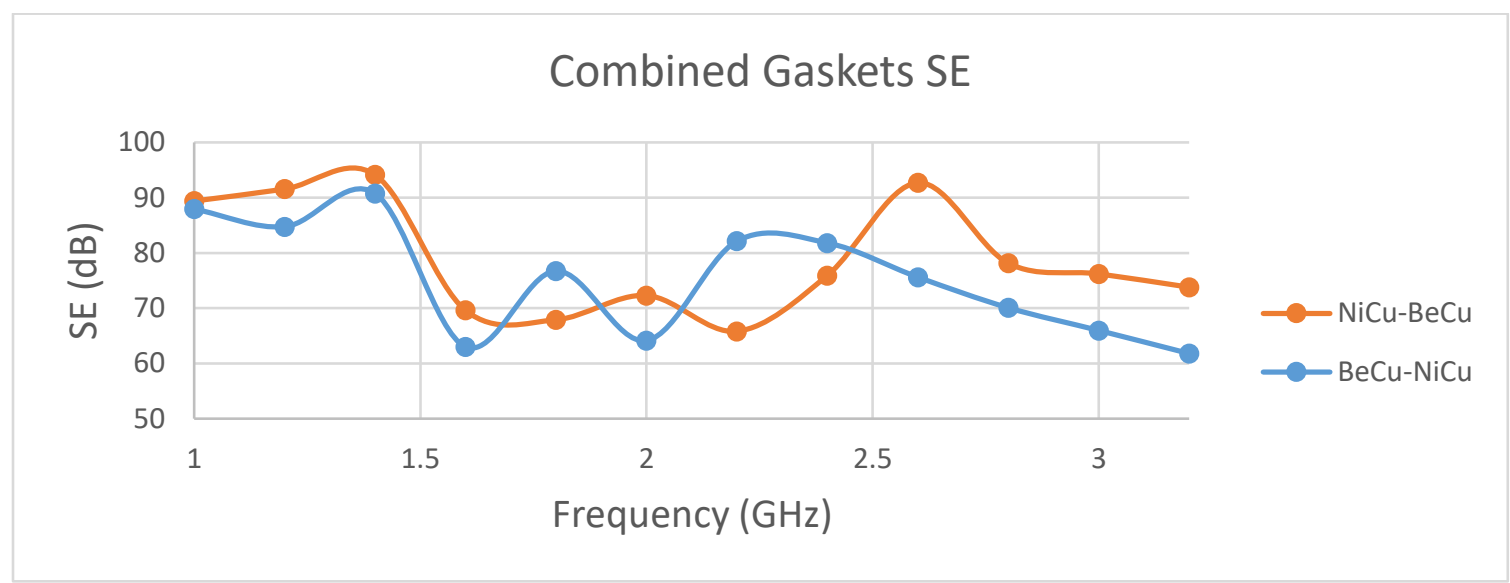

Figure 4.11: Comparison of $\mathrm{NiCu}-\mathrm{BeCu}$ and $\mathrm{BeCu}-\mathrm{NiCu}$ gasket placement. 


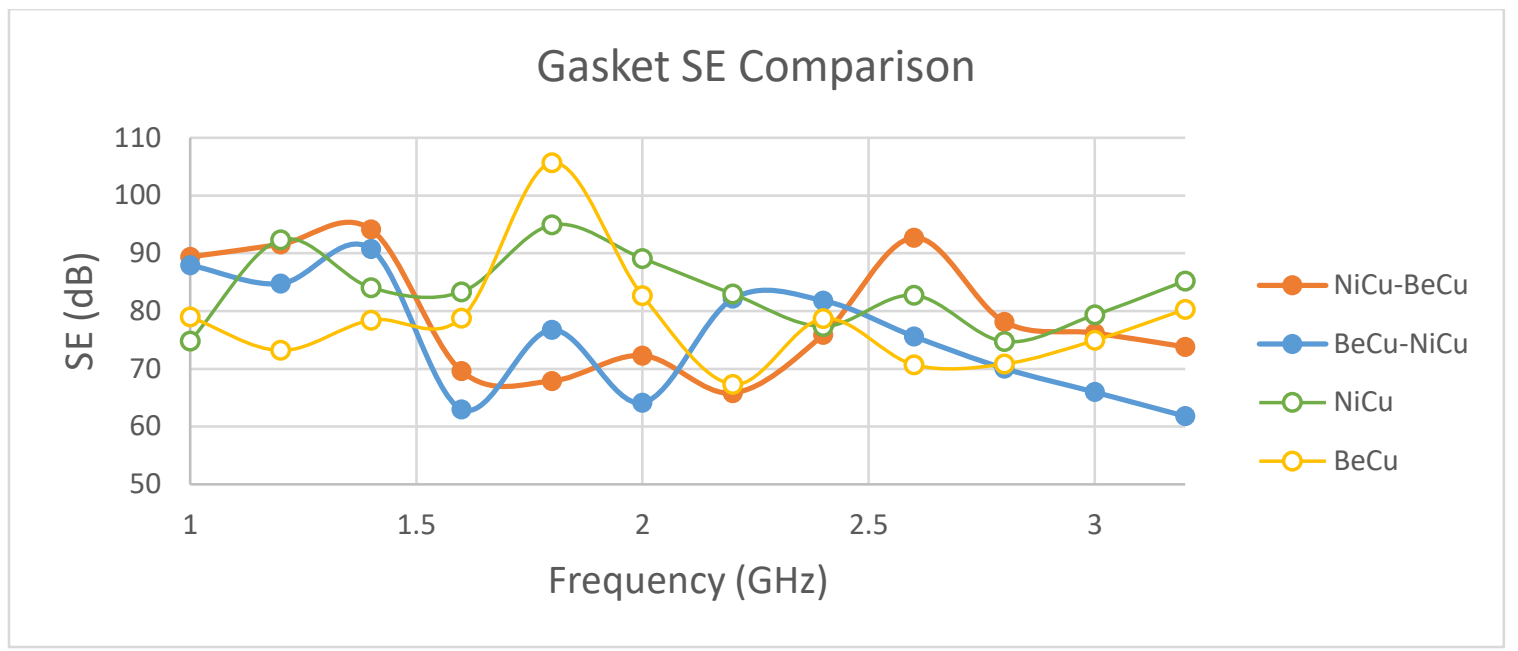

Figure 4.12: Comparison of all 4 cases of gasket configuration.

\subsection{Corner SE}

A separate 3D model of the assembly was created to test the corner of the door. The model has the corner centered at the horn antennas for maximum illumination as shown in Fig 4.13. The frame was stretched to the radiation boundary to prevent side leakage. In this way, the majority of the leakage is from the corner as part of the top and side seams are exposed.

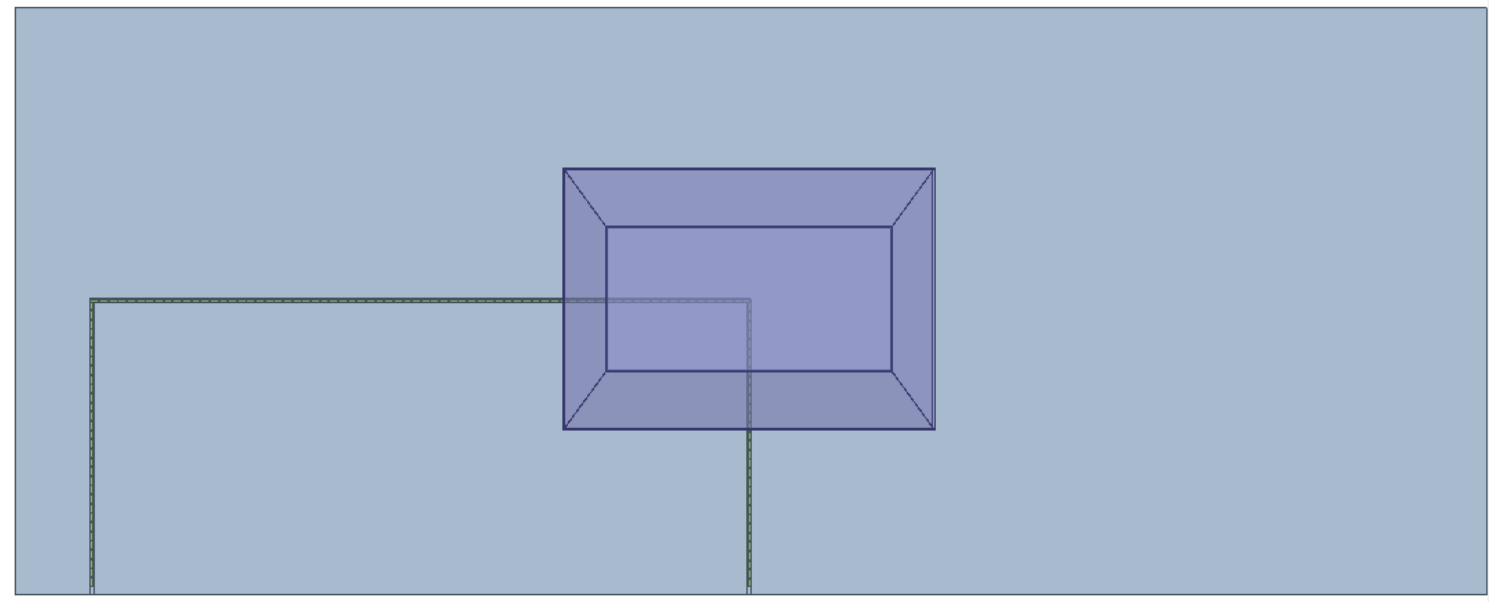

Figure 4.13: Corner illumination of the door frame. 
The SE of the corner including the SE of the top seam for comparison is shown below in Fig 4.14. The simulations show that the top seam has roughly $10 \mathrm{~dB}$ or greater leakage compared to the corner of the door. This again points to the radiation efficiency of long thin slits discussed previously in the shielding theory.

The SE performance of the corner including the top seam with both gaskets is shown in Fig 4.15. The configuration of the gaskets in both cases has $\mathrm{BeCu}$ followed by $\mathrm{NiCu}$. The results show similar SE patterns for the top and corner.

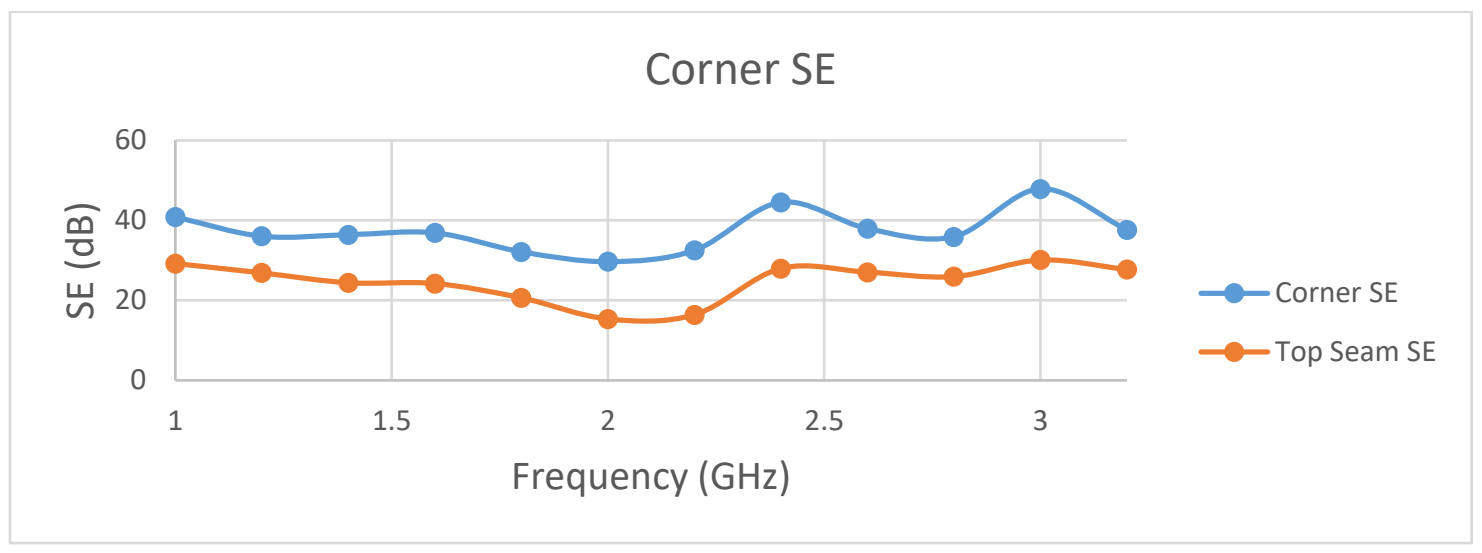

Figure 4.14: Comparison of SE of the corner and top seam.

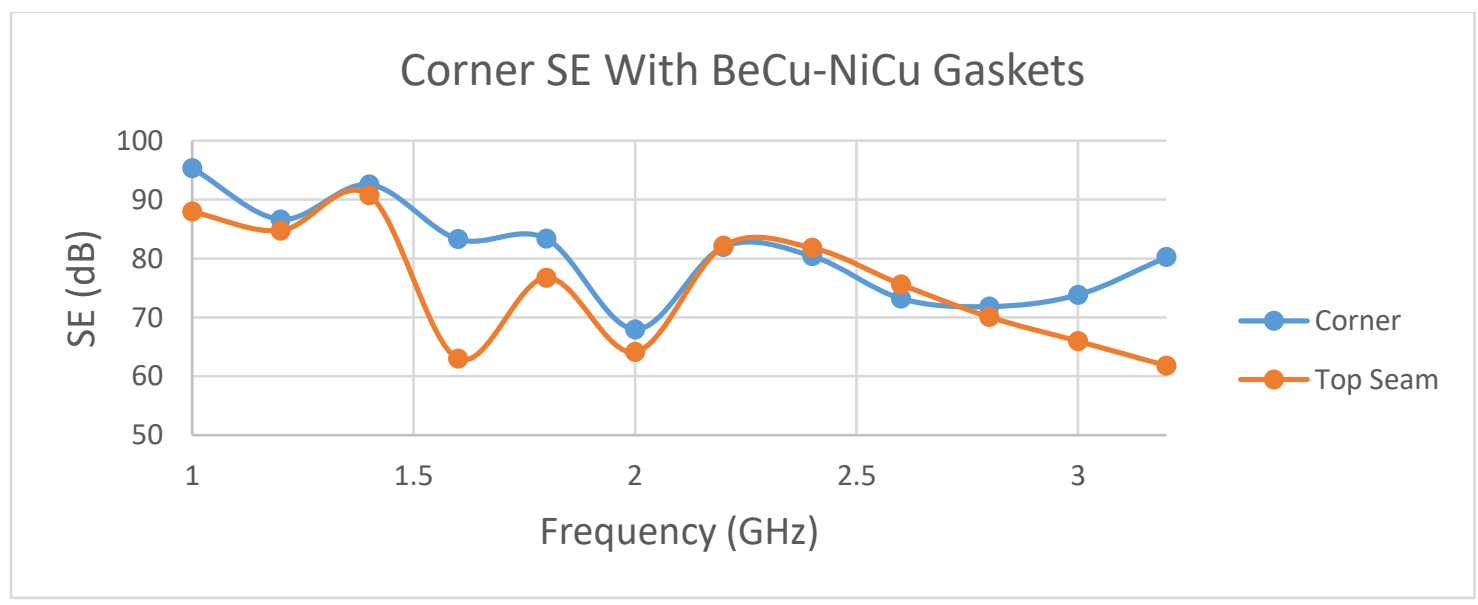

Figure 4.15: SE comparison of corner and top seam with same gasket configuration. 


\subsection{Conclusion}

Shielding effectiveness simulations were performed on the top seam as well as the corner between the top and right seam. The gaskets used in the simulations are $\mathrm{BeCu}$ fingerstock and $\mathrm{NiCu}$ fabric over foam gaskets.

The SE simulation results show that gasket location along the depth of the seam has an impact on the performance. This degree of freedom can be used to meet stricter requirements. The results also show that the use of multiple gaskets can be used to tailor the SE response at the seams. The simulations were useful in determining the trends between gasket configurations but not the expected SE performance since this was an Ideal environment. 


\section{Chapter 5}

\section{Radiated Testing}

\subsection{Anechoic Chamber Testing}

The miniaturized door and frame assembly design is shown below in Fig 5.1. The door, the frame, and the front panel were fabricated using stainless steel. The housing for the receiving antenna is made of galvanized steel. The purpose of choosing galvanized steel was to reduce the material cost while still maintaining galvanic compatibility. This is possible since galvanized steel is plain steel coated with zinc and is in the adjacent group to stainless steel in Table 1 for galvanic compatibility.

The detailed views of Section A-A in Fig 5.3 and Fig 5.4 show the difference between lock seam and fully welded edges for the two types of doors tested. The two mating parts of the lock seam are spot welled to prevent them from sliding. A honeycomb core is used in both doors as depicted in the figure. Note that both gasket types are in place at the side seam in the detailed view and are drawn uncompressed.

The top seam is shown in the detail in Fig 5.6 and the bottom seam in Fig 5.7. The gasket at the back of the door is added to reduce the impedance transfer between the door and the frame. The last gasket beneath the threshold is to improve contact between the threshold and the floor in case of misalignment. 


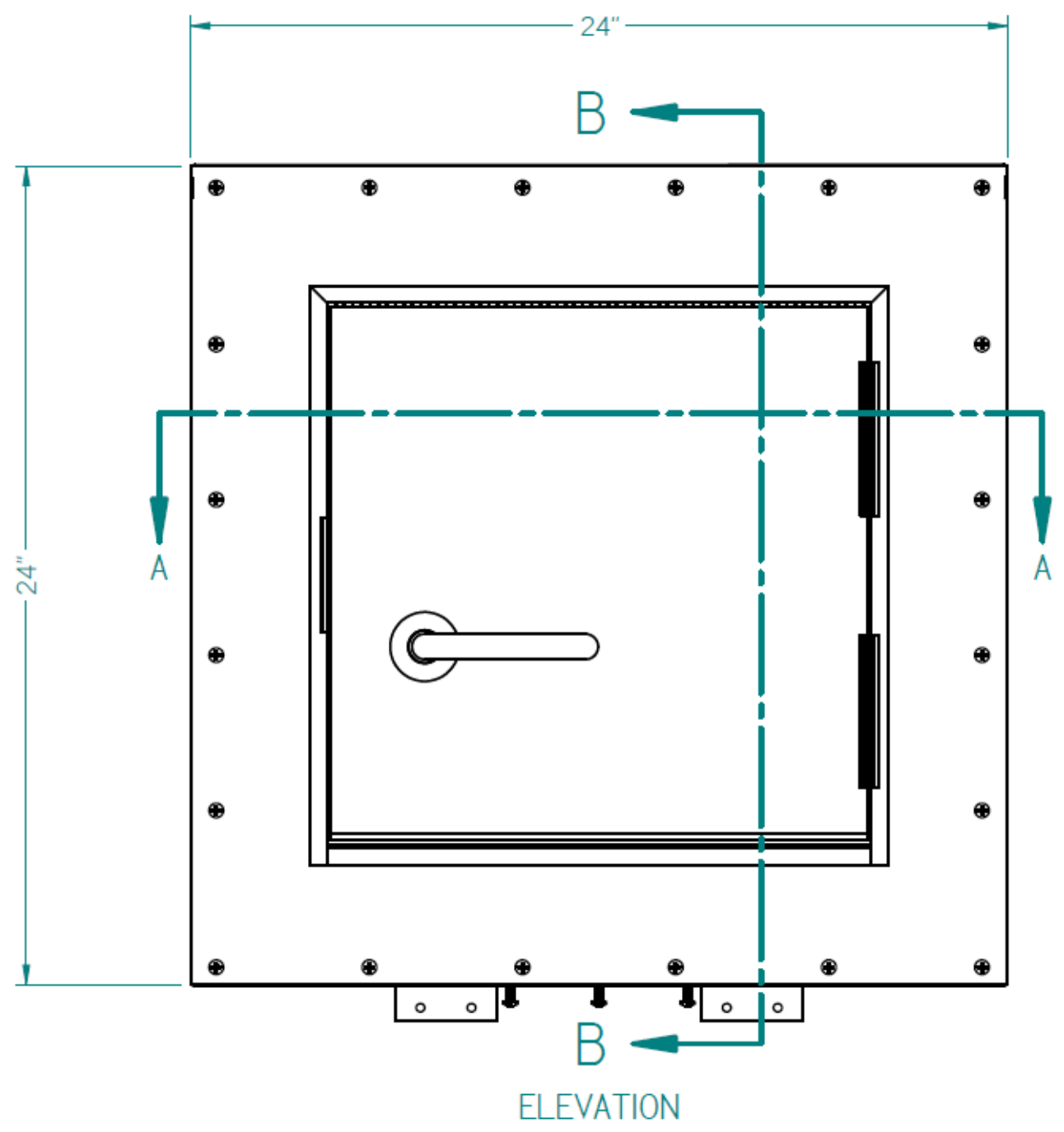

Figure 5.1: Miniaturized door and frame assembly. 


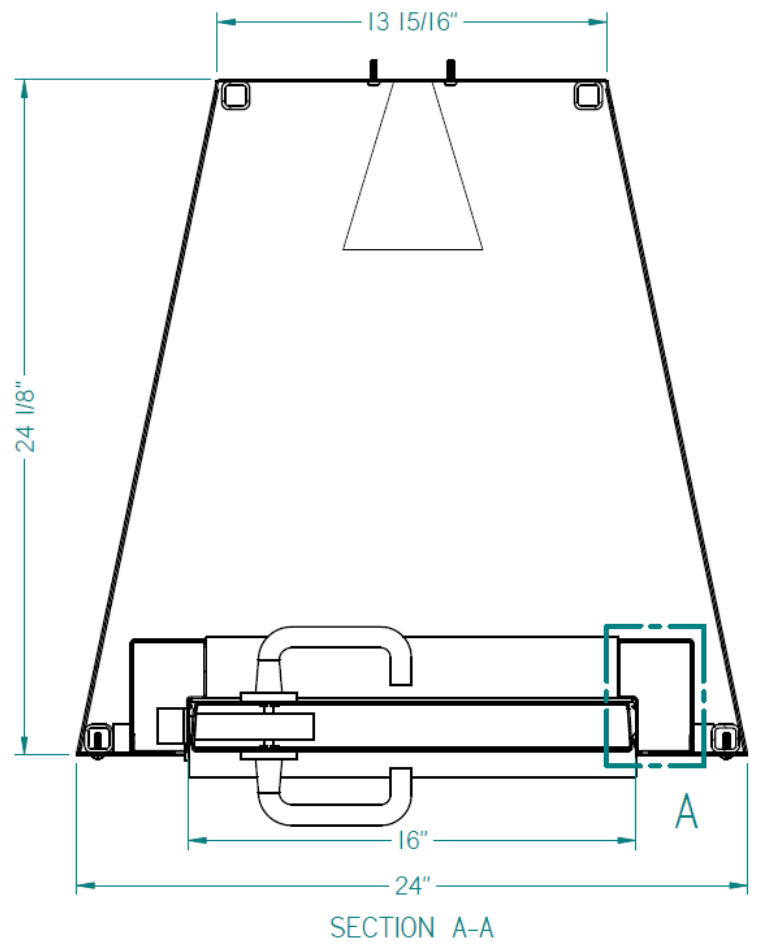

Figure 5.2: Section A-A of Miniaturized frame assembly.

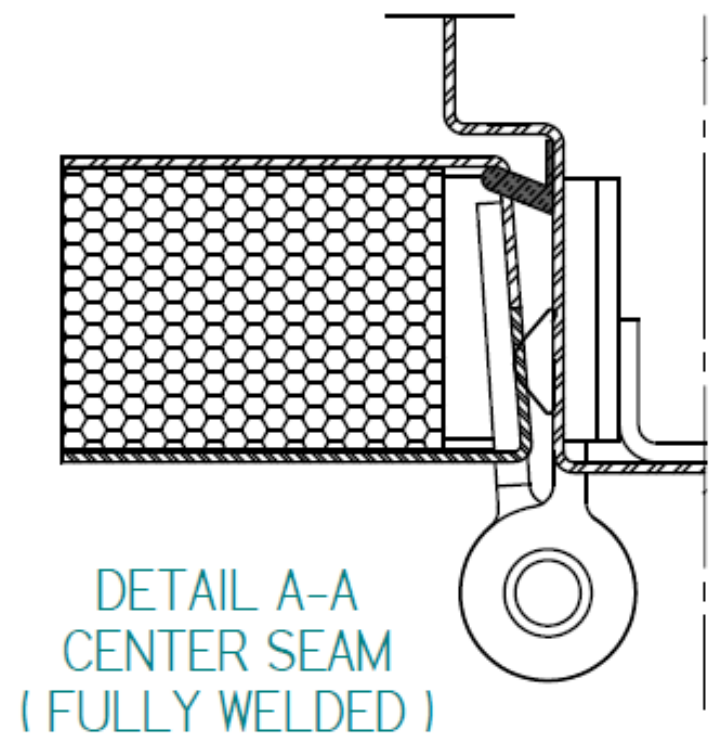

Figure 5.3: Section A-A, fully welded door.

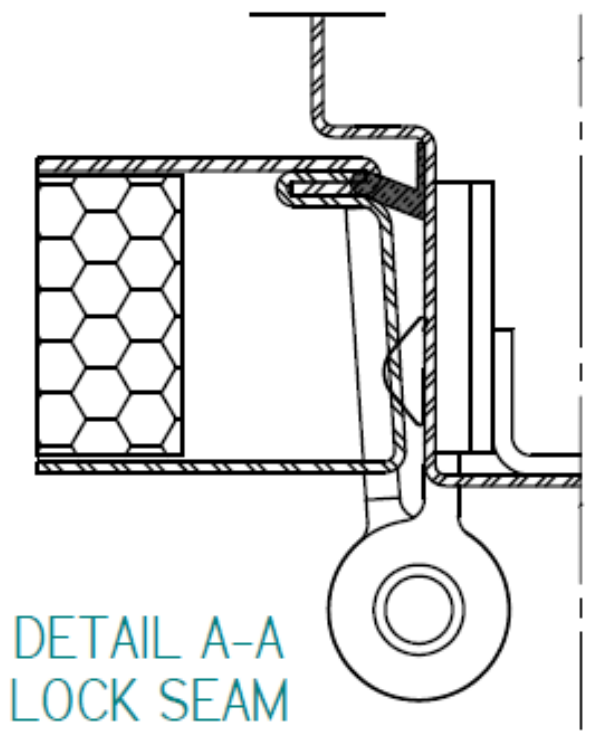

Figure 5.4: Section A-A, lock seam door. 


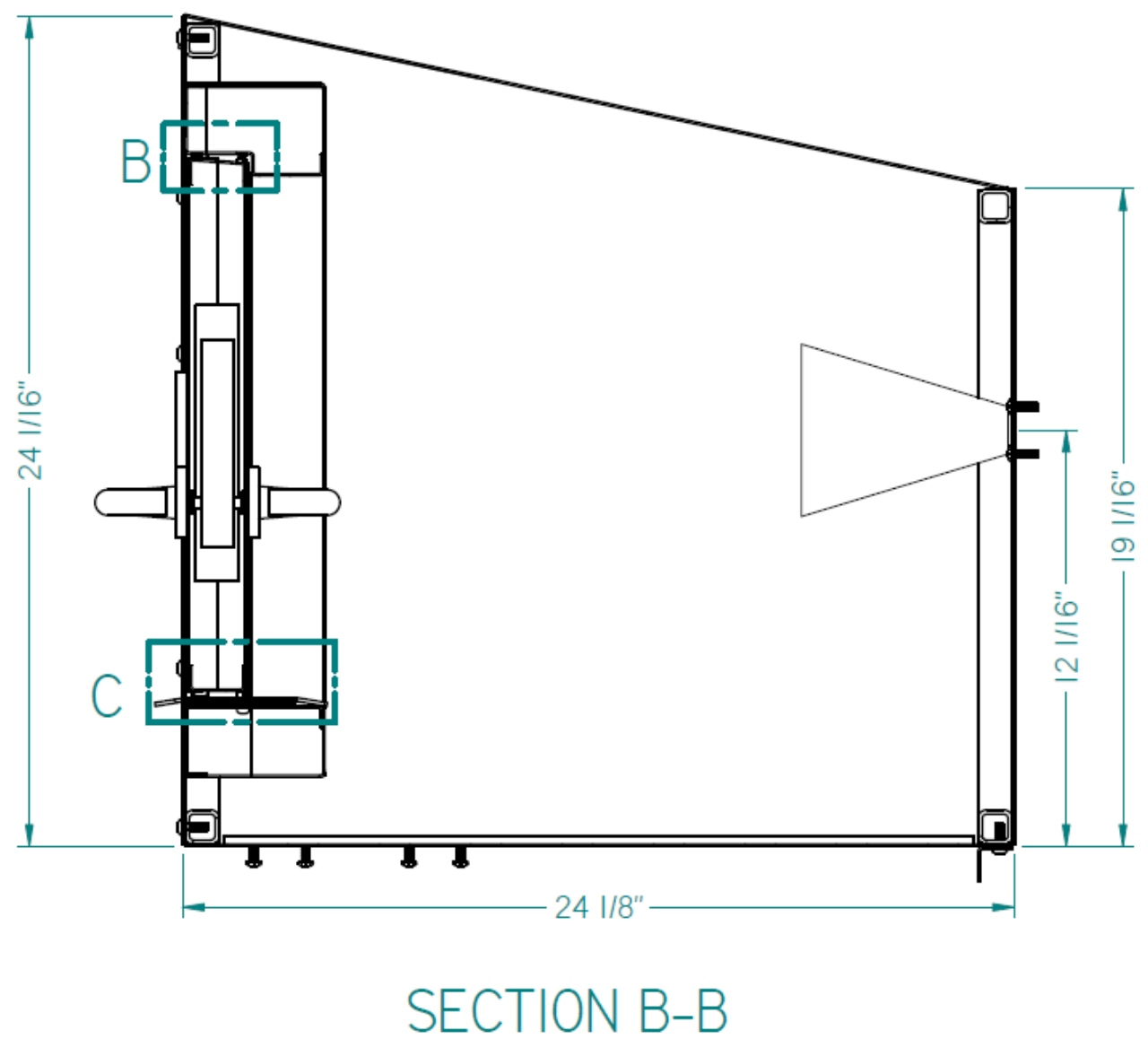

Figure 5.5: Section B-B of Miniaturized frame assembly.

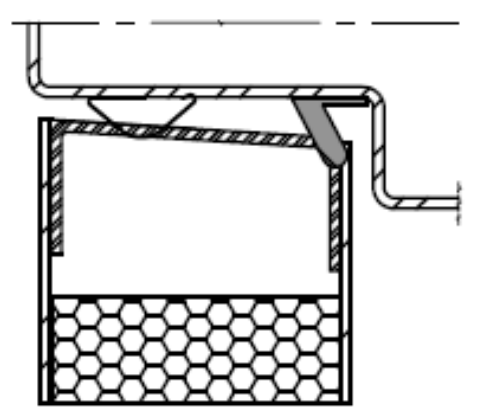

DETAIL B-B

Figure 5.6: Section B-B, top seam.

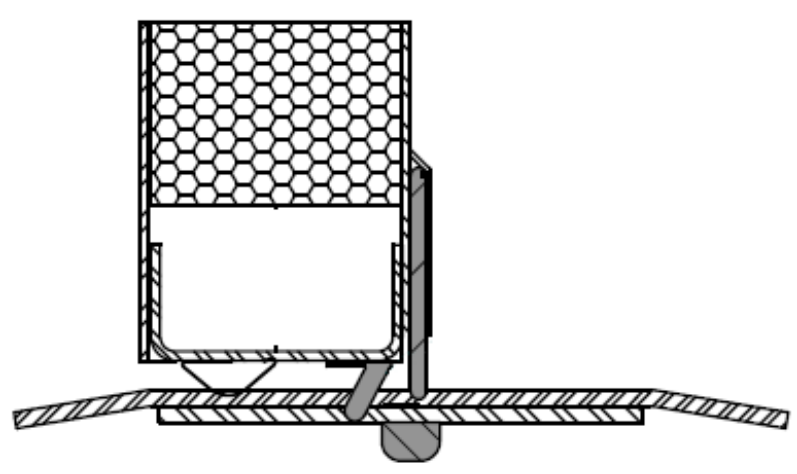

DETAIL C-C

Figure 5.7: Section C-C, bottom seam. 
The final build of the housing is shown below in Fig 5.8 without the door and front panel.

The receiving antenna is a broadband double ridged antenna. It's linearly polarized and highly calibrated for EMI measurements.

The wall panels of the housing were spot welded and reinforced with corner brackets and screws. The copper tape on the edges provides higher shielding effectiveness by ensuring continuous contact between each panel.

To avoid ground reflection as much as possible, the housing was elevated to 24 inches off the ground. The bottom was screwed to a large PVC pipe with two pieces of wood for support at the back.

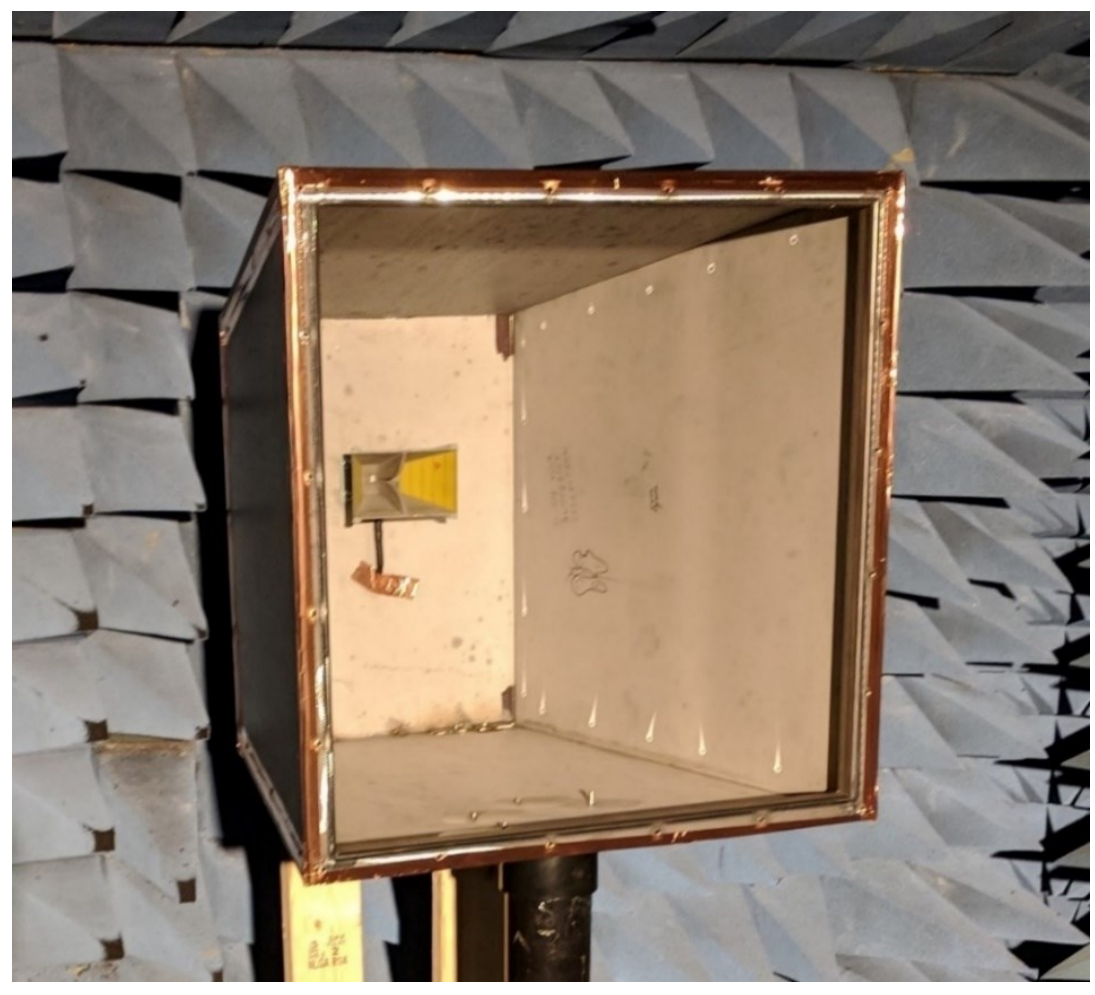

Figure 5.8: Miniaturized assembly frame housing. 


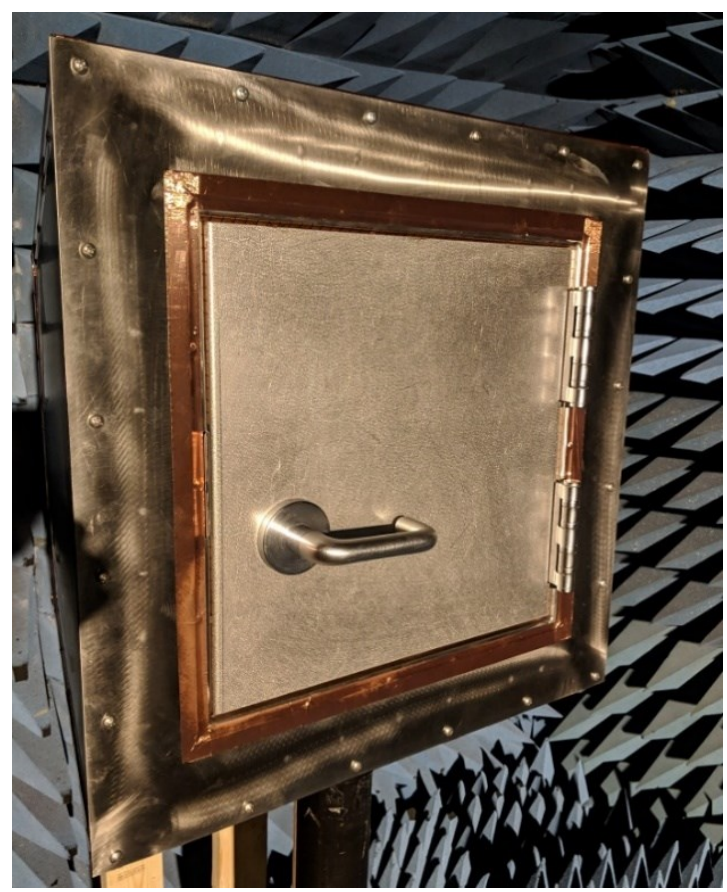

Figure 5.9: Miniaturized door and frame.

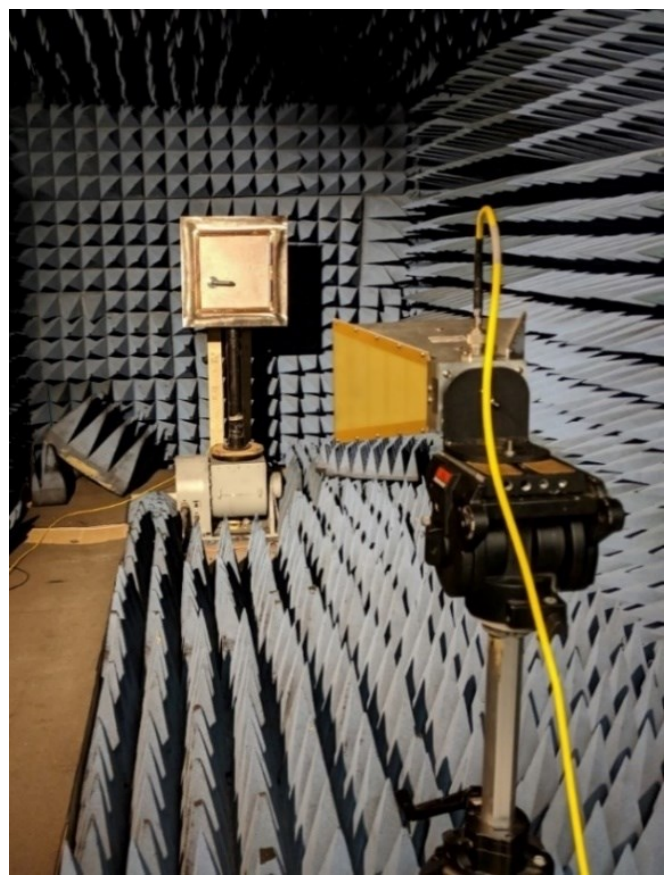

Figure 5.10: SE measurement setup.

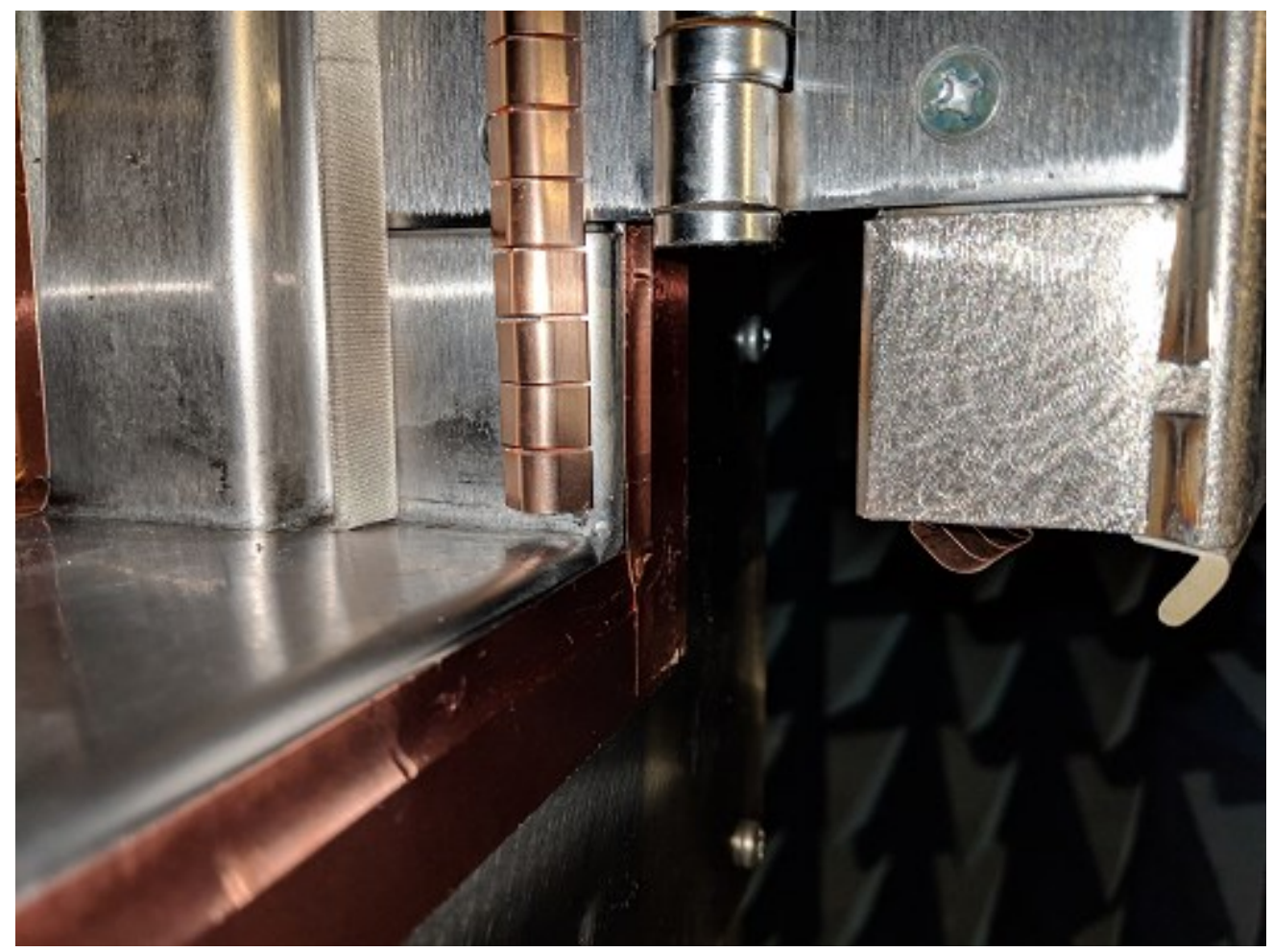

Figure 5.11: Bottom seam and threshold. 
The full set up including the transmit antenna is shown below in Fig 5.10. The transmit antenna is double ridged and broadband like the receiver. It's mounted on a tripod and elevated to the appropriate height to allow the EM waves to be centered at the door.

\subsection{Fully Welded VS Lock Seam}

This test looks at the difference in SE between both door constructions. This is relevant since lock seam edges are less costly and far less time-consuming to fabricate. The results of the SE measurements on both doors are shown in Fig 5.13 and the difference in SE on a log scale is shown in Fig 5.14.

The results show that both doors are comparable in overall SE. The average difference in $\mathrm{SE}$ is $0.67 \mathrm{~dB}$. This indicates that the lock seam design is well suited for the full-size model of the door due to its low cost.

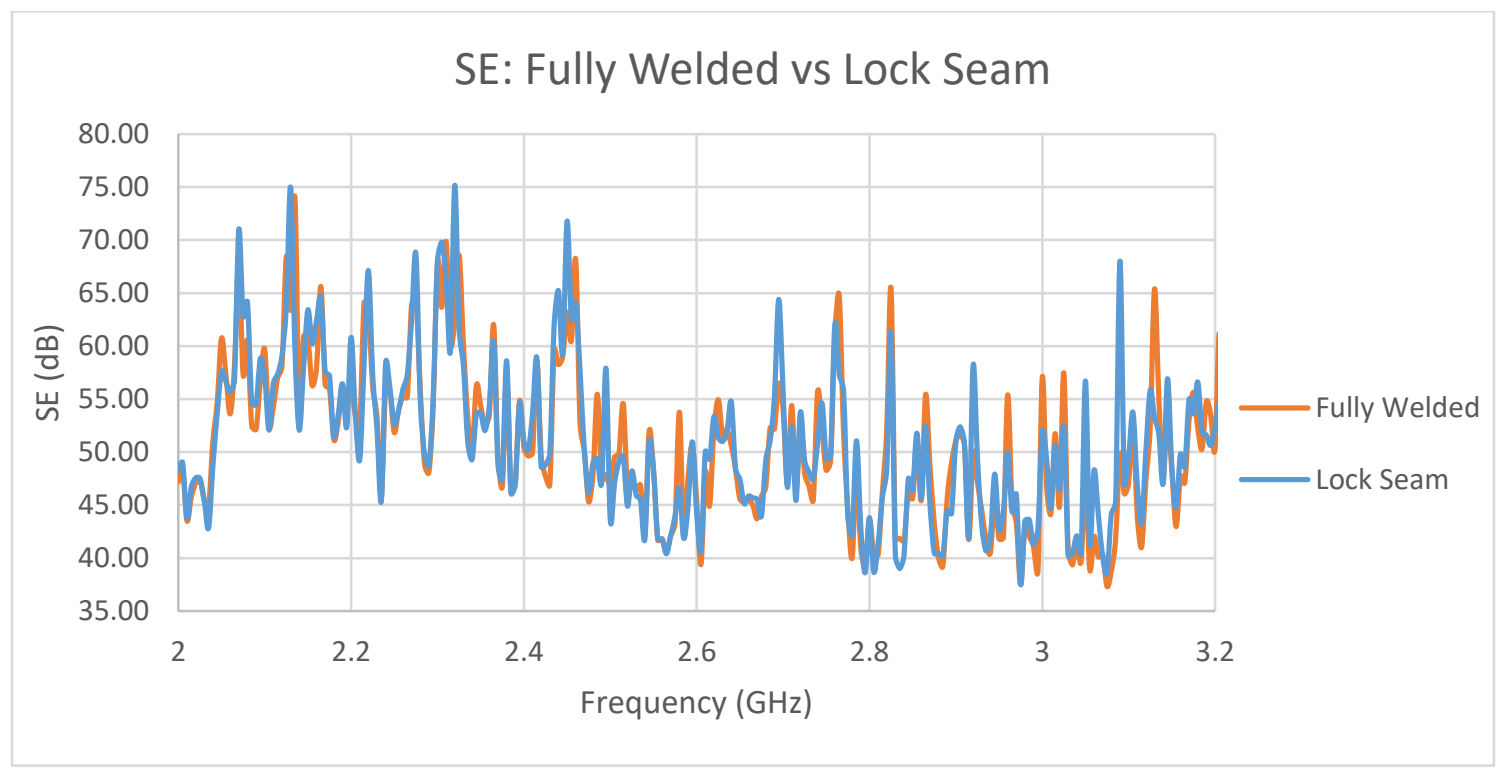

Figure 5.12: SE of fully welded and lock seam door in the anechoic chamber. 


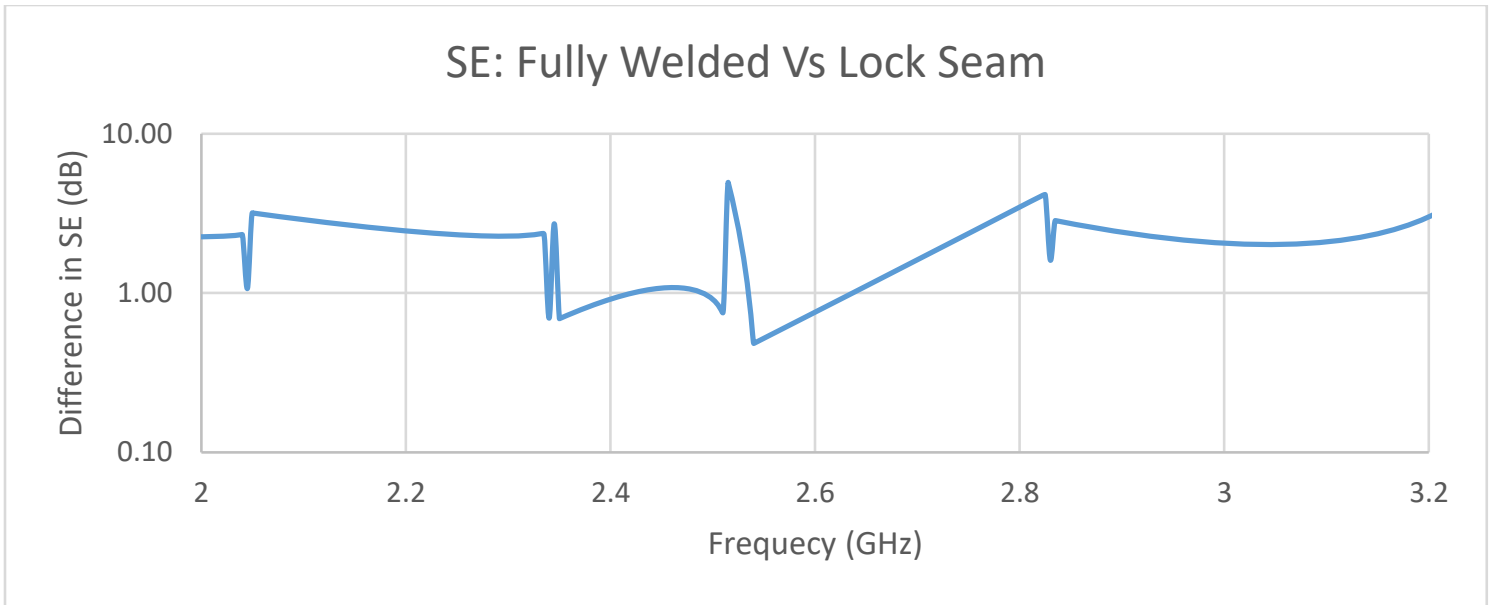

Figure 5.13: Difference in SE between fully welded and lock seam door.

\subsection{Gasket SE}

This test looks at the improvement in SE when both gaskets are used at the same time. The door used in the test had lock seam edges and included the threshold. The initial test was performed with just the c-fold profile FOF gasket. Copper finger stock was later added in front of the FOF. The measurement results are shown below in Fig 5.15.

The results show an improvement in SE when both gaskets are used. The average improvement is $5.5 \mathrm{~dB}$ when finger stock is added to FOF. The difference in SE of both cases is shown in Fig 5.16. 


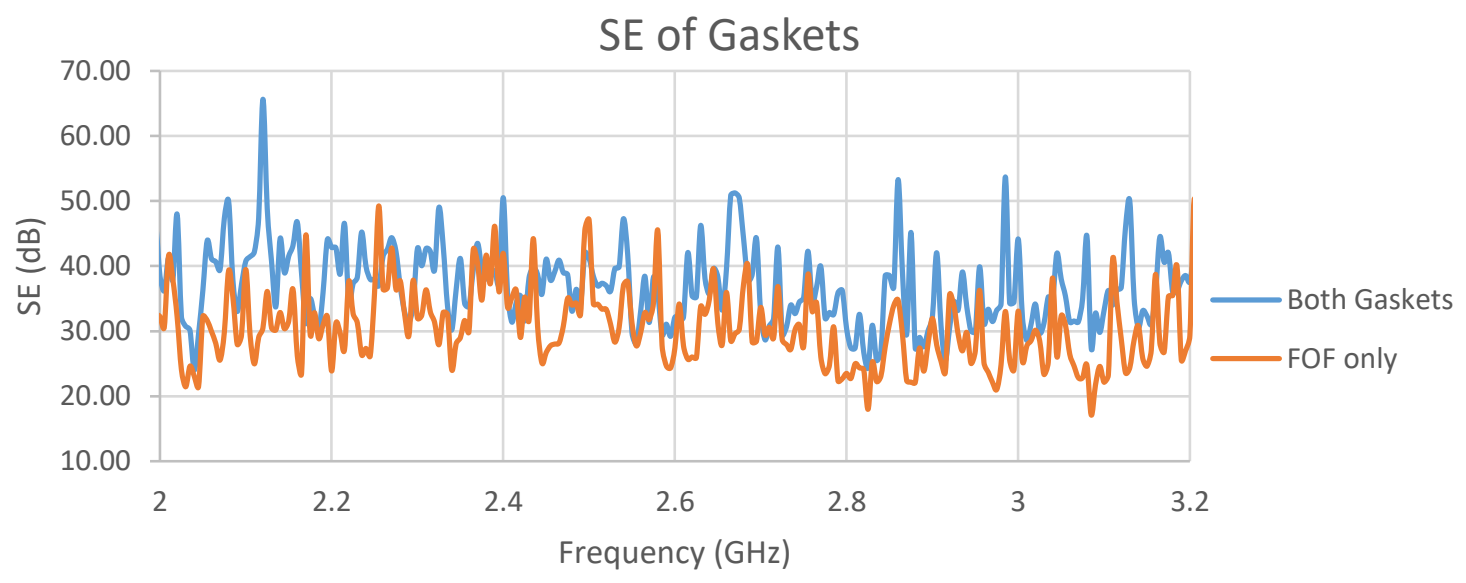

Figure 5.14: Comparison of SE of FOF only and both gaskets.

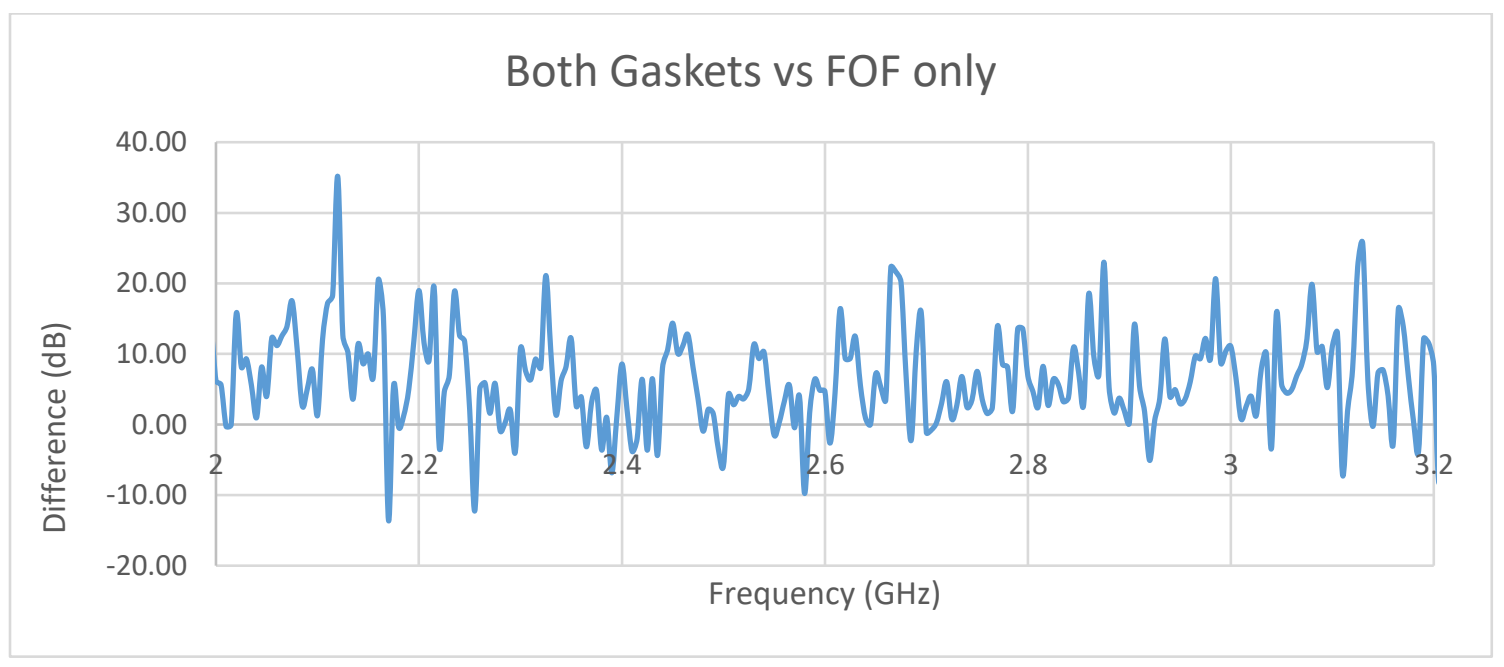

Figure 5.15: Difference in SE between FOF and both gaskets.

\section{$5.4 \quad$ Threshold Leakage}

The two doors used in this test had fully welded edges. The first door had no threshold and used both types of gaskets under the door. The second door had both gaskets including the threshold. The shielding performance of both doors is shown below in Fig 5.17 . 
The measurement results show that the threshold introduces significant leakage when compared to just having a sealed bottom seam between the door and the frame. The average difference in SE between both cases is $12.5 \mathrm{~dB}$. This leakage is the result of the gaps introduced by the threshold. The tolerances in the threshold fabrication left gaps between the threshold and the frame on both sides. There was also a gap created underneath the threshold and the frame due to misalignment when it was screwed into the floor.

The copper tape was used to seal the gaps between the threshold and the frame. Sealing the gaps resulted in an improvement of $5.5 \mathrm{~dB}$ in SE. The measurement results after sealing the gaps are shown below in Fig 5.18.

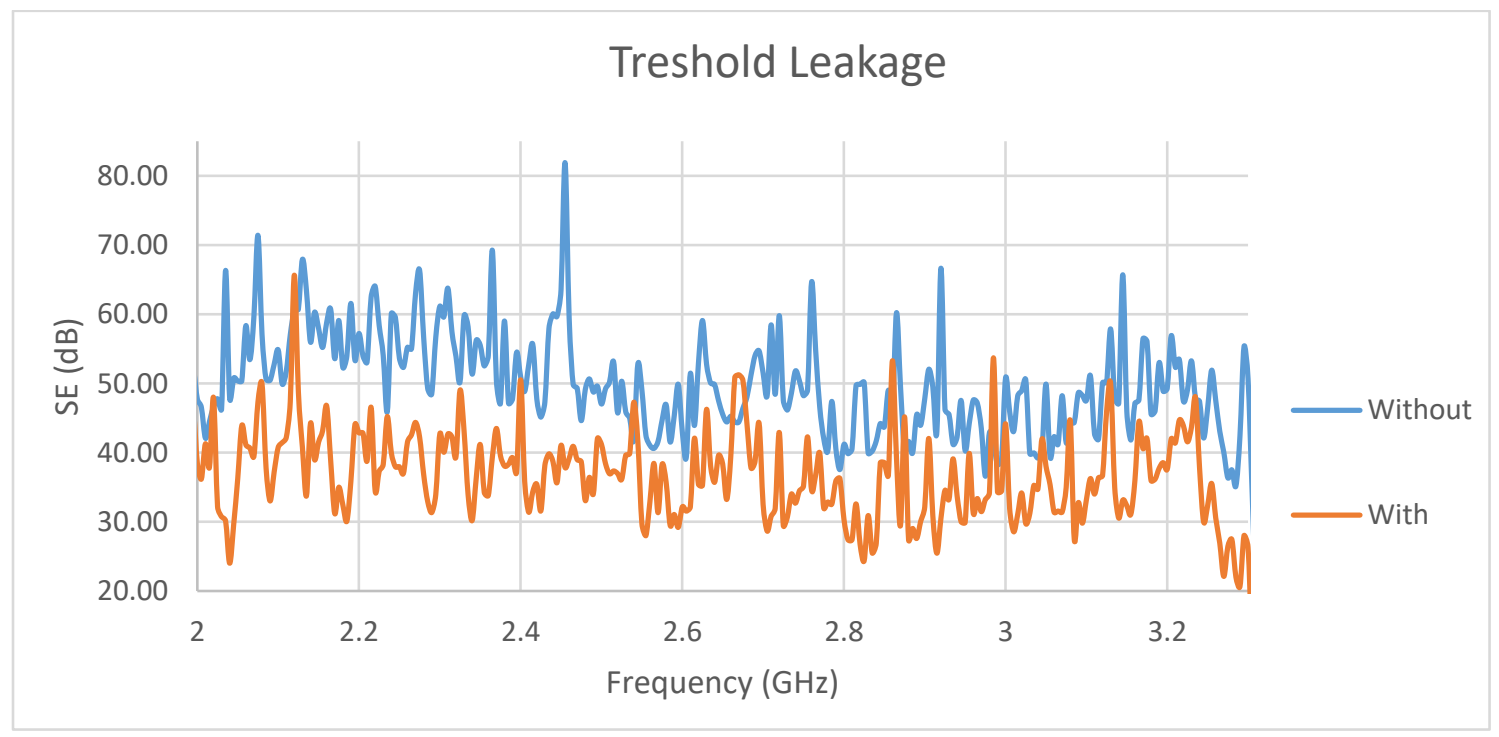

Figure 5.16: Comparison of SE between the door with and without threshold. 


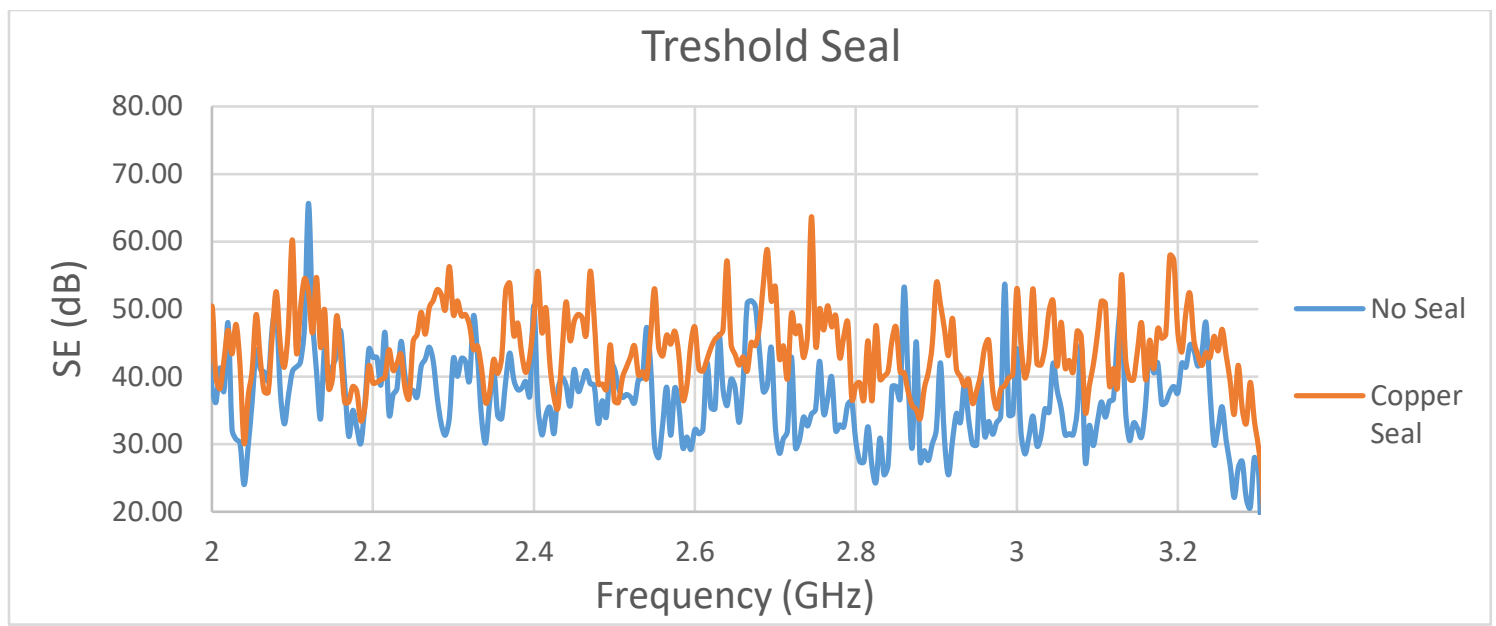

Figure 5.17: Comparison of SE between copper sealed and unsealed threshold.

\subsection{Full-Size SE Testing}

The full-size door and frame assembly were tested for SE at Raymond EMC. The assembly was installed in an enclosure rated for $100 \mathrm{~dB}$ of SE. Several antennas were used in the measurements of SE as shown in Table 5.1. This is necessary since each antenna has a frequency range of operation. The dipole and log periodic antennas overlap in the frequency of operation and were both used to obtain the best measurement at $1 \mathrm{GHz}$.

Table 5.1: List of antennas used in SE measurements.

\begin{tabular}{|l|l|l|l|l|}
\cline { 2 - 5 } \multicolumn{1}{c|}{} & \multicolumn{2}{l|}{ Antenna } & \multicolumn{2}{l|}{} \\
\hline Frequency (MHz) & Loop & Dipole & Log Periodic & Horn \\
\hline 0.01 & $x$ & & & \\
\hline 0.2 & $x$ & & & \\
\hline 1 & $x$ & & & \\
\hline 400 & & $x$ & & \\
\hline
\end{tabular}




\begin{tabular}{|l|l|l|l|l|}
950 & & $x$ & & \\
\hline 1000 & & $x$ & $x$ & \\
\hline 2000 & & $x$ & & \\
\hline 3400 & & & $x$ & \\
\hline 4600 & & & $x$ & \\
\hline 5600 & & & & $x$ \\
\hline 10000 & & & & $x$ \\
\hline 28000 & & & & \\
\hline
\end{tabular}

The measurement locations around the assembly inside and outside the enclosure are shown below in Figure 5.19. Measurements were taken at all eight locations around the door at each frequency in Table 5.1. The lowest measurement of the eight locations at each frequency is the reported measure of SE.

For IP protection the results of the SE measurements will not be shown here. A comparison of the difference in SE between this work and the first prototype is shown in Figure 5.19. The comparison is only for the data points that coincide with both the testing of the prototype and this work. Several more data points were taken for this work as shown in Table 5.1 but cannot be shown here. The design did meet the target goal of a minimum of $50 \mathrm{~dB}$ of SE over the range of $1 \mathrm{GHz}$ to $28 \mathrm{GHz}$. This goal was set by Ambico to compete in the marketplace. 


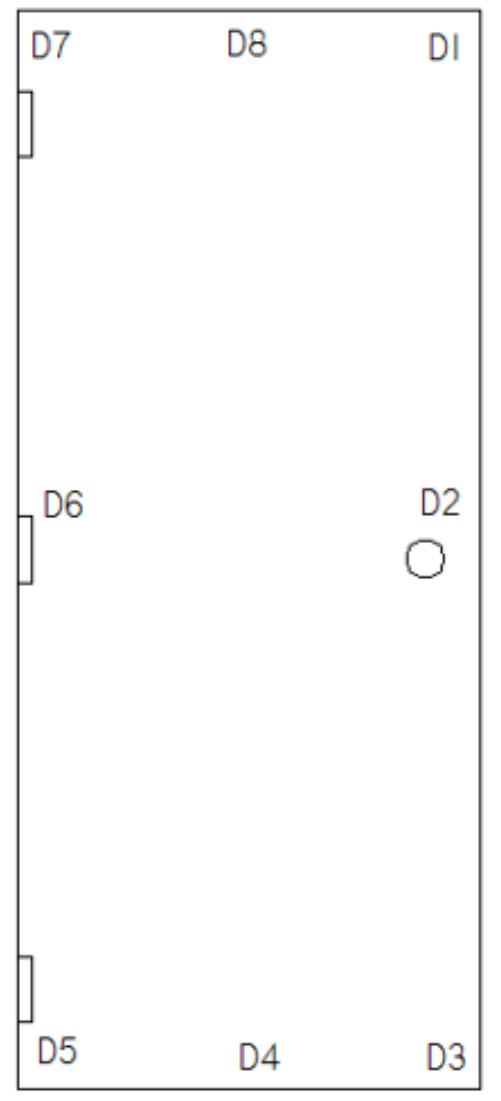

Figure 5.18: SE measurement locations around the door assembly.

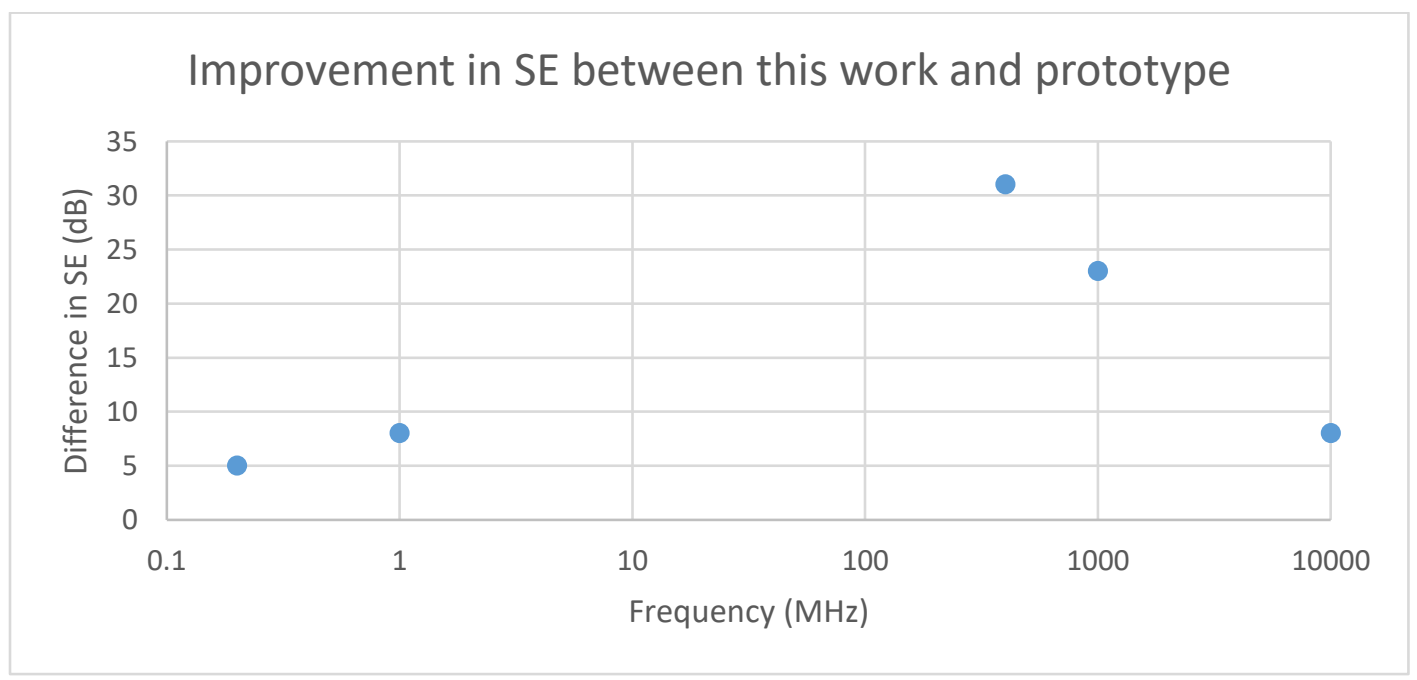

Figure 5.19: Improvement in SE of this work and the prototype. 


\subsection{Conclusion}

SE measurements were performed on a miniaturized version of the door and frame assembly in the Department of Electronics at Carleton University. The measurements were performed in the anechoic chamber. The full-size door and frame assembly measurements were performed at Raymond EMC.

The SE measurement at Carleton shows that there is very little difference in the SE performance between fully welded and lock seam doors. These results can be used to save on the cost of manufacturing since fully welded doors are more costly to fabricate. The measurement results also showed that by using both types of gaskets at the same time, an average improvement of $5.5 \mathrm{~dB}$ was seen compared to just using the FOF gasket. The gaps and misalignment introduced by the threshold had a $12.5 \mathrm{~dB}$ degradation in SE when compared to a shielded bottom alone. By sealing the gaps introduced by the threshold with copper tape, an average improvement in SE of $5.5 \mathrm{~dB}$ was seen in the results. These results point to the need of minimizing the fabrication tolerances of the threshold.

The full-size assembly measurements showed a minimum improvement of $5 \mathrm{~dB}$ in SE when compared to the prototype designed by Ambico. 


\section{Chapter 6}

\section{Conclusions, Contributions, and Future Work}

\subsection{Conclusions}

This thesis has presented the design of an RF shielding door for various applications in the medical and commercial sectors. The possible applications include shielding for MRI and SCIF rooms. The theory presented explains the fundamentals for attenuating electromagnetic energy between two regions in space for shielding purposes. Methods for analysing and measuring the SE of a shielding door have been presented including the use of gaskets to shield seams.

The design process and choices for the materials used in the fabrication of the door in this work are presented. Which includes the choice of gaskets used, specifically $\mathrm{BeCu}$ fingerstock and $\mathrm{NiCu}$ fabric over polyurethane foam. The importance of galvanic compatibility to prevent corrosion when choosing metals for a shielding door design has also been discussed.

Simulations were performed to obtain the SE of the top and side seams of the door and frame assembly in Ansys EDT. The simulations showed that the gasket placement along the depth of the seam changes the frequency response and therefore the SE of the seam. There was a discrepancy between the simulation results for gasket compression compared to theory and industry empirical data. This may have been due to improper gasket 3D model height. Which would result in increased transfer impedance and therefore reduced SE. The SE of a seam can be tailored by using both types of gaskets as 
shown by the simulation results. This in conjunction with gasket placement along the depth of a seam should provide more degrees of freedom in SE control.

The anechoic chamber testing of the miniaturized door assembly at Carleton University showed that there was an insignificant difference in SE between a fully welded and lock seam door. This was significant since the time and cost of fabricating a fully welded door are much higher. When both gasket types were used together to seal the seams compared to FOF alone an improvement of around $5.5 \mathrm{~dB}$ in SE was shown in the measurement results. The tolerances in the threshold fabrication and misalignment with the frame produced an average degradation in SE of around $12.5 \mathrm{~dB}$ compared to a sealed bottom seam with both gaskets. The copper tape was added to seal the gaps around the threshold which resulted in a $5.5 \mathrm{~dB}$ improvement in SE. This showed how critical it is to minimize gaps and misalignment of the threshold during fabrication and installation.

The full-size door and frame assembly testing at Raymond EMC of this work showed an improvement of $5 \mathrm{~dB}$ minimum compared to the measurements made on the earlier prototype. The goal of achieving a minimum SE of $50 \mathrm{~dB}$ was achieved. 


\subsection{Contributions}

The work in this thesis has led to 2 contributions:

1. The use of electromagnetic simulations with $3 \mathrm{D}$ models to obtain relative SE improvements based on gasket configurations at the seams.

2. The use of scaled-down door and frame assembly to obtain the relative SE performance for various gasket and door configurations.

\subsection{Future Work}

Future research in this work should include:

- A mechanism to move the gaskets along the depth of the seams to find the optimum locations that maximises the SE. It could be something like a slide ruler for the gasket locations. This would be useful since the gaskets are stuck on the frame using adhesive. During testing, it becomes tedious to remove a gasket and install a new one since the old one is deformed when it is removed.

- A mechanism for proper gasket alignment around all four sides of the door. This could involve machining tracks on all four sides of the frame to ensure gasket alignment. This would be useful for aligning the gaskets in the corners where it is challenging.

- A corner gasket design to reduce the gap spacing when two fingerstock gaskets meet in the corner. This would reduce the leakage at the higher frequencies. 


\section{References}

[1] C. Paul, Introduction to electromagnetic compatibility, 2nd ed. Hoboken, N.J.: Wiley-Interscience, 2006.

[2] "MRI SHIELDINGARCHITECTURAL SITE PLANNING GUIDE", Mriquestions.com, 2021. [Online].

Available:http://mriquestions.com/uploads/3/4/5/7/34572113/etslindgren_mri_shielding_architechural_planning_guide_2009.pdf. [Accessed: 18Aug- 2020].

[3] A. ABLOY, "How RF Shielding Gives SCIF Rooms Extra Layer of Security", cecodoor.com, 2021. [Online]. Available: https://www.cecodoor.com/en/cecodoor-education/blog-entries/how-rf-shielding-gives-scif-rooms-extra-layer-ofsecurity/. [Accessed: 16- Jan- 2020].

[4] H. Ott, Electromagnetic compatibility engineering. Hoboken, N.J.: Wiley, 2013.

[5] F. Ulaby and U. Ravaioli, Fundamentals of applied electromagnetics, 7th ed. Upper Saddle River: Pearson, 2015.

[6] "GALVANIC COMPATIBILITY", Orbel.com, 2014. [Online]. Available: https://www.orbel.com/sites/orbel.com/files/documents/orbelgalvcompatiblity.pdf. [Accessed: 19- Jul- 2020].

[7] J. Weibler, "Properties of Metals Used for RF Shielding", EMC Test \& Design, vol., 1993. Available:

https://mriq.com/uploads/3/4/5/7/34572113/lindgren_metals_used_for_rf_shi elding.pdf. [Accessed 9 August 2020].

[8] "10 Differences Between Aluminum and Stainless Steel | Metal Supermarkets", Metal Supermarkets.com, 2014. [Online]. Available:

https://www.metalsupermarkets.com/10-differences-aluminum-stainless-steel/. [Accessed: 15- Jul- 2020].

[9] W. Gale, T. Totemeier and C. Smithells, Smithells metals reference book. Oxford [England]: Elsevier Butterworth-Heinemann, 2004, pp. 22-1-22-162. 
[10] "Metal Prices - Steel, Aluminum, Copper, Stainless, Rare Earth, Metal Prices, Forecasting | MetalMiner", 2021. [Online]. Available: https://agmetalminer.com/metal-prices/. [Accessed: 24- Sep- 2020].

[11] "EMI/RFI Shielding | Tech Etch", Tech Etch, 2021. [Online]. Available: https://techetch.com/emi-rfi-shielding/. [Accessed: 17- Jun- 2019].

[12] R. Borgersen, "Choosing the Right EMI Shielding Gasket", Evaluation Engineering, 2000. [Online]. Available:

https://www.evaluationengineering.com/home/article/13001726/choosing-theright-emi-shielding-gasket. [Accessed: 27- Sep- 2019].

[13] "Fingerstock", Euro Technologies, 2021. [Online]. Available: https://www.eurotechnologies.eu/en/fingerstock/. [Accessed: 04- Aug- 2020].

[14] G. Bradlee, "High-Performance Fabric-Over-Foam Gaskets", Evaluation Engineering, 2003. [Online]. Available:

https://www.evaluationengineering.com/home/article/13002187/highperforma nce-fabricoverfoam-gaskets. [Accessed: 23- Jun- 2019].

[15] "Conductive Fabric Over Foam (FOF)", Made-in-China.com, 2021. [Online]. Available: https://semgcapacitor.en.made-inchina.com/product/vXHESjeGrwcn/China-Conductive-Fabric-Over-Foam-FOF.html. [Accessed: 20- Jun- 2020].

[16] "Electrically Conductive Fabric over Foam Gasket Materials", Shielding Solutions Ltd, 2021. [Online]. Available: https://shieldingsolutions.com/product/electrically-conductive-fabric-over-foam-gaskets/. [Accessed: 27- Jan- 2020].

[17] "ASTM D3574 Testing Equipment for Flexible Cellular Urethane Foams", Testresources.net, 2021. [Online]. Available:

https://www.testresources.net/applications/standards/astm/astm-d3574testing-for-slab-bonded-and-molded-flexible-cellular-urethane-foams/. [Accessed: 22- Sep- 2020].

[18] "5 Core Types for Hollow Metal Doors", Beaconcdl.com, 2017. [Online]. Available: http://www.beaconcdl.com/5-core-types-for-hollow-metal-doors/. [Accessed: 14- Jul- 2019]. 